\title{
Joint Cloud and Wireless Networks Operations in Mobile Cloud Computing Environments
}

\author{
by \\ Zhiyuan Yin \\ A thesis submitted to the \\ Faculty of Graduate Studies and Research \\ in partial fulfillment of the requirements for the degree of
}

Master of Applied Science in Electrical and Computer Engineering

Ottawa-Carleton Institute for Electrical and Computer Engineering (OCIECE)

Department of Systems and Computer Engineering

Carleton University

Ottawa, Ontario, Canada, K1S 5B6

January, 2015

(C) Copyright 2015, Zhiyuan Yin 
The undersigned hereby recommends to the

Faculty of Graduate Studies and Research

acceptance of the thesis

\title{
Joint Cloud and Wireless Networks Operations in Mobile Cloud Computing Environments
}

\author{
submitted by \\ Zhiyuan Yin, M.A.Sc \\ in partial fulfillment of the requirements for the degree of \\ Master of Applied Science in Electrical and Computer Engineering
}

Prof. F. Richard Yu, SCE, Carleton, Thesis Supervisor

Chair, Prof. Roshdy H.M. Hafez,

Department of Systems and Computer Engineering

Carleton University

January 2015 


\section{Abstract}

In mobile cloud computing systems, cloud computing has a significant impact on wireless networks. Cloud computing and wireless networks have traditionally been addressed separately in the literature. In this thesis, we jointly study the operations of cloud computing and wireless networks in mobile computing environments, where the objective is to improve the end-to-end performance of cloud mobile media delivered through mobile cloud computing systems. Unlike most existing studies on wireless networks, where only the spectrum efficiency is considered, we consider not only the spectrum efficiency in wireless networks but also the pricing information in the cloud, based on which power allocation and interference management in the wireless networks are performed. We formulate the problems encountered in the operations of mobile cloud computing environments, including determining the price to charge for media services, resource allocation, and interference management, as a Stackelberg game model. Moreover, we extend this game model with multiple players through network virtualization technology, and adopt the replicator dynamics method to solve the evolutionary game between the different groups of small cells. Furthermore, a backward induction method is used to analyze the proposed Stackelberg game. Simulation results are presented to show the effectiveness of the proposed techniques. 


\section{This thesis is the dedication to my family.}

For their endless love, support and encouragement.

A special feeling of gratitude to my beloved grandmother.

I long to hear your voice again, for real not in my dreams.

I am missing you. 


\section{Acknowledgments}

First of all, I would like to express my sincere appreciation to my supervisor, Prof. F. Richard Yu for his steadfast support and wonderful supervision. His technical insight and on-going encouragement have been a constant source of motivation.

I would like to acknowledge Prof. Halim Yanikomeroglu for his advices when I was in the $5 \mathrm{G}$ research team. Besides, I want to thank Prof. Marc St-Hilaire for his unfailingly kind and considerate. It was a really joyful time to be as a TA to work with you. I also would like to thank Prof. Shaobo Ji for his invaluable suggestions to encourage me pursuing my degree.

Many thanks to my colleagues Mr. Zhexiong Wei, Mr. He Zhu, Dr. Yegui Cai, Dr. Hongwei Wang, Dr. Bingyi Guo, Mr. Yanwei Wang, Mr. Chengchao Liang, and the other graduate students and visitors in lab AA509 and OCIECE for their assistance and happy time spent together. Thanks also go to friends in Ottawa, Mr Xiaoning Zhang, Mr. Jingwei Shi and any other individual who generously provides friendship during my years in this city.

Moreover, I greatly appreciate my family and my girl friend Miss Yinlin Zhang for your enduring patient and understanding throughout the courage of my studies. 


\section{Table of Contents}

$\begin{array}{lll}\text { Abstract } & \text { iii }\end{array}$

Acknowledgments $\quad$ v

Table of Contents vi

List of Tables $\quad$ ix

List of Figures $\quad x$

List of Abbreviations $\quad$ xi

1 Introduction 1

1.1 Research Overview ...................... . . 1

1.2 Research Motivations . . . . . . . . . . . . . . . . . 3

1.3 Thesis Contributions . . . . . . . . . . . . . . . . . 4

1.3.1 Accepted and Submitted Papers . . . . . . . . . . 5

1.4 Thesis Organization . . . . . . . . . . . . . . . . 5

2 Background and Related Works $\quad 7$

2.1 Mobile Cloud Computing . . . . . . . . . . . . . . . 7

2.1.1 Features of Mobile Cloud Computing . . . . . . . . . . . . 10

2.1.2 Mobile Cloud Computing Business Service Models . . . . . . . 11 
2.1.3 Network Virtualization . . . . . . . . . . . . . . . . . . 13

2.1.4 Mobile Cloud Service General Model . . . . . . . . . . . . . . 14

2.1.5 Heterogeneous Wireless Networks . . . . . . . . . . . . . . . . 17

2.2 Game Theory . . . . . . . . . . . . . . . . . . . . . . . . . . 19

2.3 Chapter Summary . . . . . . . . . . . . . . . . . . . . 22

3 Cloud and Wireless Network Operations in Mobile Cloud Comput$\begin{array}{lr}\text { ing Environments } & 23\end{array}$

3.1 System Description and Formulation . . . . . . . . . . . . . 23

3.1.1 Telecom Operator Cloud and Third Party CMM SPs . . . . . 24

3.1.2 Heterogeneous Wireless Networks with Small Cells . . . . . . 27

3.1.3 A Game-Theoretic Approach . . . . . . . . . . . . . . . . 28

3.2 Three-Stage Game Analysis . . . . . . . . . . . . . . . . . . . 33

3.2.1 SBS level Game Analysis . . . . . . . . . . . . . . . . . . . 33

3.2 .2 MBS Level Game Analysis . . . . . . . . . . . . . . . 35

3.2 .3 CMM SP Level Game Analysis . . . . . . . . . . . . . . . . 39

3.2.4 Service Allocation Iteration Algorithm . . . . . . . . . . . . . 43

3.3 Simulation Results and Discussions . . . . . . . . . . . . . . . . 45

3.4 Chapter Summary . . . . . . . . . . . . . . . . 53

4 Cloud and Wireless Network Operations through Network Virtual$\begin{array}{ll}\text { ization } & 55\end{array}$

4.1 System and Description . . . . . . . . . . . . . 55

4.1.1 Cloud Platform Model with Network Virtualization Technology 56

4.1.2 Heterogeneous Wireless Networks . . . . . . . . . . . 59

4.1 .3 Problem Formulation . . . . . . . . . . . . . . . . . 61

4.2 Stackelberg Game Analysis . . . . . . . . . . . . . . . . . 66

vii 
4.2.1 SBS Level Game Analysis . . . . . . . . . . . . . . . . . 67

4.2.2 MBS and TOC Level Game Analysis . . . . . . . . . . . . . 68

4.2.3 CMM SPs Level Game Analysis . . . . . . . . . . . . . . . . . 72

4.3 Service Allocation Algorithms . . . . . . . . . . . . . . . . . . . . 75

4.3.1 Evaluative Protocols for SBSs . . . . . . . . . . . . . 76

4.3.2 Protocols for TOC and MBS Group . . . . . . . . . . . 76

4.3.3 CMM Service Allocation Iteration Algorithm . . . . . . . . . . 77

4.4 Simulation Results and Discussions . . . . . . . . . . . . . . . . 81

4.5 Chapter Summary . . . . . . . . . . . . . . . . . . . 83

5 Conclusions and Future Work $\quad 85$

5.1 Conclusions . . . . . . . . . . . . . . . . . . 85

5.2 Future Work . . . . . . . . . . . . . . . . . . . . . 86

$\begin{array}{ll}\text { List of References } & 87\end{array}$ 


\section{List of Tables}

3.1 Notations . . . . . . . . . . . . . . . . . . . . . . . 31

3.2 Simulation Parameters and Assumptions for Performance Evaluations 47 


\section{List of Figures}

2.1 The primary features of mobile cloud computing. . . . . . . . . . 9

2.2 The mobile cloud service general model. . . . . . . . . . . . . . . 16

2.3 The heterogenous wireless networks model. . . . . . . . . . . . . . . . 18

2.4 Four categories of game theory of resources sharing. . . . . . . . . . . 21

3.1 A mobile cloud computing system with telecom operator cloud. . . . 25

3.2 Telecom operator cloud. . . . . . . . . . . . . . . . . 26

3.3 Three-stage Stackelberg game model. . . . . . . . . . . . . . . . . . . 29

3.4 Service allocation with various lowest prices offered by the CMM service providers. . . . . . . . . . . . . . . . . . . . . . . . . . . . 48

3.5 Service allocation in one SBS with various lowest service prices offered by the MBS. . . . . . . . . . . . . . . . . . . . . . . 49

3.6 Service allocation in the MBS with various values of $\alpha$ parameter. . . 49

3.7 Utility function of one SBS. . . . . . . . . . . . . . . . . . 50

3.8 The performance of CMM service in the MBS. . . . . . . . . . . 50

3.9 CMM service iteration step. . . . . . . . . . . . . . . . . . 51

3.10 Network latency hurts interactive performance even with good band-

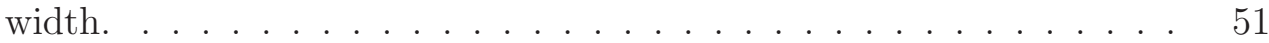

3.11 The average profit versus service allocation on one SBS. . . . . . . . 52

4.1 Mobile cloud computing model with HWNs. . . . . . . . . . . . . 57

4.2 CMM service model. . . . . . . . . . . . . . . . . . . . . . . . 58 
4.3 The MNaaS model. . . . . . . . . . . . . . . . . . . . . . . 60

4.4 Stackelberg game model. . . . . . . . . . . . . . . . . . . . . . . . . 61

4.5 Convergence of the evolution among SBSs to the equilibrium. . . . . 79

4.6 Evolutionary equilibrium with the different SBSs group number and the variable interference price. . . . . . . . . . . . . . . 79

4.7 Evolutionary equilibrium with the different SBSs group number and the variable CMM service size. . . . . . . . . . . . . . . . 80

4.8 Best response for interference prices. . . . . . . . . . . . . . . . . . . 80

4.9 The average profit compares with an existing scheme. . . . . . . . . 81 


\section{List of Abbreviations}

$\begin{array}{ll}\text { AWGN } & \text { Additive White Gaussian Noise } \\ \text { bps } & \text { bits per second } \\ \text { CMM } & \text { Cloud Mobile Media } \\ \text { DSL } & \text { Digital Subscriber Line } \\ \text { HWNs } & \text { Heterogeneous Wireless Networks } \\ \text { IaaS } & \text { Infrastructure-as-a-Service } \\ \text { InP } & \text { Infrastructure Provider } \\ \text { LTE } & \text { Long Term Evolution } \\ \text { MBS } & \text { Macro Base Station } \\ \text { MUs } & \text { Macrocell Users }\end{array}$




\begin{tabular}{|c|c|}
\hline MAaaS & Mobile App-as-a-Service \\
\hline MNaaS & Mobile Network-as-a-Service \\
\hline MSaaS & Mobile Software-as-a-Service \\
\hline MIaaS & Mobile Infrastructure-as-a-Service \\
\hline MTaaS & Mobile Testing-as-a-Service \\
\hline MCaaS & Mobile Community-as-a-Service \\
\hline MMaaS & Mobile Multimedia-as-a-Service \\
\hline $\mathrm{NE}$ & Nash Equilibrium \\
\hline OTT & Over-The-Top \\
\hline OFDMA & Orthogonal Frequency-Division Multiple Access \\
\hline PaaS & Platform-as-a-Service \\
\hline QoS & Quality of Service \\
\hline SBSs & Small Base Stations \\
\hline $\mathrm{SP}$ & Service Provider \\
\hline SaaS & Software-as-a-Service \\
\hline
\end{tabular}


SSU Small Cell Secondary User

SDN Software Defined Networking

TCP Transmission Control Protocol

TOC Telecom Operator Cloud

VNC Virtual Network Computing

WNV Wireless Network Virtualization 


\section{Chapter 1}

\section{Introduction}

\subsection{Research Overview}

Recently, cloud computing has drawn a lot of attention from both academia and industry. It has advantages over traditional computing paradigms, such as avoiding capital investments and operational expenses for end-users. The essential characteristics of cloud computing include on-demand self-service, broadband network access, resource pooling, rapid elasticity, and measured service [1]. Furthermore, with recent advances in mobile communication technologies, mobile devices, and mobile applications, more and more end-users access cloud computing systems via mobile devices, such as smart phones. As such, mobile cloud computing is widely considered as a promising computing paradigm with a huge market. In traditional mobile computing systems, mobile devices usually have limited computing and storage capabilities. In contrast, utilizing the powerful computing and storage resources available in the cloud environments, mobile cloud computing can enable the use of cutting-edge multimedia services. In the cloud, the resources have much higher processing and storage capacities compared to what traditional mobile devices can provide. And thus, the cloud can offer a much richer media experience than current mobile applications. 
There are many promising cloud mobile media (CMM) services based on mobile cloud computing, including media storage and downloading services, audio services, and interactive services (e.g., multi-way video conferencing, advertisements, and mobile multiplayer gaming). CMM services will not only make the end-users enjoy a richer media experience from a mobile device, but a more important aspect is that CMM services will also offer new opportunities for CMM service providers (SPs) and telecom operators to offer end-users rich media services that can be delivered efficiently such that end-users are satisfied and have a good quality of experience. For telecom operators, CMM services will narrow the growing gap between the growth in data usage by end-users and revenue earned by SPs. Despite the potential of CMM services, several research challenges still need to be addressed. These include the following: the availability and accessibility of media services, mobile data integrity, user privacy, energy efficiency, response time, and the quality of service (QoS) over wireless networks. Among these challenges in deploying CMM services, one of the most important is the response time experienced by end-users, which is highly dependent on the quality and speed of the network. Indeed, networking has become a bottleneck, and thus, it has a significant impact on the quality of cloud services. This problem becomes more severe in CMM services due to the scarce network capacity, higher bit error rate, and user mobility in wireless networks. One of the main reasons behind the above problem is that cloud computing and network communications are not jointly designed and optimized. Therefore, when the end-users use CMM services they may suffer long response times and a degraded QoS. In this thesis, we propose an approach based on game theory to consider the joint design of cloud computing and wireless networks. In addition, we introduce the deployment of the telecom operator cloud (TOC) as a promising approach for "interchanging and mixing" the CMM services from different third party CMM SPs. 


\subsection{Research Motivations}

Most current CMM services are offered by over-the-top (OTT) players, which provide easy-to-adopt, on-demand services by exploiting ubiquitous connectivity [2]. OTT SPs can offer tons of CMM services, which contain media storage services, downloading services, and interactive services (e.g., multi-way video conferencing, and cloud gaming) $[3,4]$. However, these OTT players do not provide communication or connectivity services, which are offered by traditional telecom operators. Thus, it is necessary to provide a quantitative approach to jointly consider the following problems in a mobile cloud computing environment: the price to charge end-users for CMM services, resource allocation, and the interference management of wireless networks.

To solve the mentioned problems met in the mobile cloud computing environments, an approach based on game theory is proposed to model the interactions between CMM SPs and the end-users in the heterogeneous wireless networks (HWNs) where the objective is to benefit both parties.

The main target of this research is to design a Stackelberg game approach for modelling CMM SPs and end-users in a mobile cloud computing environment. The proposed approach jointly considers the decision of what to charge end-users for CMM services (called the CMM service price decision), allocation of resources (e.g., wireless capacity) and interference management. More concisely, the objectives of this research are summarized below.

- Design a model that combines the cloud computing environments and HWNs together.

- Present a technique based on game theory that provides quantitative values for the CMM service price decision and the power allocation. 
- Perform simulation experiments to evaluate the proposed approach, and prove the existence of Nash equilibrium of the Stackelberg game method.

\subsection{Thesis Contributions}

In this thesis, we jointly study the operations of cloud computing and wireless networks in a mobile computing environment with a TOC. The objective is to improve CMM service. To the best of our knowledge, the design of joint cloud and wireless networks has not been addressed in previous works. The distinct features of this work are outlined below.

- We consider a mobile cloud computing system with TOC, in which CMM SPs and the size of the CMM service can be dynamically selected. In this way, a telecom operator can strengthen its relationships with end-users and third party CMM service providers by acting as a service and billing aggregator. This can be accomplished using network virtualization technology [1].

- The operations of wireless networks (e.g., resource allocation and interference management) are optimized according to the following: CMM traffic, real-time price provided by the CMM SPs, and the cost associated with the CMM services.

- We formulate the problem of determining the CMM service price decision, resource allocation of wireless network resources, and interference management as a multi-level Stackelberg game. Stackelberg game has been successfully used in cooperative communication networks, and other areas [5]. To analyze the proposed game, we use a backward induction method [6] that can capture the sequential dependencies of the decisions in the stages of the game model. In ad-

dition, we propose an iteration algorithm to obtain the Stackelberg equilibrium solution. 
- Extensive simulations are performed to investigate the performance of the proposed techniques. Based on the simulation results, we verify the convergence of the Stackelberg equilibrium iteration algorithms.

- New challenges arise, which have not been addressed by existing research, when jointly considering the dynamics of cloud, CMM services, and wireless networks. We believe that the research and results that are presented will open a new avenue and motive additional research for considering the operations of cloud and wireless networks in a mobile cloud computing environment.

\subsubsection{Accepted and Submitted Papers}

The following papers have been accepted or submitted:

1. Zhiyuan Yin, F. R. Yu, and Shengrong Bu, "Joint Cloud Computing and Wireless Networks Operations: A Game Theoretic Approach," in Proc. IEEE Globecom'2014, Austin, TX, Dec. 2014

2. Zhiyuan Yin, F. R. Yu, and Shengrong Bu, "Joint Cloud and Wireless Networks Operations in the Mobile Cloud Computing Environment with Telecom Operator Cloud," submitted to IEEE Trans. Wireless Comm., Minor Revision, Mar. 2014.

3. Zhiyuan Yin and F. R. Yu, "Joint Cloud and Virtual Wireless Networks Operations in Mobile Cloud Computing Environments," submitted to IEEE Network Magazine, Oct. 2014 .

\subsection{Thesis Organization}

The rest of the dissertation is organized as below:

- Chapter 2 provides a brief survey of related work, and describes background 
information of the concepts used in this research, which includes mobile cloud computing, HWNs, telecom clouds, and game theory.

- Chapter 3 introduces a system model that jointly considers the CMM SPs and HWNs with small cells. Multiple CMM SPs offer CMM service prices to HWNs. Simulation results have been presented to show that the cloud operations have a significant impact on HWNs. Joint optimization of the operations performed by the cloud and wireless networks is necessary because of the unique dynamics between the cloud, CMM services, and wireless networks. By jointly optimizing the operations of clouds and wireless networks, the proposed scheme can significantly improve the performance of the mobile cloud computing systems.

- Chapter 4 extends the system model (discussed in Chapter 3) with multiple players, which includes CMM SPs, TOC, and HWNs with small cells and network virtualization technology. We formulate the problems of determining the CMM service price, wireless power allocation, and interference management as a three-level Stackelberg game model. Simulation results are presented to show the effectiveness of our proposed schemes.

- Chapter 5 concludes the thesis by summarizing key results, and describing directions for future research. 


\section{Chapter 2}

\section{Background and Related Works}

This chapter provides a survey of literatures related to this dissertation.

\subsection{Mobile Cloud Computing}

Several definitions of mobile cloud computing are available. In [7], the authors define the mobile cloud computing as "a rich mobile computing technology that leverages unified elastic resources of varied clouds and network technologies toward unrestricted functionality, storage, and mobility." It serves a multitude of mobile devices anywhere, anytime through the channel of ethernet of Internet regardless of heterogeneous environments and platforms based on the "pay-as-you-use" principle.

Another definition of mobile cloud computing is described in [8]: "commonly, the term mobile cloud computing means to run an application such as Google's Gmail for Mobile on a remote resource rich server, while the mobile device acts like a thin client connection over to a remote server through wireless network. Some other examples of this type are Facebook's location aware services, Twitter for mobile, mobile weather widgets, etc." The authors also consider mobile devices themselves as resource providers to set up a mobile P2P network. Thus, this approach supports user mobility, and recognizes the potential of mobile clouds to perform collective sensing 
as well.

In [9], the authors describe that "mobile cloud computing often involves three foundations, namely cloud computing, mobile computing and networking, and can be considered as an emerging cloud service model following the trend to extend the cloud to the edge of networks."

In this thesis, we adopt the definition made by [10]: "mobile cloud computing is an emergent mobile cloud paradigm which leverages mobile computing, networking, and cloud computing to study mobile service models, develop mobile cloud infrastructures, platforms, and service applications for mobile clients. Its primary object is to deliver location-aware mobile services with mobility to users based on scalable mobile cloud resources in networks, computers, storages, and mobile devices. Its goal is to deliver them with secure mobile cloud resources, service applications, and data using energyefficient mobile cloud resources in a 'pay-as-you-use' model."

Although some excellent works have been done to study cloud computing and wireless networks [10-13], these two important areas have traditionally been addressed separately in the literatures. However, from the perspective of end-to-end applications [14], both cloud and wireless networks are parts of the entire system. The experience in end-to-end applications (e.g., video and TCP-based applications) indicates that the optimized performance in one segment of the whole system does not guarantee the end-to-end performance $[15,16]$. To ensure the optimal usage of the resources of clouds and wireless networks and enable the scalability of the CMM service users [17], joint cloud and wireless networks operations should be used for each CMM service client [18]. 


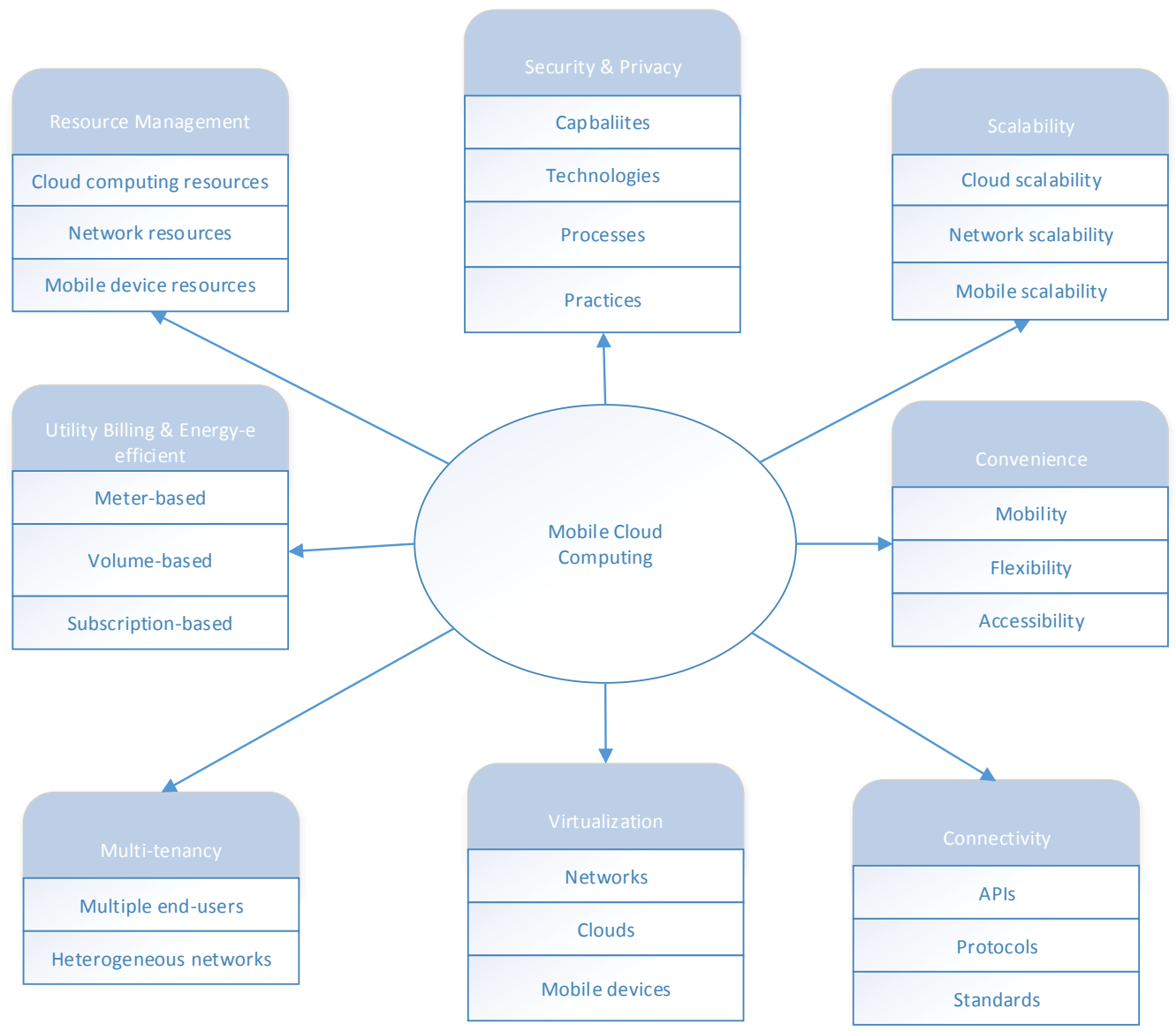

Figure 2.1: The primary features of mobile cloud computing. 


\subsubsection{Features of Mobile Cloud Computing}

The primary features of mobile cloud computing are shown in Fig. 2.1, and are described in detail below.

- Resource Management: mobile clouds can manage the resources more freely and enable resources provisioning and de-provisioning automatically. The resources that need to be managed computing resources, network resources, as well as the resources of mobile devices.

- Security and Privacy: this feature relies on the body of the security capabilities, technologies, processes, and practices. It is designed to protect mobile devices, heterogeneous networks, clouds and the data from attacks, damage, and illegal access.

- Scalability: this feature considers three dimensions for scalability in the mobile cloud computing environments that include the following: cloud scalability, network scalability, and the scalability of the mobile devices.

- Convenience: in the mobile cloud computing environments, the end-users can access the cloud resources (services and applications) at anytime from anywhere.

- Connectivity: this feature adopts the well designed APIs, protocols and standards offered by existing work on mobile clouds to enable easy and secure connectivity between different networks, and third-party applications or systems.

- Virtualization: there are three types of virtualization (network virtualization, cloud virtualization, and virtualization mobile devices) that can be used in the mobile cloud computing environments. 
- Multi-tenancy: this feature allows the mobile cloud to support multiple endusers in heterogeneous networks.

- Utility Billing and Energy-efficient: the mobile cloud provides several service billing models such as meter-based, volume-based and subscription-based.

\subsubsection{Mobile Cloud Computing Business Service Models}

Similar to the cloud computing model, for achieving low-cost media services by using the "pay-as-you-use" approach, mobile cloud computing can also adopt the utility billing model to acquire resources and provide the media services. There are several business service models based on mobile cloud computing as listed below.

- Mobile Software-as-a-Service (MSaaS): the mobile cloud computing can enable Software-as-a-Service (SaaS) and its related functions, which are provided to the end-users with additional features such as mobility, location-awareness, and accessibility from anywhere at anytime. Through this model, the end-users can access the mobile application services deployed on the cloud using the wireless communication technology.

- Mobile Platform-as-a-Service (MPaaS): the mobile cloud computing delivers mobile platforms as a cloud service. It integrates the mobile application management, mobile operator management, and mobile device management built on the cloud to provide maximum scalability and security.

- Mobile Infrastructure-as-a-Service (MIaaS): the cloud infrastructure and its resources are provided to the end-users using "pay-as-you-use" approach. Through MIaaS, all the computing and storage capability of the cloud can be provisioned, manage, and returned according to the demand of the mobile endusers' requests. 
- Mobile Network-as-a-Service (MNaaS): the infrastructure of heterogeneous networks and its related resources are provided by a vendor or "broker" to the mobile end-users in response to on-demand requests. This allows a desirable wireless network infrastructure to be dynamically configured, deployed, and structured for the mobile connectivity to an existing cloud infrastructure. MNaaS provides mobile networking infrastructures as a service [14]. The primary advantage of MNaaS is higher scalability and elasticity. In addition, MNaaS requires a relatively low start-up cost for a network service vendor or "broker". An infrastructure provider or independent telecom operator builds and operates a network (using wireless or transport connectivity) and sells its communication access capabilities to the third parties of clouds charging a price according to capacity utilized by the end-user [19]. An example of MNaaS is OpenStack that is an open-source cloud operating system [20]. It allows end-users to create their own networks, control traffic, and connect servers and devices to one or more networks.

- Mobile App-as-a-Service (MAaaS): it refers to a service business model where diverse mobile applications can be deployed, managed, hosted, and monitored.

- Mobile Testing-as-a-Service (MTaaS): it refers to a service business model where various mobile-based testing devices, tools, and services are provided by a vendor or "brokering" as resources to its mobile clients. Clients use this service to help support the testing of their mobile-based softwares and applications, and are charged using a "pay-as-you-use" or other business models.

- Mobile Community-as-a-Service (MCaaS): it refers to a service business 
model where various mobile social networks and communities can be dynamically established and managed to provide social community services. MCaaS can also be used to provide networking to mobile end-users using a "pay-as-you-use" or other business models.

- Mobile Multimedia-as-a-Service (MMaaS): it refers to a service business model where rich media services based on the application services (e.g., highquality movies and digital games) are deployed, managed, and hosted to deliver the media service to the mobile end-users using a "pay-as-you-use" or other business models.

\subsubsection{Network Virtualization}

Network virtualization is a game-changing technology that enables operators to meet all these evolving demands of its users giving the operators the ability to scale network capacity dynamically and adopt a range of innovative business models.

Virtualization essentially decouples the network hardware from software by introducing a virtual resource manager layer that mediates between the network elements and the software-based network controllers. Software-defined Networking (SDN) is an emerging network architecture which is considered as one of the most promising technologies for realizing virtual networks, especially for managing the control of the network $[21,22]$. The four key features of SDN are summarized below.

- Separation of the control plane from the data plane;

- A centralized controller and view of the network;

- Open interfaces between the devices in the control plane and those in the data plane; 
- Programmability of the network by external applications.

Similar to the wired network virtualization discussed above, wireless network virtualization can have a very broad scope ranging from spectrum sharing, infrastructure virtualization, to air interface virtualization. The physical infrastructures owned by one or more telecom operators can be shared among multiple service providers. Wireless network virtualization needs the physical wireless infrastructure and radio resources to be abstracted and isolated to a number of virtual resources, which can be acquired by different SPs. In other words, virtualization of networks regardless of wired or wireless, can be considered as the process of splitting the entire network infrastructure into virtual resources [23]. However, the distinctive properties of the wireless environments, in terms of time-various channels, attenuation, mobility, broadcast, etc., make wireless network virtualization more complicated.

In this thesis, we define wireless network virtualization (WNV) as the technology in which infrastructure resources and physical radio resources can be abstracted and sliced into virtual wireless network resources that have certain corresponding functionalities, and can be shared by multiple parties through isolation from each other. In other words, virtualizing a wireless network is to realize the process of abstracting, slicing, isolating, and sharing the wireless networks. Above all, it is the key concept of the MNaaS business model. Since wireless network resources are sliced into multiple slices, the terms virtual slice and virtual network have a similar meanings to the term virtual wireless network resources. To avoid confusion, we use the term network virtualization to refer to this entity.

\subsubsection{Mobile Cloud Service General Model}

According to [24], the total revenues of entertainment mobile cloud services (e.g., Amazon's Cloud Drive and Apple's iCloud) are expected to be up to $\$ 39$ billion by 
2016. This situation leads to the explosive growth in mobile data usage, and also means that there will be a dramatic shift regarding how mobility is being used in entertainment areas. The limitations of mobile cloud services are summarized below.

- Limited scalability in network bandwidths and traffic support;

- Limited probability and connectivity between wireless networks that are owned by different telecom operators;

- Limited resource sharing and insufficient usage of network resources;

- Less green computing in networks.

The issues discussed above have become the key challenges of mobile cloud computing. To achieve elasticity and scalability for mobile networking and communication services, the architecture of mobile service system is deployed using following layers as shown in Fig. 2.2:

- Cloud layer: scalable cloud infrastructures and platforms, where diverse backend mobile application servers can be executed, managed, and maintained on the selected cloud for deploying a specific service (e.g., PaaS and SaaS). The objective is to share the computing resources, improve the system utilization, and reduce the cost of the utility billings.

- Mobile cloud layer: this layer consists of the essential services and features critical to mobile cloud computing, such as energy-efficient, provisioning and management of mobile cloud resources, service for mobile application brokering, mobile multimedia services, mobile application services, and mobile cloud security management, etc.

- Network layer: this layer groups, manages, and delivers the network resources 


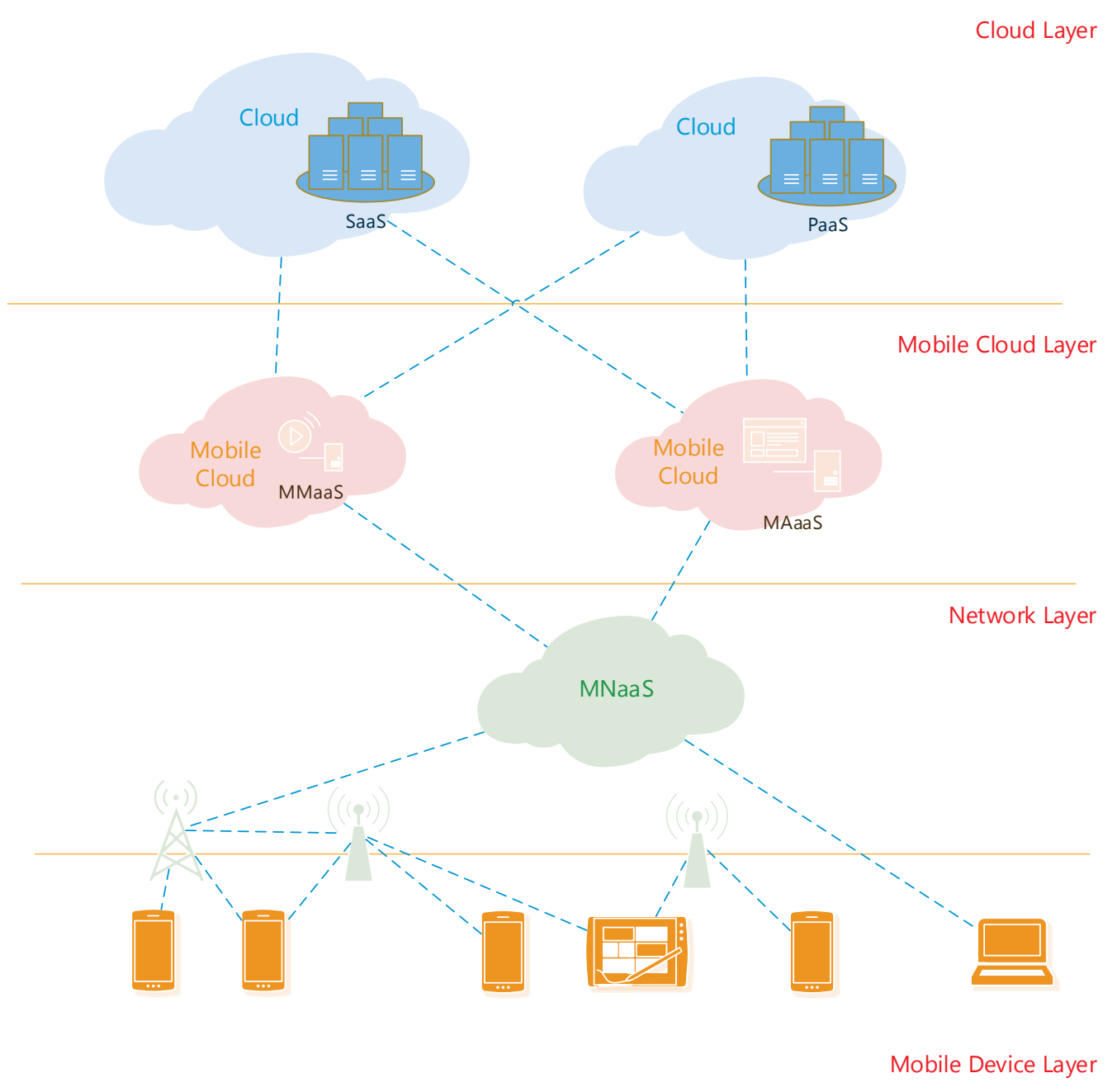

Figure 2.2: The mobile cloud service general model. 
via a MNaaS business model. In addition network virtualization and energysaving techniques are used to achieve the elasticity and scalability of the network resources, which helps achieve higher network resource utilization.

- Mobile device layer: this layer provides the mobile connectivity interfaces to the mobile end-users so that the users can have a consistent and comprehensive access to mobile cloud application services. The essential features of this layer include secure end-to-end mobile transactions and connectivity, as well as maintaining the privacy of end-users.

The mobile service general model offers many advantages as described below.

- The model increases the elasticity and scalability of wireless networks, as well as provide better resource utilization and sharing of resources.

- The model reduces the capital expenditures for the development of diverse mobile network resources, which support a variety of access technologies and combine different market segments.

- The model reduces the cost of operations via use of energy-efficiency techniques and technologies, as well as improves the overall utilization of the network.

- Due to the unified access, this model can reduce the development cost as well.

\subsubsection{Heterogeneous Wireless Networks}

HWNs approach is introduced to solve the "last mile" issue in the mobile cloud computing environments. The deployment of HWNs with small cells is an important technique to increase the energy efficiency of wireless cellular networks [25]. Currently more and more mobile data traffic is being generated by mobile end-users because of activities such as high quality movies, and playing the digital video games. In HWNs, 


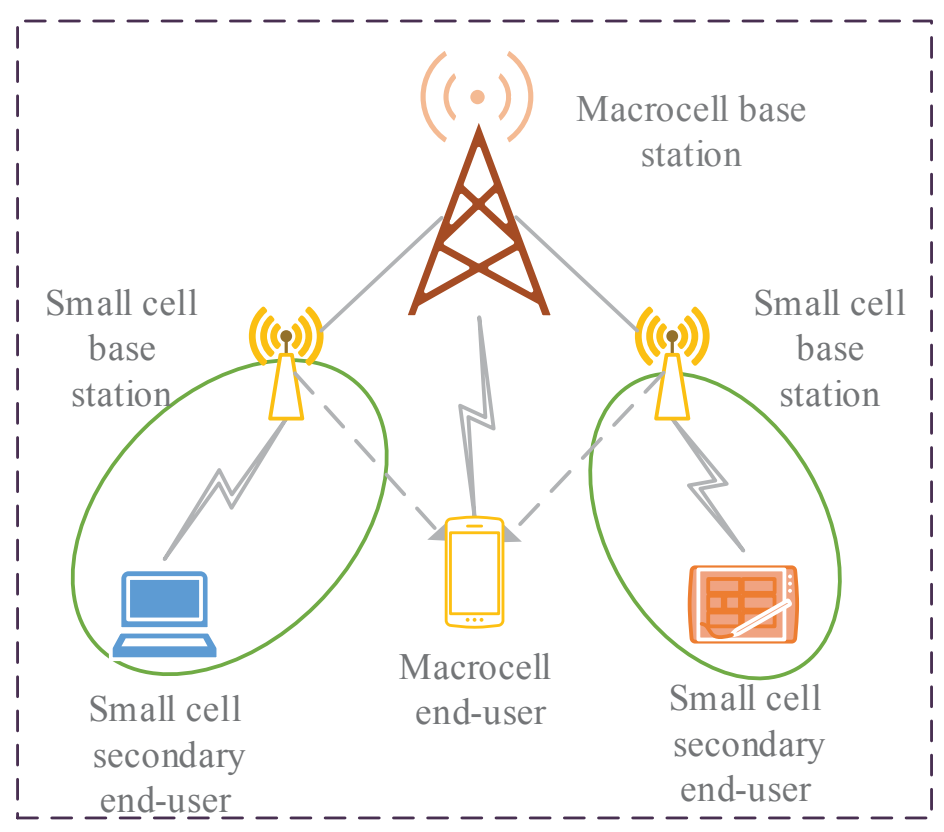

Figure 2.3: The heterogenous wireless networks model.

macrocells have been deployed to provide a larger coverage area, and to improve the mobility of the devices in cellular networks. However, a disadvantage of macrocells is that they cannot deliver high data rates at indoor environments. To solve this issue, small cells are deployed into HWNs to provide higher data rates and to enhance the in-building coverage as well. Due to the coverage area of small cells, they require much less transmission power than the macrocells. Thus by using small cells, base stations become more energy-efficient when providing broad coverage. However, a large number of small cells may cause interference among the macrocells and other small cells, which leads to degradation of performance and energy efficiency of the whole network [26]. Therefore, a deployment of base stations with different cell sizes (macro and small) is desirable in energy-efficient networks. The HWNs model is shown in Fig.2.3. 


\subsection{Game Theory}

Most CMM SPs adopt the "pay-as-you-use" strategy with a fixed CMM service price. They do not consider providing any incentive for end-users to adjust their strategies according to availability or other factors. Therefore, the default agreements offered by CMM SPs always benefit themselves contractually rather than the end-users. To guarantee the benefits for both the CMM SPs and lower the cost for end-users, an approach based on game theory to solve the above problem is introduced [27].

Game theory is a discipline used to model situations in which decision makers have to make specific actions that have mutual, and possibly conflicting interests. It has been used primarily in economics, in order to model competition between companies, and can give an enterprise insights to decide whether or not it should enter a particular market. The first textbook in this area was written by John Von Neumann and Oskar Morgenstern who also were the inventors of game theory [28]. Six years later, John Nash made a number of additional contributions, including describing the Nash equilibrium [29]. In game theory, the Nash equilibrium is a solution concept of a non-cooperative game involving two or more players, in which each player is assumed to know the equilibrium strategies of the other players, and no player has anything to gain by changing only their own strategies [30]. If each player has chosen a strategy, and no player can benefit by changing strategies, while the other players keep their strategies unchanged; then the current set of strategy choices and the corresponding payoffs constitute a Nash equilibrium. This method is more general than the criterion proposed by John Von Neumann and Oskar Morgenstern, since it is applicable to non-zero-sum games, and marks a quantum leap forward in the development of non-cooperative game theory.

Generally speaking, game theory is a mathematical tool, which can analyze the strategic interactions among multiple decision makers [31]. It can be classified into 
four categories in the area of the sharing schemes used in game-theoretic spectrum [32] as shown in Fig. 2.4. The major components in a strategic-form game model are:

- A finite set of players, denoted by $\mathbf{N}$;

- A set of actions, denoted by $A_{i}$, for each player $i$;

- Utility function denoted by $U_{i}: A \rightarrow \mathbb{R}$ that can measure the outcome for player $i$ determined by the actions of all players, $A=\times_{i \in N} A_{i}$.

Given the above definitions and notations, a strategic game is often denoted by $\left\langle N,\left(A_{i}\right),\left(U_{i}\right)\right\rangle$. Since the end-users in HWNs may compete for the limited resources, we can model the interactions between them as a game model, which is detailed as follows.

In the wireless network environments, each end-user makes an intelligent decision on the spectrum usage and operating parameters based on the sensed spectrum dynamics and actions adopted by other end-users. Moreover, end-users who compete for the spectrum resources may have no incentive to cooperate with each other and instead may behave selfishly. Therefore, it is natural to study the intelligent behaviors and interactions of selfish mobile end-users from the perspective of a game-theoretic approach.

The game-theoretic models assume that all the end-users and the CMM SPs are intelligent and rational. In this situation, the end-users pay for the service based on the derived value, rather than the unit they consumed. Secondly, these CMM services are owned by different CMM SPs that can be distributed in different locations. The game-theoretic models are not only decentralized but also offer incentives to the participants, which are suitable to solve the problems in the domain of cloud computing. Hence, the game-theoretic models are appropriate to use in a cloud environment, especially for modelling CMM services. In this dissertation, we choose Stackelberg 


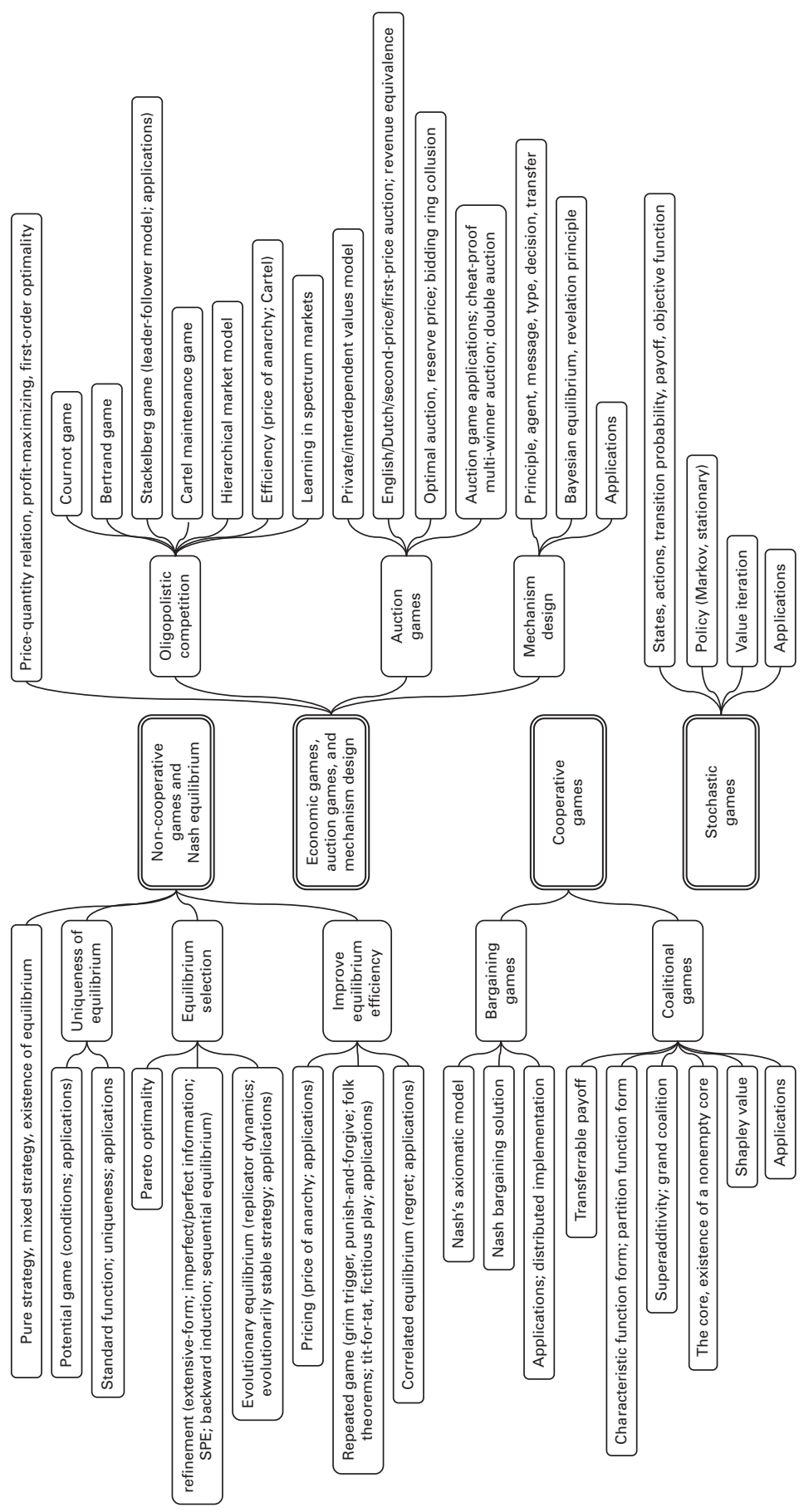

Figure 2.4: Four categories of game theory of resources sharing. 
game model since it has been successfully used in cooperative communication networks, and among other areas. A Stackelberg game is a popular tool to model the situation that consists of a leader and several followers competing with each other for the limited number of resources [5]. Stackelberg game has been successfully used in non-cooperative communication networks [33], and other areas. The leader and followers in a Stackelberg game are usually distributed in space. Similarly, the CMM SPs and end-users are likely to be distributed in space as well.

\subsection{Chapter Summary}

In this chapter, first of all, fundamental concepts concerning mobile cloud computing, game theory are presented. Secondly, the details of the mobile cloud computing features, mobile cloud computing business service models, network virtualization technology, mobile cloud service general model, and heterogeneous wireless networks are discussed. In the next chapters, the proposed game theoretic approaches for cloud and wireless networks operations in the mobile cloud computing environments are presented in detail. 


\section{Chapter 3}

\section{Cloud and Wireless Network Operations in Mobile Cloud Computing Environments}

In this chapter, we firstly describe a mobile cloud computing system including several third party CMM SPs which offer their services to a telecom operator and use the telecom operator as a "broker". Then we introduce HWNs with small cells model to improve the network performance in terms of capacity and energy efficiency. Finally, we formulate the whole systems as a three-stage Stackelberg game and the simulation results are discussed in the end of this chapter.

\subsection{System Description and Formulation}

As shown in Fig. 3.1, we consider a mobile cloud computing system with several third party CMM SPs. The system has a telecom operator cloud that can "mix and interchange" resources offered by different third party CMM SPs, and HWNs contain both macro and small cell base stations. In addition, the problem of joint cloud and wireless networks operations in this system is formulated as a three-stage Stackelberg game. 


\subsubsection{Telecom Operator Cloud and Third Party CMM SPs}

Future media service will definitely be provided by clouds. The multimedia service may come from different cloud service providers, different network types, different technologies, etc. In this chapter, we mainly consider the CMM service model provided by different third party CMM SPs. Different CMM SPs offer rich multi-media services to the end-users, including the streaming media, interactive service, and mobile gaming, etc. In the previous chapter, we have discussed that most of the CMM SPs will choose to partner with the telecom operators rather than to pay. In this scenario, telecom operators will pool variety of third party CMM SPs and offer a virtually unlimited selection of customized and diverse services for end-users.

Telecom operators are making a stronger push to monetize data traffic through OTT-style services and applications. They also attempt to restrict these trafficheavy, low-revenue OTT services such as streaming media, but this may have negative impacts on user experience, even violates the regulations sometimes.

Operators can play a natural role in the cloud computing, providing the reliable and low-cost connectivity service for any other third party clouds, but this is just an initial step. Some of the pioneers in this area have already explored a new TOC model $[1,34,35]$. On one hand, telecom operators can use the powerful storage and computing capabilities offered by the cloud for network management, such as billing, etc. In this case, telecom operators are cloud users. On the other hand, telecom operators can also be cloud providers as well. For example, the telecom operators can leverage the network assets to aggregate and resell the services of third party clouds.

As shown in Fig. 3.2, TOC is in an unique position of being as a cloud "broker" between the wireless networks and the third party SPs, and can manage connectivity and offer flexibility in acquiring network resources on-demand and in real-time.

There are three major roles, namely, cloud connectivity, delivery of cloud-based 

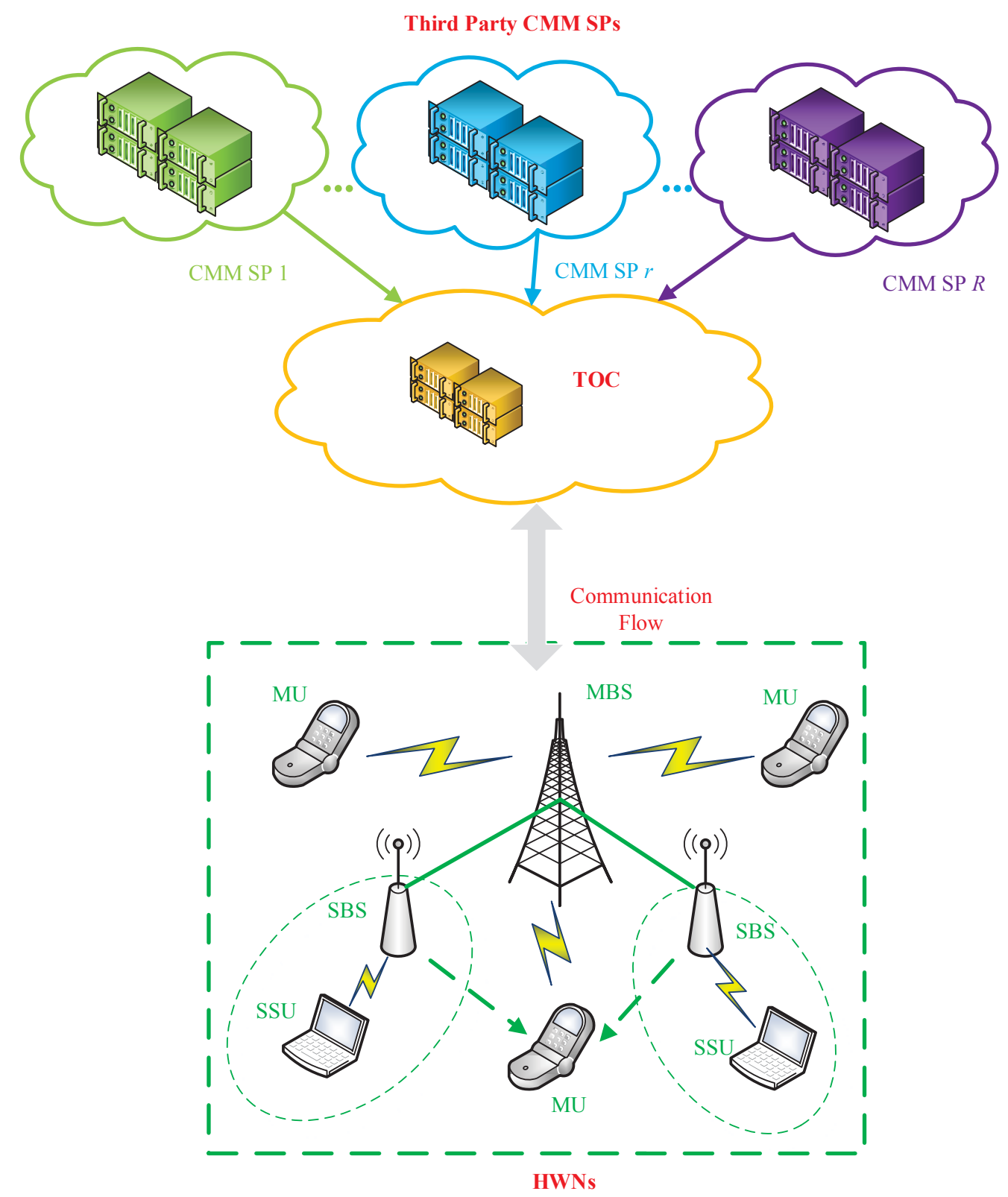

Figure 3.1: A mobile cloud computing system with telecom operator cloud. 


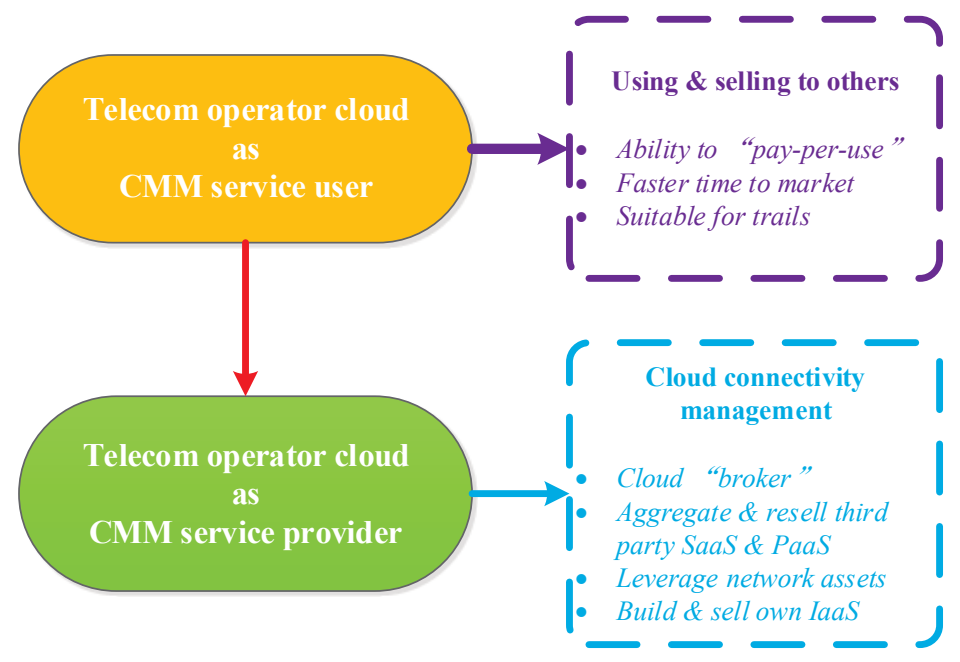

Figure 3.2: Telecom operator cloud.

capabilities, and leveraging network assets to enhance cloud offerings. This TOC model can align itself in the cloud value chain [1].

Pricing is an important issue in cloud computing. There are several studies about pricing schemes and algorithms for cloud services [36-42]. We can group them mainly into two categories, namely static pricing and dynamic pricing. In static pricing schemes, prices cannot be changed in a relatively short period, and the telecom operator cloud does not adapt to real-time congestion conditions, and there is no usage incentives [37-40]. By contrast, dynamic pricing can adjust the prices in nearly realtime in response to the observed network conditions [41,42]. In the mobile cloud environment that is considered in this research, end-users have the abilities to communicate with TOC in real-time. In addition, end-users can easily control their own usage on the individual devices and applications. Therefore, in this chapter, we adopt a dynamic pricing scheme. 


\subsubsection{Heterogeneous Wireless Networks with Small Cells}

A promising approach to improve network performance in terms of capacity and energy efficiency is to use a multi-tier or hierarchical structure with small cells [43]. This architecture represents a novel wireless networking paradigm based on the idea of deploying short-range, low-power, and low-cost base stations, which operate in conjunction with macrocells.

Telecom operators have to deploy multiple wireless access networks with different technologies nowadays to meet the growing demands of users in regards to bandwidth

and mobility [44]. HWNs is one of the solutions to make the handover between these technologies more transparent for the end-users, and to facilitate a more seamless experience for roaming. One of the key features in HWNs is to always provide the best data service and network connectivity to the end-users via different available wireless access networks when subjected to different interworking scenarios appearing throughout the time of handover and roaming procedures.

Fig. 3.1 shows HWNs model, in which there is one macrocell base station (MBS) and multiple small cell base stations (SBSs). Each SBS is connected to the MBS via a broadband connection such as a cable modem or digital subscriber line (DSL). The MBS and the small cells have a cognitive capability and can sense the channel state information. There are multiple macrocell users (MUs) and Orthogonal frequencydivision multiple access (OFDMA) technology is used. To simplify the analysis of this problem, without loss of generality, we assume that each small cell serves only one small cell secondary user (SSU). All the small cells are deploy sparsely to avoid mutual interference. The macrocell and the small cells are considered to be perfectly synchronized and the whole system is operated in a time-slotted manner.

As we consider that the macrocell and the small cell share the spectrum in the 
mobile network, there will be cross-tier interference between them, which will significantly affect the performance. To guarantee the QoS of end-users and reduce this effect, we introduce an interference price charged by MBS to protect itself from the SSU. According to this price and the channel condition, the small cell will change the sub-bands they access and their transmission power.

\subsubsection{A Game-Theoretic Approach}

In this chapter, the problem of joint operations of telecom operator cloud and heterogeneous wireless network is formulated as a three-stage Stackelberg game, which is shown in Fig. 3.3. The main notations used in this chapter are listed in Table 3.1. Each third party CMM SP is a leader that provides a cloud media service price $x_{r}$ to the macrocell and small cells. All the MUs and the SSUs, which are playing the part of followers, decide the amount of media service from the CMM SPs to purchase according to the service price $x_{r}$ in Stage I of the game. We measure the media service in bits per second (bps) to meet the end-user's media demand by guaranteeing performance. In Stage II, firstly, the MBS decides as a follower from which CMM SP to buy the media service, then it acts as a leader to offer an interference price $y$ to the small cells to reduce the interference effect. In the last stage, each SBS decides which CMM SP to buy the service from based upon the service price $x_{r}$ and the interference price $y$ charged by MBS.

Cloud Level Game For the CMM SPs, we assume that each of them is selfish and independent of gaining the revenue as much as possible. Each CMM SP's profit depends on its own resource cost and the service price, as well as the price offered by the other SPs. For an arbitrary provider, we can formulate the utility function $U_{r}(x)$ 


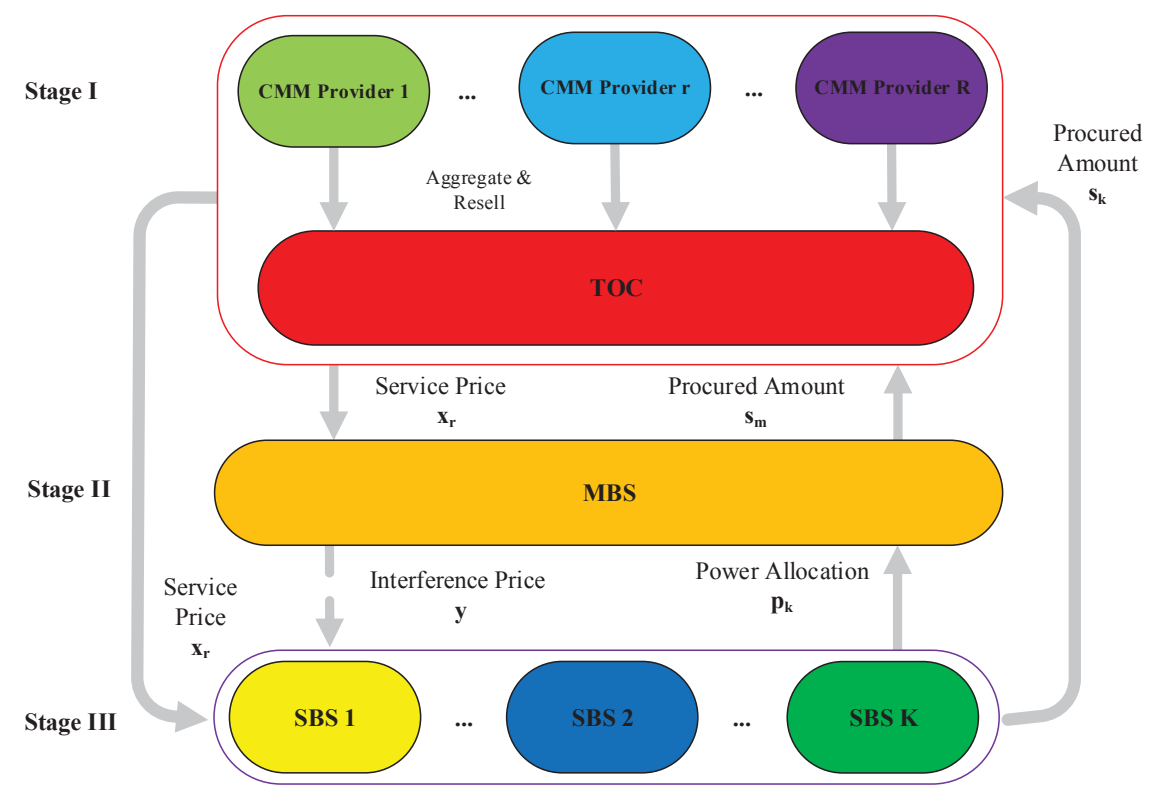

Figure 3.3: Three-stage Stackelberg game model.

below,

$$
U_{r}(x)=\left(x_{r}-c_{r}\right)\left(s_{m} B_{r m}+\sum_{k=1}^{K} s_{k} B_{r k}\right) .
$$

The price vector $\mathbf{x}\left(x=\left\{x_{1}, \ldots, x_{r}, \ldots, x_{R}\right\}\right)$ denotes the prices offered by the SPs, and $c_{r}$ denotes the cost of the CMM SP (e.g., the server cost, infrastructure cost, power usage, and the networking cost) [45]. The set $\boldsymbol{R}(R=\{1, \ldots, \mathrm{r}, \ldots, \mathrm{R}\})$ denotes the number of the game players - CMM SPs. We assume that the cost of each CMM SP is different from the others. $s_{m}$ and $s_{k}$ denote MBS $m$ and SBS $k$ purchase the amount of CMM service from the CMM SP $r . B_{r m} \in\{0,1\}$ and $B_{r k} \in\{0,1\}$ denote whether MBS $m$ and SBS $k$ purchase the service from the SP $r$ or not, where 1 means yes and 0 means no. The SP needs to find an optimal price to the MBS and SBSs in order to maximize its own revenue, which can be solved by 
the following problem:

$$
\max _{x_{r} \geq c_{r}} U_{r}(x)
$$

MBS Level Game and SBS Level Game We need to consider both the transmission data rate and the computing resource consumption. To these ends, the MBS needs to limit the interference from small cells by offering the interference price $y$. We have MBS's net utility function defined below,

$$
\begin{aligned}
& U_{m}\left(s_{m}, p_{m}, y\right) \\
= & \min \left(W \log _{2}\left(1+\frac{p_{m} h_{m}^{2}}{\sigma_{m}^{2}+\sum_{k=1}^{K} g_{k m}^{2} p_{k}}\right), s_{m}\right)-\alpha x_{r} s_{m} B_{r m}+\beta y \sum_{k=1}^{K} g_{k m}^{2} p_{k} .
\end{aligned}
$$

$W$ denotes the transmission bandwidth of each channel, $h_{m}$ denotes the channel gain from MBS $m$ to its scheduled macrocell user including the path-loss and the small-scale fading process, $p_{m}$ denotes the transmit power of MBS $m$ to its scheduled MU, here we assume this power is fixed. $\sigma_{m}$ is AWGN with zero mean and unit variation, and $g_{k m}$ denotes the channel gain between the small cell $k$ and the scheduled MU served by MBS $m$ including the path-loss and the small-scale fading process, and $y$ denotes the interference price. The set $\boldsymbol{K}=\{1, \ldots, k, \ldots, K\}$ and $\boldsymbol{M}=$ $\{1, \ldots, m, \ldots, M\}$ denote the number of the SBSs and MBS in HWNs.

The min operator means that we should consider the smaller value of either demand or supply. We assume that we do not know the size of the capacity of MBS $m$ and the amount of CMM service requested from the end-user scheduled by MBS $m$. If the size of the requirements is greater than the capacity of the MBS $m$, we have to choose the capacity to adapt the real environment, and vice versa. 
Table 3.1: Notations

\begin{tabular}{|c|c|}
\hline Notation & Description \\
\hline$x_{r}$ & Cloud media service price \\
\hline$c_{r}$ & Cost of CMM SP $r$ \\
\hline$y$ & Interference price \\
\hline$s_{m}$ & Amount of CMM service (MBS purchased from CMM SP $r$ ) \\
\hline$s_{k}$ & Amount of CMM service (SBS purchased from CMM SP $r$ ) \\
\hline$B_{r m}$ & Whether MBS $m$ purchases service from provider $r$ \\
\hline$B_{r k}$ & Whether SBS $k$ purchases service from provider $r$ \\
\hline$W$ & Transmission bandwidth of each channel \\
\hline$h_{m}$ & Channel gain between MBS and MU $m$ \\
\hline$h_{k}$ & Channel gain between SBS $k$ and SSU $k$ \\
\hline$p_{m}$ & Transmit power of MBS to MU $m$ \\
\hline$p_{k}$ & Transmit power of SBS $k$ \\
\hline$\sigma_{m}$ & AWGN with zero mean and unit variation in Stage II \\
\hline$\sigma_{k}$ & AWGN with zero mean and unit variation in Stage III \\
\hline$g_{k m}$ & Channel gain between the small cell $k$ and the MU $m$ \\
\hline$\alpha$ & $\begin{array}{c}\text { Parameter of tradeoff between service price } \\
\text { and transmission rate in Stage II }\end{array}$ \\
\hline$\beta$ & $\begin{array}{c}\text { Parameter of tradeoff between the transmission rate } \\
\text { and interference price in Stage II }\end{array}$ \\
\hline$\mu_{k}$ & $\begin{array}{c}\text { Parameter of tradeoff between service price } \\
\text { and transmission rate in Stage III }\end{array}$ \\
\hline$\lambda_{k}$ & $\begin{array}{l}\text { Parameter of tradeoff between the transmission rate } \\
\text { and interference price in Stage III }\end{array}$ \\
\hline
\end{tabular}


$\alpha$ and $\beta$ denote weights, which represent the tradeoff among the transmission rate, service cost and interference revenue. Moreover, to make all the sub-formulas keep the operator symbols unchanged, we assume that $\alpha$ and $\beta$ are greater than 0 . The optimization problem for the MBS can be formulated as,

$$
\max _{s_{m}>0, y \geq 0} U_{m}\left(s_{m}, y\right)
$$

The net utility function for an arbitrary SBS $k(k \in\{1 \ldots K\})$ can be defined below,

$$
U_{k}\left(s_{k}, p_{k}\right)=\min \left(W \log _{2}\left(1+\frac{p_{k} h_{k}^{2}}{\sigma_{k}^{2}}\right), s_{k}\right)-\mu_{k}\left(x_{r} s_{k} B_{r k}\right)-\lambda_{k} y g_{k m}^{2} p_{k} .
$$

The symbol $h_{k}$ denotes the channel gain between SBS $k$ and SSU $k$, including the path-loss and the small-scale fading process, $p_{k}$ denotes the transmit power of SBS $k$, and $\sigma_{k}$ is AWGN. $\mu_{k}$ and $\lambda_{k}$ denote weights, which represent the tradeoff among the transmission rate, service cost and interference cost. Moreover, $\mu_{k}$ and $\lambda_{k}$ are greater than 0 .

The min operator means that we should consider the smaller value of either demand or supply. We assume that we do not know the size of the capacity of SBS $k$ and the amount of CMM service requested from the end-user scheduled by SBS $k$. If the size of the requirements is greater than the capacity of the SBS $k$, we have to choose the capacity to adapt the real environment, and vice versa.

The optimization problem for an arbitrary small cell $k$ can be formulated as,

$$
\max _{s_{k}>0, p_{k} \geq 0} U_{k}\left(s_{k}, p_{k}\right) .
$$




\subsection{Three-Stage Game Analysis}

In this section, we analyze the proposed three-stage Stackelberg game. Then we obtain the equilibrium to this game. Based on the description of the system, we know that each strategy will affect the others. Hence, we will use a backward induction method to solve this game. And an equilibrium point can always be obtained from the argument: starting from the final nodes, each player chooses a best reply given the (already determined) choices of all the players that move after him. This results in an equilibrium point also in each sub-game, whether that sub-game is reached or not. Such a point is called sub-game-perfect equilibrium, or backward induction equilibrium.

\subsubsection{SBS level Game Analysis}

For maximizing the utility function of SBSs, each SBS will choose a proper CMM SP to purchase the CMM service according to the service price $x_{r}$, and the interference price $y$ charged by MBS. For an arbitrary SBS $k$, its utility function will be defined

in two cases: (1) $W \log _{2}\left(1+\frac{p_{k} h_{k}^{2}}{\sigma_{k}^{2}}\right) \leq s_{k} ;(2) W \log _{2}\left(1+\frac{p_{k} h_{k}^{2}}{\sigma_{k}^{2}}\right)>s_{k}$. The first case is the most common condition in real mobile cloud computing environment. In the most cases, the transmission rate of the CMM SP is larger than the transmission rate in the sub-band.

For the first case, we can obtain the utility function as:

$$
U_{k}\left(s_{k}, p_{k}\right)=W \log _{2}\left(1+\frac{p_{k} h_{k}^{2}}{\sigma_{k}^{2}}\right)-\mu_{k} x_{r} s_{k} B_{r k}-\lambda_{k} y g_{k m}^{2} p_{k}
$$

When $1-\mu_{k} x_{r} B_{r k}>0$, in order to maximize $U_{k}$, we can use the decomposition theory. We decompose the optimization problem into two sub optimization problems, fix $s_{k}$ then make $p_{k}^{*}$ to be optimum to obtain the optimal $s_{k}^{*}$. So, its utility function is a 
concave function of $p_{k}$ based on the definition (3.7), since

$$
\frac{\partial^{2} U_{k}}{\partial p_{k}^{2}}=-\frac{W h_{k}^{4}\left(1-\mu_{k} x_{r} B_{r k}\right)}{\ln 2\left(p_{k} h_{k}^{2}+\sigma_{k}^{2}\right)}<0
$$

Therefore, we can obtain the optimal power allocation strategy $p_{k}^{*}$ below:

$$
p_{k}^{*}=\left[\frac{W\left(1-\mu_{k} x_{r} B_{r k}\right)}{\ln 2 \lambda_{k} y g_{k m}^{2}}-\frac{\sigma_{k}^{2}}{h_{k}^{2}}\right]^{+} .
$$

Because the function of $s_{k}$ is monotonic decreasing, then we know that it will achieve the maximum when $s_{k}$ takes the minimum, that is,

$$
\begin{aligned}
s_{k}^{*} & =W \log _{2}\left(1+\frac{p_{k}^{*} h_{k}^{2}}{\sigma_{k}^{2}}\right) \\
& =\left[W \log _{2}\left(\frac{h_{k}^{2} W\left(1-\mu_{k} x_{r} B_{r k}\right)}{\ln 2 \lambda_{k} y g_{k m}^{2} \sigma_{k}^{2}}\right)\right]^{+} .
\end{aligned}
$$

When $1-\mu_{k} x_{r} B_{r k}<0$, the utility function of $p_{k}$ is a convex function, then we can obtain the minimum of the function as below,

$$
p_{k}=\left[\frac{W\left(1-\mu_{k} x_{r} B_{r k}\right)}{\ln 2 \lambda_{k} y g_{k m}^{2}}-\frac{\sigma_{k}^{2}}{h_{k}^{2}}\right]^{+}
$$

Hence, $s_{k}$ can achieve optimal when $p_{k}=0$. The utility function can be a monotonic decreasing function with $s_{k}: U_{k}\left(s_{k}, p_{k}\right)=-\mu_{k} x_{r} s_{k} B_{r k}$. So when $s_{k}^{*}=0$, the function will get the maximum value, but this situation makes no sense in the real environment. Since an SSU applies for service from a mobile provider, its transmission rate should retain above zero.

For the second case, we can obtain the utility function of $s_{k}$ as,

$$
\begin{aligned}
U_{k}\left(s_{k}\right) & =s_{k}-\mu_{k}\left(x_{r} s_{k} B_{r k}\right)-\lambda_{k} y g_{k m}^{2} p_{k} \\
& =\left(1-\mu_{k} x_{r} B_{r k}\right) s_{k}-\lambda_{k} y g_{k m}^{2} p_{k} .
\end{aligned}
$$


When $1-\mu_{k} x_{r} B_{r k}>0, U_{k}\left(s_{k}\right)$ is a monotonic increasing function. To obtain the

maximum value from this function, we know that $s_{k}^{*}=\left[W \log _{2}\left(1+\frac{p_{k}^{*} h_{k}^{2}}{\sigma_{k}^{2}}\right)\right]^{+}$. In here, we will keep the transmission power $p_{k}$ unchanged to make $s_{k}$ achieve the optimal value.

When $1-\mu_{k} x_{r} B_{r k}<0, U_{k}\left(s_{k}\right)$ is a monotonic decreasing function. So when $s_{k}^{*}=0$, the function will get the maximum value, but this situation makes no sense in the real environment as well due to the reasons described above.

\subsubsection{MBS Level Game Analysis}

The MBS, in order to maximize its utility function, will firstly as a follower choose a proper CMM SP to purchase the CMM service based on the service price. Then it is as a leader to offer an interference price to the SBSs. We will obtain $U_{m}$, a function of transmission rate $s_{m}$ and interference price $y$. In here, we consider the most common situation and also use the decomposition method to solve this problem. Firstly we keep the interference price $y$ unchanged to get the optimal $s_{m}^{*}$ to maximize $U_{m}$, then we obtain the desirable value of $y$. We assume that,

$$
\begin{aligned}
I_{m} & =\sigma_{m}^{2}+\sum_{k=1}^{K} g_{k m}^{2} p_{k}^{*} \\
& =\sigma_{m}^{2}+\sum_{k=1}^{K} g_{k m}^{2}\left[\frac{W\left(1-\mu_{k} x_{r} B_{r k}\right)}{\ln 2 \lambda_{k} y g_{k m}^{2}}-\frac{\sigma_{k}^{2}}{h_{k}^{2}}\right]^{+} .
\end{aligned}
$$

In the MBS level, it will choose the CMM SP with the lowest service price $x_{r}^{*}$. For MBS $m$, its utility function will be defined in two cases: (1) $W \log _{2}\left(1+\frac{p_{m} h_{m}^{2}}{\sigma_{m}^{2}+\sum_{k=1}^{K} g_{k m}^{2} p_{k}}\right) \leq s_{m} ;(2) W \log _{2}\left(1+\frac{p_{m} h_{m}^{2}}{\sigma_{m}^{2}+\sum_{k=1}^{K} g_{k m}^{2} p_{k}}\right)>s_{m}$.

For the first case, this situation is the most common condition in real mobile cloud computing environment. In most scenarios, the transmission rate of the CMM SP is larger than the sub-band transmission rate. 
For the second case, it is a rare scene, but we cannot neglect it.

We obtain the utility function in the first case,

$$
U_{m}\left(s_{m}, y\right)=W \log _{2}\left(1+\frac{p_{m} h_{m}^{2}}{I_{m}}\right)-\alpha x_{r}^{*} s_{m}+\beta y \sum_{k=1}^{K} g_{k m}^{2} p_{k}^{*}
$$

Because $\alpha, x_{r} \geqslant 0$, the utility function is monotonic decreasing of $s_{m}$, when $s_{m}$ chooses the minimum value, the function will achieve the maximum value. Then we can obtain the optimum of

$$
s_{m}^{*}=\left[W \log _{2}\left(1+\frac{p_{m} h_{m}^{2}}{\sigma_{m}^{2}+\sum_{k=1}^{K} g_{k m}^{2}\left(\frac{W\left(1-\mu_{k} x_{r} B_{r k}\right)}{\ln 2 \lambda_{k} y g_{k m}^{2}}-\frac{\sigma_{k}^{2}}{h_{k}^{2}}\right)}\right)\right]^{+}
$$

And the utility function in the second condition is shown below,

$$
U_{m}\left(s_{m}, y\right)=s_{m}\left(1-\alpha x_{r}{ }^{*}\right)+\beta y \sum_{k=1}^{K} g_{k m}^{2} p_{k}
$$

When $1-\alpha x_{r}{ }^{*}>0$, to maximize the function, the value of $s_{m}$ will be

$$
s_{m}^{*}=\left[W \log _{2}\left(1+\frac{p_{m} h_{m}^{2}}{\sigma_{m}^{2}+\sum_{k=1}^{K} g_{k m}^{2} p_{k} *}\right)\right]^{+} .
$$

When $1-\alpha x_{r}{ }^{*} \leqslant 0$, it is a monotonic decreasing function. To obtain the maximum of the function, $s_{m}=0$, but this situation makes no sense in the real environment. Since an MU applies for service from a mobile provider, its transmission rate should retain above zero. And we have discussed that only in the condition of $1-\mu_{k} x_{r} B_{r k}>0$, parameter $p_{k}^{*}$ can get the optimal value. Hence, we will continue the steps in the above default condition. 
Due to the piece nature of interference price $y$, we present an indicator function,

$$
D_{k}= \begin{cases}1, & y<\frac{h_{k}^{2} W\left(1-\mu_{k} x_{r} B_{r k}\right)}{\ln 2 \lambda_{k} \sigma_{k}^{2} g_{k m}^{2}}, \forall k \\ 0, & \text { otherwise. }\end{cases}
$$

We can rewrite $U_{m}$ which is shown as (3.19).

$$
\begin{aligned}
U_{m}(y)= & \left(1-\alpha x_{r}^{*}\right) W \log _{2}\left(1+\frac{p_{m} h_{m}^{2}}{\sigma_{m}^{2}+\sum_{k=1}^{K} g_{k m}^{2} D_{k}\left(\frac{W\left(1-\mu_{k} x_{r} B_{r k}\right)}{\ln 2 \lambda_{k} y g_{k m}^{2}}-\frac{\sigma_{k}^{2}}{h_{k}^{2}}\right)}\right) \\
& +\beta y \sum_{k=1}^{K} g_{k m}^{2} D_{k}\left(\frac{W\left(1-\mu_{k} x_{r} B_{r k}\right)}{\ln 2 \lambda_{k} y g_{k m}^{2}}-\frac{\sigma_{k}^{2}}{h_{k}^{2}}\right) .
\end{aligned}
$$

Because $U_{m}$ is a piecewise function of $y$, we cannot solve it by derivation directly. When the value of $D_{k}$ is given, we can obtain the function $U_{m}$ as a continuous differentiable function. We let $N_{k}$ as,

$$
N_{k}=\frac{h_{k}^{2} W\left(1-\mu_{k} x_{r} B_{r k}\right)}{\ln 2 \lambda_{k} \sigma_{k}^{2} g_{k m}^{2}}, \forall k
$$

After having sorted all $N_{k}$ in ascending order, like $N_{1} \leqslant N_{2} \leqslant \ldots \leqslant N_{k}$. Hence, we get $K$ intervals $\left[0, N_{1}\right),\left(N_{2}, N_{3}\right), \ldots,\left(N_{k-1}, N_{k}\right)$. And by piecewise differentiating of function $U_{m}$ in each interval, we can easily obtain it is concave except most $N$ non-differentiable points by analogizing as below,

$$
\frac{\partial U_{m}(y)}{\partial y}=\left(1-\alpha x_{r}^{*}\right) \sum_{k=1}^{K} \frac{W^{2} p_{m} h_{m}^{2}\left(1-\mu_{k} x_{r} B_{r k}\right)}{(\ln 2)^{2} y^{2} I_{m}(y)\left(p_{m} h_{m}^{2}+I_{m}(y)\right) \lambda_{k}}-\beta \sum_{k=1}^{K} g_{k m}^{2} \frac{\sigma_{k}^{2}}{h_{k}^{2}}
$$

Based on (3.21), we know $\frac{\partial^{2} U_{m}(y)}{\partial y^{2}}$ is shown as (3.22). 


$$
\begin{aligned}
\frac{\partial^{2} U_{m}(y)}{\partial y^{2}}= & -\left(1-\alpha x_{r}^{*}\right) \sum_{k=1}^{K} \frac{W^{2} p_{m} h_{m}^{2}\left(1-\mu_{k} x_{r} B_{r k}\right)}{(\ln 2)^{2} \lambda_{k} y^{3}\left(p_{m} h_{m}^{2} I_{m}+I_{m}^{2}\right)^{2}} \\
& \left(p_{m} h_{m}^{2} I_{m}+\left(p_{m} h_{m}^{2}+2 I_{m}\right)\left(\sigma_{m}^{2}-\sum_{k=1}^{K} g_{k m}^{2} \frac{\sigma_{k}^{2}}{h_{k}^{2}}\right)\right)<0 .
\end{aligned}
$$

Therefore, $U_{m}(y)$ is a concave function. And this noncooperative interference price game is a concave game. According to the formulation (3.19), this game exists at least one Nash equilibrium, which can be obtained as follows.

Then, from (3.22), we can know that $\frac{\partial U_{m}(y)}{\partial y}$ is a strictly monotonic decreasing function of $y$.

Obviously, we find that,

$$
\lim _{y \rightarrow \infty} I_{m}=\sigma_{m}^{2}-\sum_{k=1}^{K} g_{k m}^{2} \frac{\sigma_{k}^{2}}{h_{k}^{2}}>0,
$$

then,

$$
\lim _{y \rightarrow \infty} \frac{\partial U_{m}(y)}{\partial y}=-\beta \sum_{k=1}^{K} g_{k m}^{2} \frac{\sigma_{k}^{2}}{h_{k}^{2}}<0 .
$$

For the case of $y \rightarrow 0$ we can obtain that,

$$
\lim _{y \rightarrow 0} \frac{\partial U_{m}(y)}{\partial y}=\infty>0
$$

Therefore, the utility function $U_{m}$ is firstly increasing with the interference price $y$, then at the certain point begins to decrease with $y$. So this utility function is a concave function without some non-differentiable points $N_{k}, k \in(1, \ldots, K)$. That is, this non-cooperative competitive game exists at least one Nash equilibrium. We 
can find this optimal value of $y$ in each interval by multiple methods (e.g., a binary search algorithm and a gradient based algorithm).

\subsubsection{CMM SP Level Game Analysis}

Bertrand game is a popular tool to model competition among firms (sellers) that set prices and their customers (buyers) that choose quantities at the price set. Bertrand game has been successfully applied in cognitive radio networks, and other areas [31]. In our scheme, we use Bertrand game to model the competition among CMM SPs. We assume that each CMM SP is independent, acts selfishly, and the target is to gain as much revenue as possible. If the CMM SPs act noncooperatively, it will lead a monopoly situation. All the CMM SPs are eager to set their service prices as the same, and try to maximize their whole profits. The profit of an arbitrary CMM SP $r$ depends not only on the service price $x_{r}$ and the cost $c_{r}$, but also on the service prices $x_{-r}$ offered by the other cloud SPs [46].

Each CMM SP decides its action independently and simultaneously. And the CMM SP with the lowest price will occupy the entire service market. Hence, every CMM SP tries to reduce its service price until hitting the bottom with zero profit.

As discussed in Section 3.1, the set of the game players is $\mathbf{R}=\{1, \ldots, r, \ldots, \mathcal{R}\}$, the strategy set is $x_{r}$, and the payoff function of the CMM SP is $U_{r}$. The NE of this problem gives the set of prices such that neither CMM SP can increase its net profit $U_{r}$ by unilaterally changing the price. Without loss of generality, let the cost set in an ascending order $c_{1}<c_{2}<\ldots<c_{\mathcal{R}}$.

Proposition 3.2.1. The NE of the proposed homogeneous Bertrand game with multiple CMM SPs is shown as below,

$$
x^{*}=\left\{x_{1}^{*}, c_{2}, c_{3}, \ldots, c_{\mathcal{R}}\right\},
$$


where $x_{1}^{*}$ denotes the price strategy of the first CMM SP at the Nash-equilibrium, which can be formulated as,

$$
x_{1}^{*}=\arg \max _{c_{1} \leq x_{1}<c_{2}} U_{1}^{M}\left(x_{1}\right)
$$

where $U_{1}^{M}$ is the utility function of the provider 1 when it supplies the whole market, shown as (3.28).

$U_{1}^{M}=\left(x_{1}-c_{1}\right)\left(B_{r m}\left[W \log _{2}\left(1+\frac{p_{m} h_{m}^{2}}{I_{m}}\right)\right]^{+}+\sum_{k=1}^{K} B_{r k}\left[W \log _{2}\left(\frac{h_{k}^{2} W\left(1-\mu_{k} x_{1} B_{r k}\right)}{\ln 2 \lambda_{k} y g_{k m}^{2} \sigma_{k}^{2}}\right)\right]^{+}\right)$

Proof. By observing the (3.19), we know that $U_{1}^{M}$ is the utility function of $x_{1}$. Assume in a Bertrand condition, there are only two CMM SPs, the provider 1 and the provider 2 in the competition. The costs are defined as $c_{1}$ and $c_{2}$, and we let $c_{1}<c_{2}$. According to the assumption about the Bertrand competition model, both providers have the incentives to reduce their service prices down to their own margin cost to undercut the other and capture the whole market to almost double its profit. That is to say, if one provider sets its price equals to the marginal cost, and the other provider tries to raise its service price over the cost, then it will earn nothing. Since all the endusers would purchase the service from the provider that is still setting the competitive price. If one provider has a minor average cost, it will charge the highest price that is lower than the average cost of the other one and takes all the business. So provider 1 has the incentive to make its price between the set of $\left[c_{1}, c_{2}\right)$ to maximize its own profit.

Though it is irrational to set the price below the marginal cost, the two CMM 
SPs would make the prices lower than their own monopoly price. If the provider 2 presents a high enough price, the provider 1 can definitely ignore the affection of provider 2 and set its price by the optimal monopoly price.

Due to the piecewise property about $x_{1}$, to maximize the utility function $U_{1}^{M}$, for all $k \in\{1,2, \ldots, K\}$, we introduce the indicator function as below,

$$
\begin{aligned}
& V_{m}= \begin{cases}1, & x_{1}<\frac{W-\ln 2 \lambda_{k} y\left[\frac{1}{K}\left(p_{m} h_{m}^{2}-\sigma_{m}^{2}\right)+g_{k m}^{2} \frac{\sigma_{k}^{2}}{h_{k}^{2}}\right]}{W \mu_{k} B_{r k}}, \forall k \\
0, & \text { otherwise. }\end{cases} \\
& V_{k}= \begin{cases}1, & x_{1}<\frac{W h_{k}^{2}-\ln 2 \lambda_{k} y g_{k m}^{2} \sigma_{k}^{2}}{W h_{k}^{2} \mu_{k} B_{r k}}, \forall k \\
0, & \text { otherwise. }\end{cases}
\end{aligned}
$$

Thus by substituting the (3.29) and (3.30) into (3.28), we can know that the utility function is a piecewise function about $x_{1}$ due to those indicator functions $V_{m}$ and $V_{k}$, which cannot obtain the derivative directly to solve $x_{1}$. However, if determined $V_{m}$ and $V_{k}$, we still can get a continue differentiable function.

Therefore we let,

$$
\begin{aligned}
& F_{m}=\frac{W-\ln 2 \lambda_{k} y\left[\frac{1}{K}\left(p_{m} h_{m}^{2}-\sigma_{m}^{2}\right)+g_{k m}^{2} \frac{\sigma_{k}^{2}}{h_{k}^{2}}\right]}{W \mu_{k} B_{r k}}, \\
& F_{k}=\frac{W h_{k}^{2}-\ln 2 \lambda_{k} y g_{k m}^{2} \sigma_{k}^{2}}{W h_{k}^{2} \mu_{k} B_{r k}} .
\end{aligned}
$$

Then sort all $F_{1}, F_{2}, \ldots, F_{m}$, and $F_{k}$ in an ascending order $F_{1} \leq F_{2} \leq \ldots F_{m} \leq F_{k}$, 
without loss of generality. Hence we can get $K$ intervals, $\left[0, F_{1}\right),\left(F_{2}, F_{3}\right), \ldots,\left(F_{m}, F_{k}\right)$. By piecewise differentiating of the utility function in the first interval, we assume that $0<x_{1}<F_{1}$, the second derivative of $U_{1}^{M}\left(x_{1}\right)$ is shown as (3.33).

$$
\begin{aligned}
\frac{\partial^{2} U_{1}^{M}\left(x_{1}\right)}{\partial x_{1}^{2}}= & 2\left\{\sum_{k=1}^{K} \frac{W^{2} \mu_{k} B_{r k} B_{r m} p_{m} h_{m}^{2}}{(\ln 2)^{2} \lambda_{k} y I_{m}\left(p_{m} h_{m}^{2}+I_{m}\right)}-\sum_{k=1}^{K} \frac{B_{r k}^{2} W \mu_{k}}{\ln 2\left(1-\mu_{k} x_{1} B_{r k}\right)}\right\} \\
& +\left(x_{1}-c_{1}\right)\left\{\sum_{k=1}^{K} \frac{W^{3} \mu_{k}^{2} B_{r k}^{2} B_{r m} p_{m} h_{m}^{2}\left(p_{m} h_{m}^{2}+2 I_{m}\right)}{(\ln 2)^{3} \lambda_{k} y I_{m}^{2}\left(p_{m} h_{m}^{2}+I_{m}\right)^{2}}-\sum_{k=1}^{K} \frac{W B_{r k}^{3} \mu_{k}^{2}}{\ln 2\left(1-\mu_{k} x_{1} B_{r k}\right)^{2}}\right\} .
\end{aligned}
$$

We have known the definition of $I_{m}$ from (3.13), for substituting it into (3.33), we can obtain that the second derivative $\frac{\partial^{2} U_{1}^{M}\left(x_{1}\right)}{\partial x_{1}{ }^{2}}$ is less than 0 , which means that $\frac{\partial U_{1}^{M}\left(x_{1}\right)}{\partial x_{1}}$ is a monotonic decreasing function of $x_{1}$. Obviously, we obtain the function $(3.34)$.

$$
\begin{aligned}
\frac{\partial U_{1}^{M}\left(x_{1}\right)}{\partial x_{1}}= & B_{r m} W \log _{2}\left(1+\frac{p_{m} h_{m}^{2}}{I_{m}}\right)+\sum_{k=1}^{K} B_{r k} W \log _{2} \frac{W h_{k}^{2}\left(1-\mu_{k} x_{1} B_{r k}\right)}{\ln 2 \lambda_{k} y g_{k m}^{2} \sigma_{k}^{2}} \\
& +\left(x_{1}-c_{1}\right)\left\{\sum_{k=1}^{K} \frac{W^{2} \mu_{k} B_{r k} B_{r m} p_{m} h_{m}^{2}}{(\ln 2)^{2} \lambda_{k} y I_{m}\left(p_{m} h_{m}^{2}+I_{m}\right)}-\sum_{k=1}^{K} \frac{B_{r k}^{2} W \mu_{k}}{\ln 2\left(1-\mu_{k} x_{1} B_{r k}\right)}\right\} .
\end{aligned}
$$

From (3.34), we have,

$$
\lim _{x_{1} \rightarrow 0} \frac{\partial U_{1}^{M}\left(x_{1}\right)}{\partial x_{1}}>0 .
$$

When $x \rightarrow F_{1}$, we can get two cases as follows:

$$
\begin{aligned}
& \text { (1) } \lim _{x \rightarrow F_{1}} \frac{\partial U_{1}^{M}\left(x_{1}\right)}{\partial x_{1}} \geq 0 ; \\
& \text { (2) } \lim _{x \rightarrow F_{1}} \frac{\partial U_{1}^{M}\left(x_{1}\right)}{\partial x_{1}}<0 .
\end{aligned}
$$


For the first case, the utility $U_{1}^{M}$ is strictly monotonic increasing about $x_{1}$ at the initial interval $\left[0, F_{1}\right)$. Therefore, for the second case, we know that the utility function $U_{1}^{M}$ firstly climbs up with $x_{1}$. After reaching the optimal point, it drops down with $x_{1}$. Hence, the utility function $U_{1}^{M}$ is a concave function at the first interval, and it is easily to prove the utility function $U_{1}^{M}$ is the concave function at the other intervals. That is to say, for $x_{1}<F_{k}$, the utility function is a concave function without the most $K$ non-differentiable points at $F_{1}, F_{2}, \ldots, F_{m}$, and $F_{k}$. We can solve $x_{1}$ at each interval by many methods (e.g., a binary search algorithm and a gradient based algorithm).

\subsubsection{Service Allocation Iteration Algorithm}

It is important to investigate the uniqueness and the existence of the Stackelberg equilibrium. In the duopoly case, the convexity of the follower's reaction function is essential for uniqueness of the Stackelberg equilibrium [47]. Hence, we will prove that for our model of Stackelberg game has an unique equilibrium.

Theorem 3.1. The unique Nash equilibrium exists in the proposed Stackelberg game.

Proof. In our Stackelberg game model, each stage has its flawless equilibrium in a Nash equilibrium respectively: the service price strategies $x^{*}$ in (3.26) offered by the CMM SPs, the service allocation strategy $s_{m}^{*}$, the interference price, and the service allocation strategy $s_{k}^{*}$ in (3.10). Because we have proven that each stage exists a perfect equilibrium in a Nash equilibrium, the Nash equilibrium of the proposed Stackelberg game model exists. We also know that the subgame perfect equilibrium in each stage is unique. Therefore, the total Stackelberg Nash equilibrium is unique.

To get the Nash equilibrium of the three-stage Stackelberg game, we use a backward induction to solve the problem and present the service iteration algorithm.

In the above method, we defined the other CMM SPs' strategies as $x_{-r}=$ $\left(x_{1}, x_{2}, \ldots, x_{r-1}, x_{r+1}, \ldots, x_{R}\right)$. When $x_{-r}$ is given at iteration $t-1$, we present the 


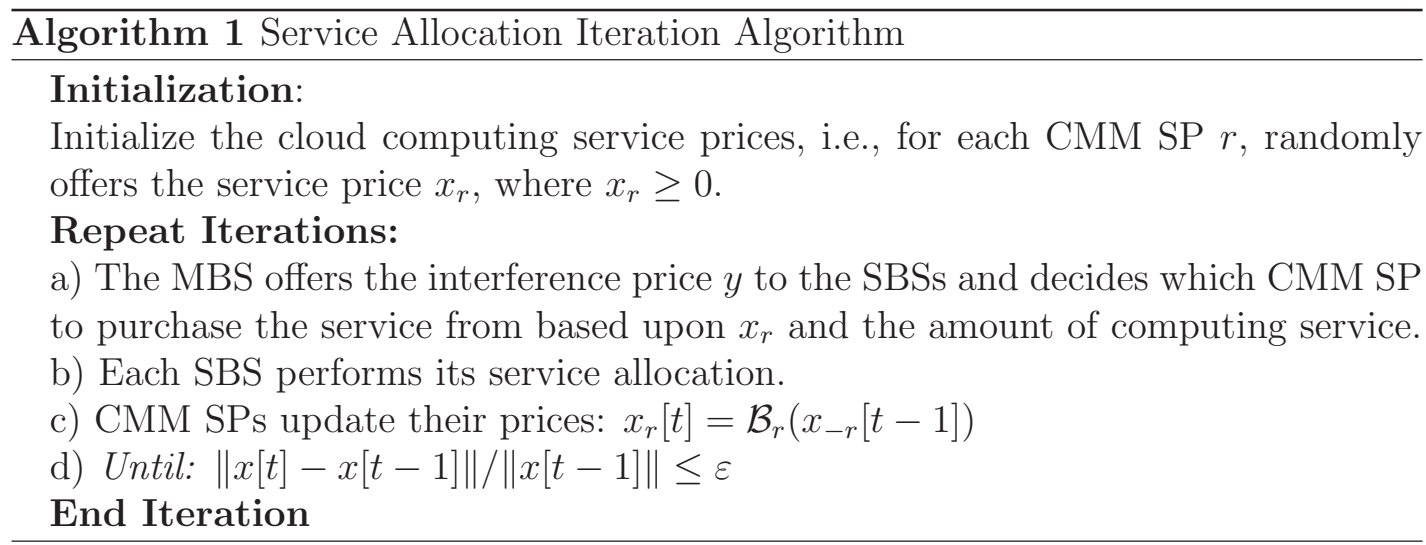

best response function $\mathcal{B}_{r}\left(x_{-r}[t-1]\right)$ of CMM SP $x_{r}$ at the iteration $t$ to maximize its total revenue. The condition $\|x[t]-x[t-1]\| /\|x[t-1]\| \leq \varepsilon$ is the stop criteria. In the proposed algorithm, the MBS decides the interference price $y$ offering to SBSs and the amount of service purchased from the CMM SPs based on the service price $x_{r}$. The SBS then allocates the power. The algorithm will stop until the service price $x_{r}$ converges.

In practice, the proposed iterative algorithm to obatin the threee-stage Stackelberg game equilibrium can be implemented as follows,

1. The CMM SPs randomly offer the CMM service price to the MBS and SBSs.

2. The MBS receives the channel state information from the SBSs and the MUs.

3. The MBS decides which provider to purchase the service from and the interference price offering to the SBSs.

4. The SBSs perform their power allocation.

5. The CMM SPs update their prices and repeat steps 2, 3 and 4 until the prices converge.

To ensure the convergence to the NE for the Algorithm 1, some sufficient conditions have been proposed in the existing literature. The convergence condition was first 
provided by [48] for the two-user case and extended for the N-users in [49]. Moreover, the conditions of the convergence were further proved in $[50,51]$. However, as the pricing factor $x_{r}$ is recalculated in every iteration, the algorithm is actually a timevarying over iterations. Thus the fixed-point theorem proposed in $[50,51]$ may not be applied here. The convergence proof under a time-varying mapping function is a challenging problem and will be left for the future work. Nevertheless, convergence has always been observed in our simulations.

\subsection{Simulation Results and Discussions}

In this section, we use computer simulations to evaluate the performance of the proposed scheme by MATLAB software. All the simluations are executed on a laptop featured with Windows 7, Intel Core i5 2.6GHz CPU, 8GB memory and MATLAB R2012a. The main system parameters in HWNs are adopted from 3GPP [44], as listed in Table 3.2. In the simulations, we assume that there are 2 CMM SPs. The MUs and small cells are located in the macrocell randomly, and small cells deploy sparsely with each other at $50 \mathrm{~m}$ to $150 \mathrm{~m}$ far from the MBS. Following [44], we set the path loss between SSU and MBS as $15.3+37.6 \log _{10}\left(D_{m s}+L_{o w}\right)$, and the path loss between SSU and SBS as $46.86+20 \log _{10}\left(D_{s s}+L_{i w}\right) . D_{m s}$ is the distance between the MBS and the SSU. $D_{s s}$ is the distance between the SBS and the SSU. $L_{o w}$ means the penetration loss of exterior wall, and $L_{i w}$ means the penetration loss of the interior wall. They are set as $20 d B$ and $10 d B$, respectively. We can also find the parameters of the small scale and shadow fading in [44]. The others are shown as below. The transmission bandwidth $W$ is $5 \mathrm{MHz}$ in the MBS and the transmission power is fixed as $46 \mathrm{dBm}$ from [44]. The general parameters are set as, $\mu_{k}=0.05, \lambda_{k}=1, \alpha=0.03$, $\beta=10, B_{r k}$ and $B_{r m}=1$.

Firstly, we evaluate the performance of the CMM service purchased by BSs with 
various lowest prices. Fig. 3.4 shows that, with the increase of the lowest service price $x_{r}, S B S_{1}$ and $S B S_{2}$ have to decrease their transmission rate by performing the energy-efficiency power allocation. Due to the fixed transmission power of the MBS, it performs an increasing trend with $x_{r}^{*}$. The reason is that, by increasing $x_{r}^{*}$, the transmission power of SBS decreases as well, which in turn leads to the decrease of interference between the MBS and the SBS. Hence, the transmission rate of the MBS has the same variation trend with the service price $x_{r}^{*}$. When the service price is too high to afford for the SSUs, the SBSs will lower their transmission rate step by step until stop transmitting anything. Because the SBSs stop their transmission, the SSUs choose another method to receive the CMM service due to the high service prices. The MBS will reach its highest level of the transmission rate shown in Fig. 3.4. The shape of the curve can change with the parameters. However, the insight remains the same in figure.

Then we compare the CMM service purchased by one SBS with three different values of $x_{r}^{*}$. Fig. 3.5 shows that the SBS tries to reduce its interference cost $y$ by decreasing its transmission rate $s_{k}$ when given the value of service price offered by the CMM SP. Fig. 3.5 also shows that, in the condition of the same interference price, the lower service price offered by the CMM SPs, the higher transmission rate we can obtain.

We also study the service allocation in the MBS with various service prices offered by the CMM SPs in Fig. 3.6. The interference between MBS and SBSs will be reduced by the increasing trend of the service price $x_{r}$. That is because the transmission power $p_{m}$ is fixed in the MBS, the transmission rate of MBS will increase with the service price until it reaches the highest level, then the interference turns to zero. We can find how the value of $\alpha$ effects the shape of the transmission rate $s_{k}$ in MBS, the smaller the value of $\alpha$, the higher level of transmission rate we can obtain. As $\alpha$ increases, 
Table 3.2: Simulation Parameters and Assumptions for Performance Evaluations

\begin{tabular}{|c|c|}
\hline Parameters & Values / Assumptions \\
\hline \hline $\begin{array}{c}\text { Deployment of stand-alone } \\
\text { small cells }\end{array}$ & $\begin{array}{c}\text { Randomly deployed at } 50 \mathrm{~m} \text { to } \\
150 \mathrm{~m} \text { far from the MBS }\end{array}$ \\
\hline Path loss: MBS $\leftrightarrow \mathrm{SSU}$ & $15.3+37.6 \log _{10} D_{m s}+L_{o w}{ }^{2}$ \\
\hline Path loss: SSU $\leftrightarrow$ SBS & $46.86+20 \log _{10} D_{s s}+L_{i w}{ }^{2}$ \\
\hline Penetration loss of exterior wall & $20 \mathrm{~dB}$ \\
\hline$L_{o w}$ & $10 \mathrm{~dB}$ \\
\hline Penetration loss of interior wall & $5 \mathrm{MHz}$ \\
\hline The MBS transmission bandwidth & $46 \mathrm{dm}$ \\
\hline MBS TX power & 50 \\
\hline Number of sub-channel in each BS & $1000 \mathrm{~m}$ \\
\hline Diameter of Macro-cell coverage & $20 \mathrm{~m}$ \\
\hline Diameter of Small cell coverage & $>5 \mathrm{~m}$ \\
\hline Minimum distances among & \\
stand-alone small cells & \\
\hline \hline
\end{tabular}

${ }^{1} D_{m s}$ is the distance between the MBS and the SSU

${ }^{2} D_{s s}$ is the distance between the SBS and the SSU 


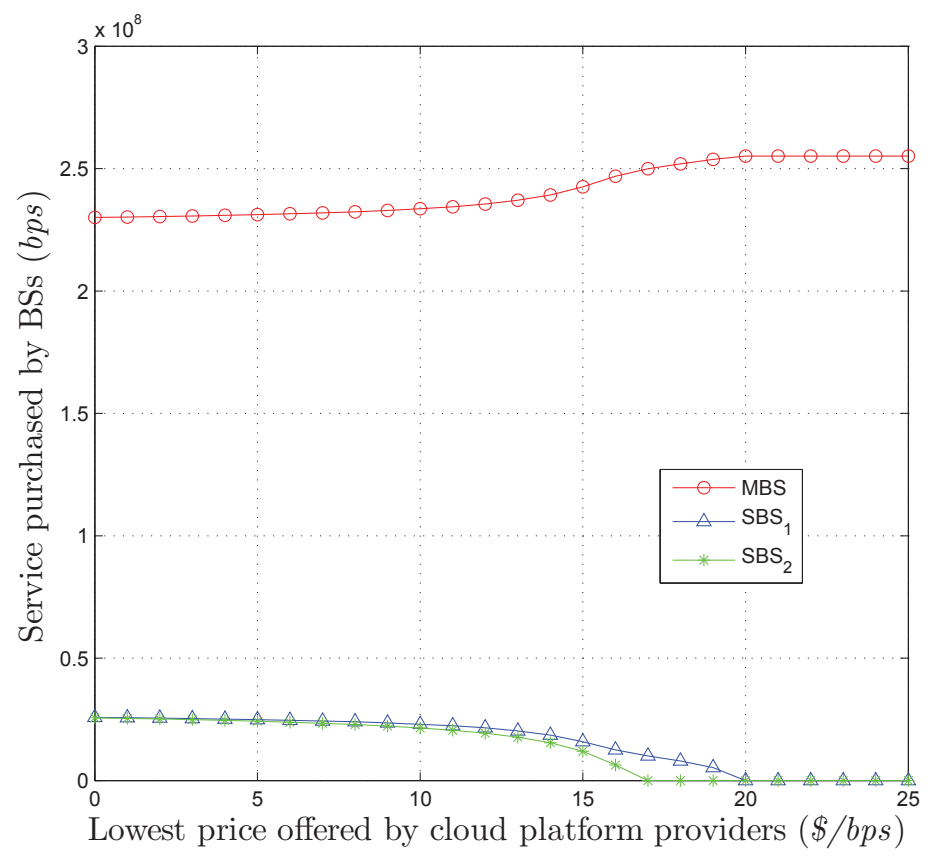

Figure 3.4: Service allocation with various lowest prices offered by the CMM service providers.

there is tradeoff among the transmission rate, service cost and interference revenue in the MBS.

Fig. 3.7 shows that the utility function of SBS is a concave function, which is proven in Subsection 3.2.1. In this figure, we know that the utility of SBS, firstly, increases as the power. After it reaches the optimal level, the utility of SBS begins to decrease, because the gain of the transmission rate cannot offset the increase trend of the service cost and interference price. This figure also tells us that the higher the interference price is, the lower utility of SBS will be.

In addition, we find that the transmission rate of the MBS correspondingly increases with the interference price in Fig. 3.8. That is because the higher interference price forces the SBSs to reduce their transmission power. We also observe that the higher the service price $x_{r}^{*}$ is, the lower the interference from the SBSs will be. Hence, 


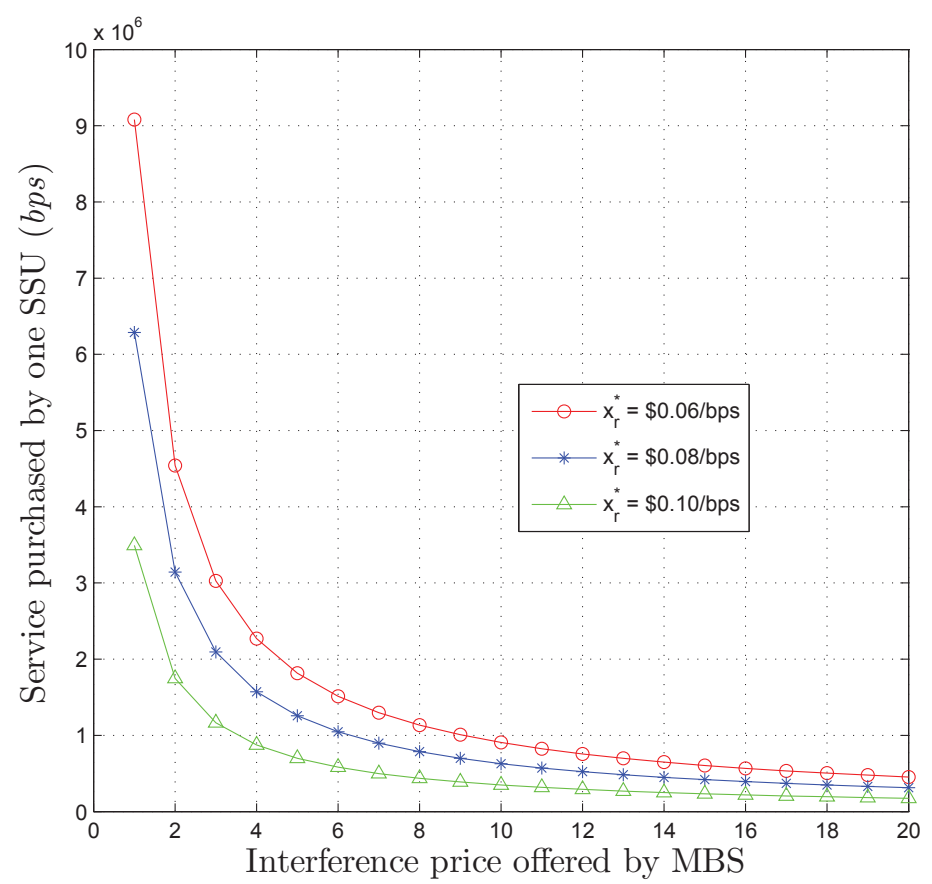

Figure 3.5: Service allocation in one SBS with various lowest service prices offered by the MBS.

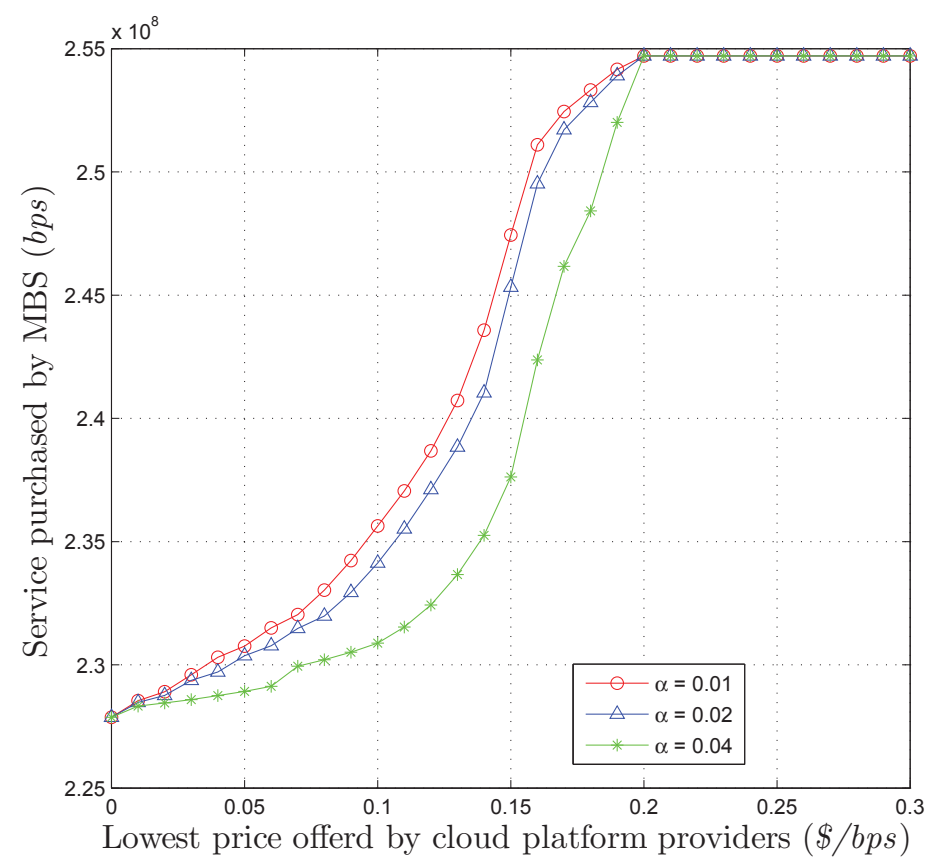

Figure 3.6: Service allocation in the MBS with various values of $\alpha$ parameter. 


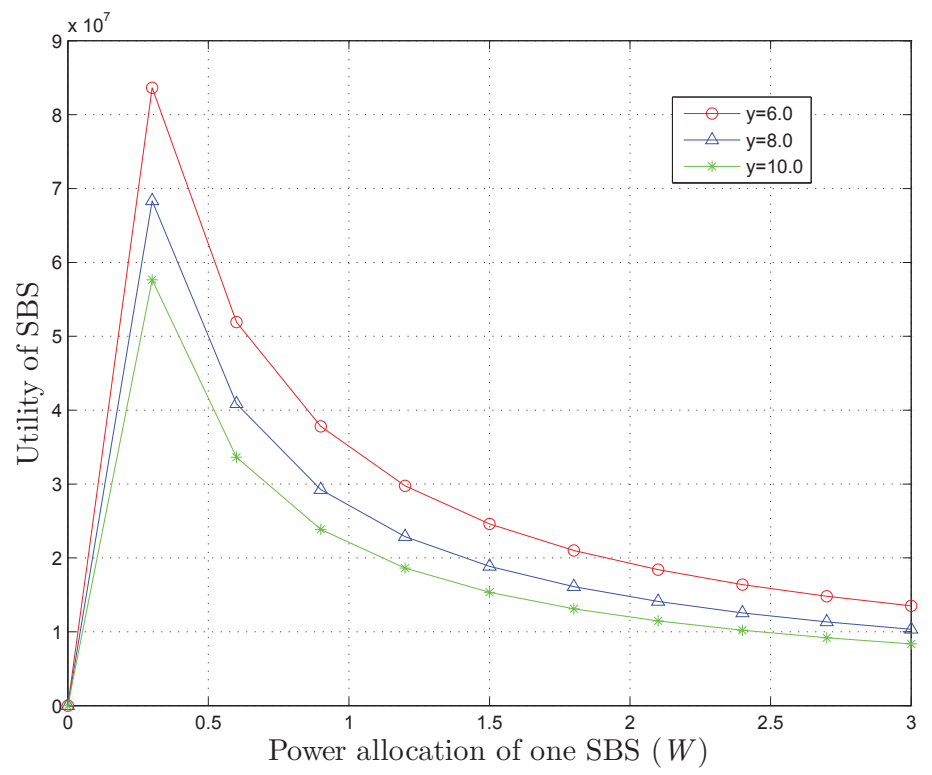

Figure 3.7: Utility function of one SBS.

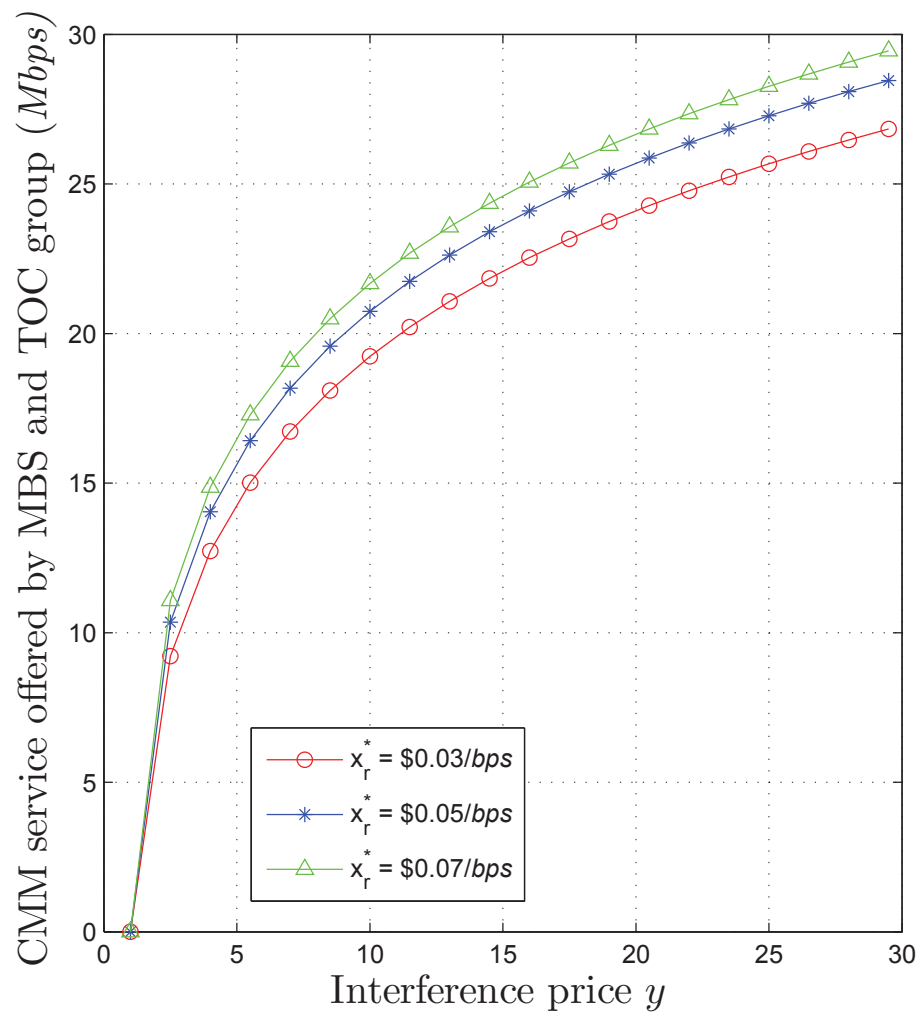

Figure 3.8: The performance of CMM service in the MBS. 


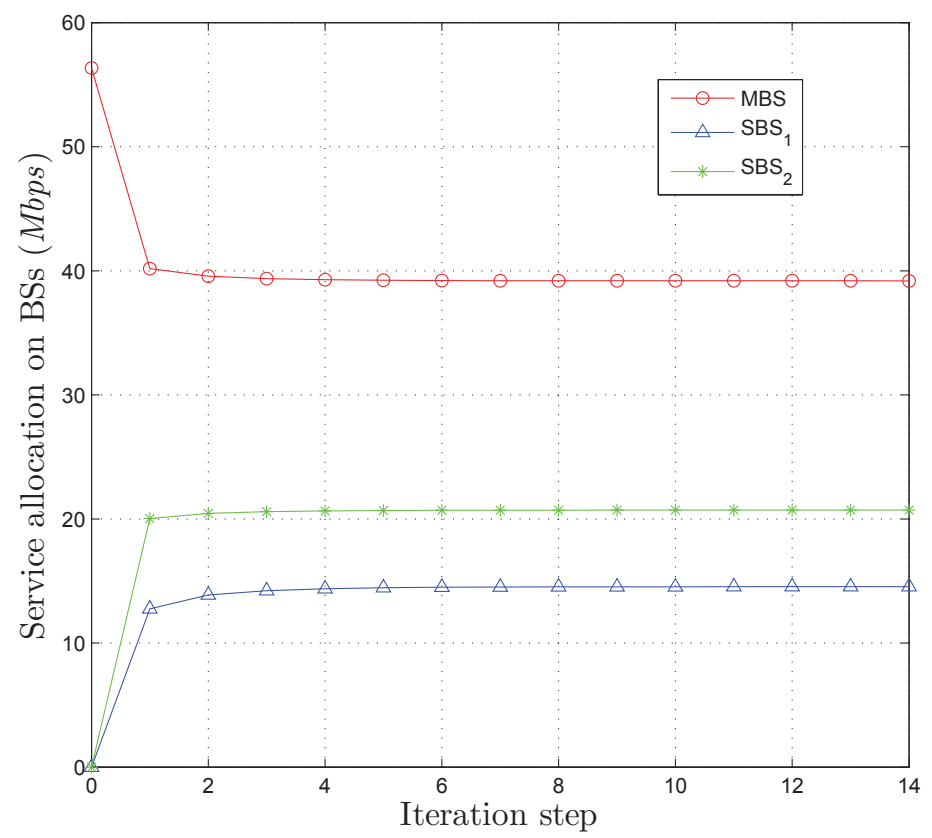

Figure 3.9: CMM service iteration step.

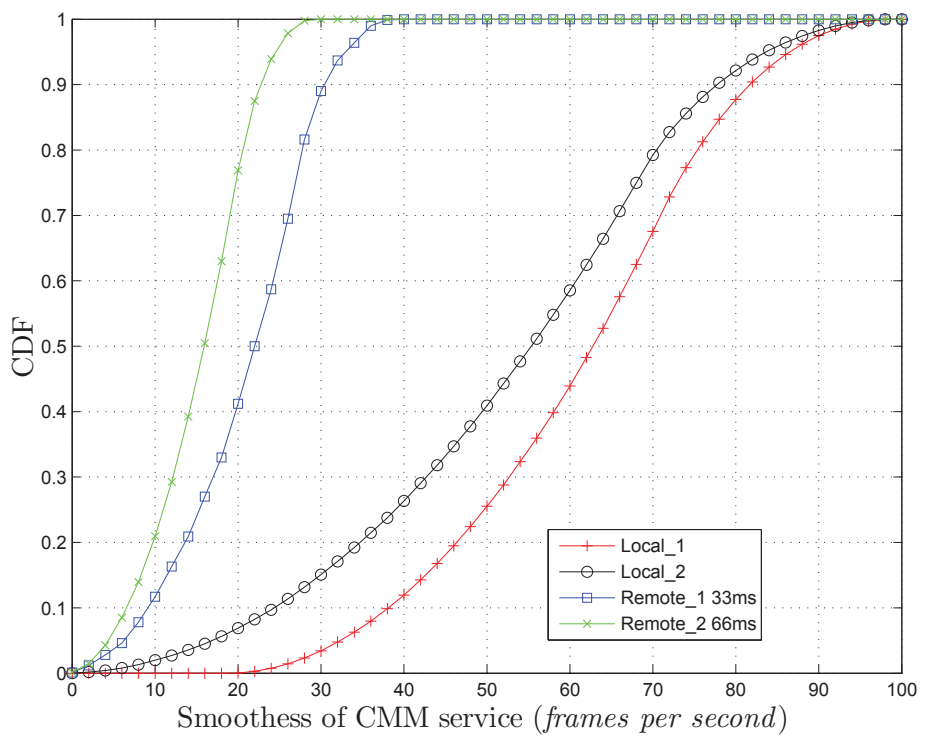

Figure 3.10: Network latency hurts interactive performance even with good bandwidth. 


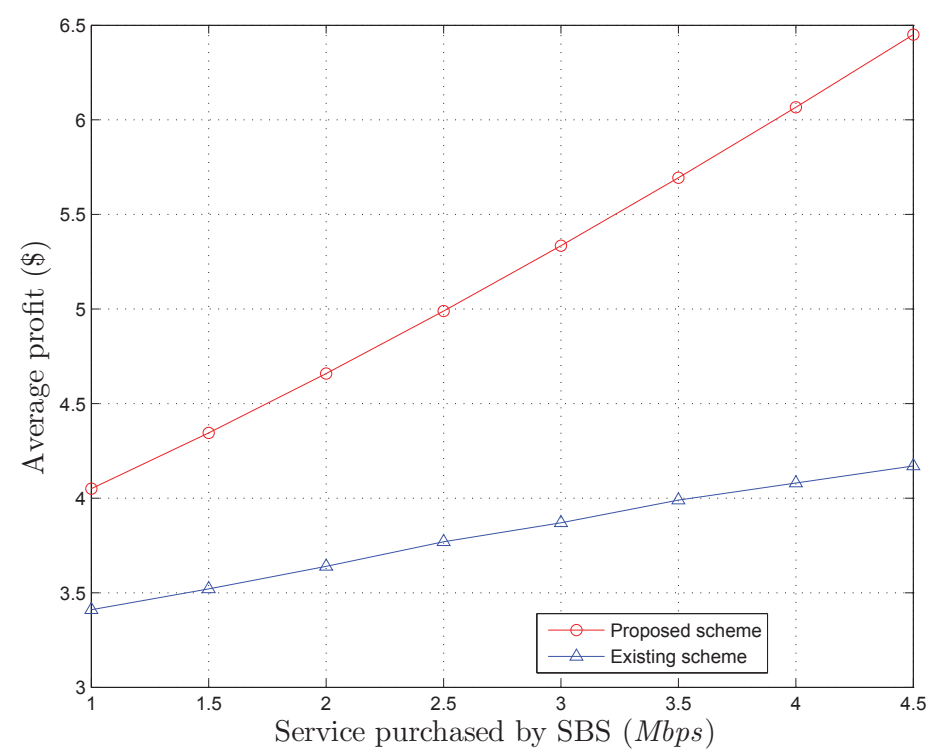

Figure 3.11: The average profit versus service allocation on one SBS.

the transmission rate will reach a higher level in the MBS with a more expensive service price offered by the CMM SPs.

Fig. 3.9 shows the convergence of the proposed Stackelberg equilibrium iteration algorithm. We evaluate the performance of the CMM service over the iteration steps. From the figure, we can observe the service of MBS and SBSs can converge after a few iteration steps because of the convergence of the service price $x_{r}^{*}$. Hence we will obtain the NE by the algorithm.

Then, we study the frame rate (frames per second) and latency of CMM service in the mobile cloud computing environment under two different configurations: the local servers and the remote cloud computing servers over a $100 \mathrm{Mb} / \mathrm{s}$ network with the output viewed through the virtual network computing (VNC) protocol, which have the different latency due to the different distances among the remote servers. Fig. 3.10 demonstrates that a high frame rate provides the illusion of smoothness to an enduser. Even a modest latency of $33 \mathrm{~ms}$ can cause the frame rate to drop dramatically from that experienced with a local server. Although the VNC protocol strives to keep 
the frame rate at an acceptable level, it offers sluggish interaction. Hence, the user experience is considerably poorer than that for the local media service interaction.

Finally, the average profit for our proposed scheme compared with an existing scheme [52] is evaluated in Fig. 3.11. In the existing scheme, multiple services prices are offered to the SUs. This service pricing scheme adopts the non-incentive compatible differentiated type, which can show the theoretical upper bound of the overall profit of this method. The transmission rate is controlled between 1 and 4.5 Mbps. We recalculate the profits of the CMM SPs 1000 times at each 0.5 Mbps interval, then obtain the average profits. In the figure, we compare the average profit in our scheme and the existing scheme based on the CMM service purchased by the SBSs. We can find that the profits in the two schemes both have the growing trends with the increasing CMM service purchased by the SBS. Simulation results show that the proposed scheme can gain more profit than the existing one in the same service allocation condition. That is because the existing scheme does not consider the dynamic resource allocation method.

\subsection{Chapter Summary}

In this chapter, we have studied the issues that arise when jointly considering the operations of cloud and wireless networks in a mobile cloud computing environment with a telecom operator cloud. We introduced a system model, which jointly considers the CMM SPs and HWNs with small cells. Multiple CMM SPs offer CMM service prices to the heterogeneous networks. Then the MBS and SBSs adjust the amount of service they procured by performing resource allocation. We formulated the problems of determining a CMM service price, wireless power allocation, and interference management as a three-level Stackelberg game. We also presented an interference price to measure and mitigate the cross-tier interference between the macrocell and 
small cells. The MBS is allowed to protect its own users by charging the SBSs. At the CMM SP's level, we proposed a homogeneous Bertrand game with asymmetric costs to model the CMM service decisions and used a backward induction method to solve the whole model. Finally, we presented an iteration algorithm to obtain the equilibrium of the Stackelberg Game. Simulation results have been presented to show that the dynamics of cloud operations have a significant impact on the heterogeneous wireless network, and joint optimization is necessary for the operations of cloud and wireless networks. This is due to unique dynamics tied with cloud, CMM services and wireless networks. By jointly optimizing the operations of clouds and wireless networks, the proposed scheme can significantly improve the performance of the mobile cloud computing systems.

In the next chapter, we will extend our mobile cloud computing model and discuss the effectiveness of this new game-theoretic approach. 


\section{Chapter 4}

\section{Cloud and Wireless Network Operations through Network Virtualization}

\subsection{System and Description}

In this chapter, we focus on jointly considering the cloud and the HWNs techniques, therefore the end-users can experience less response time and higher QoS of CMM services in a mobile cloud computing environment [10, 18, 24, 26, 53-58]. By exploiting the unique structure of mobile cloud computing networks, we jointly study the operations of clouds and the HWNs with the goal of improving CMM service performance. The contributions of this chapter are summarized as follows:

- We extend the system model with multiple groups of MBS and TOC through network virtualization technology.

- We observe the problems of determining CMM services price decision, allocation of wireless resources, and interference management. This problem is then formulated as a three-level Stackelberg game, which includes the following:

1. First level: a homogenous Bertrand game that determines the CMM service price decision; 
2. Second level: the noncooperative game between the MBS and TOC groups;

3. Third level: an evolutionary game between the SBSs.

- To analyze the proposed game, we use the backward induction method to capture the sequential dependencies of decisions on the different levels of the game. We perform a theoretical analysis of the three stages of the game to obtain an equilibrium at each level. We also present the algorithms for each level to achieve the equilibrium states.

- Extensive simulations are presented to demonstrate the performance of the proposed approach. Based on the simulation results, we verify the convergence of the Stackelberg equilibrium iteration algorithm.

The rest of this chapter is organized as follows. Section 4.2 analyzes the proposed game. Section 4.3 presents the implementation of the proposed protocols and the algorithms to reach the Stackelberg game equilibrium states. The simulation results are presented and discussed in section 4.4.

Fig.4.1 shows the mobile cloud computing model with the CMM SPs, telecom operators with cloud, and HWNs with macrocell and small cell base stations. In this section, we firstly, describe the cloud platform model, and then we discuss HWNs. In addition, we describe how the operations of clouds and wireless networks in a mobile cloud computing system are formulated using a multi-level Stackelberg game.

\subsubsection{Cloud Platform Model with Network Virtualization Technology}

The concept of the telecom operator cloud has been introduced in the previous chapter. Telecom networks have various functions and network elements that could be implemented in a cloud, called a telecom operator cloud. The operators can fully 


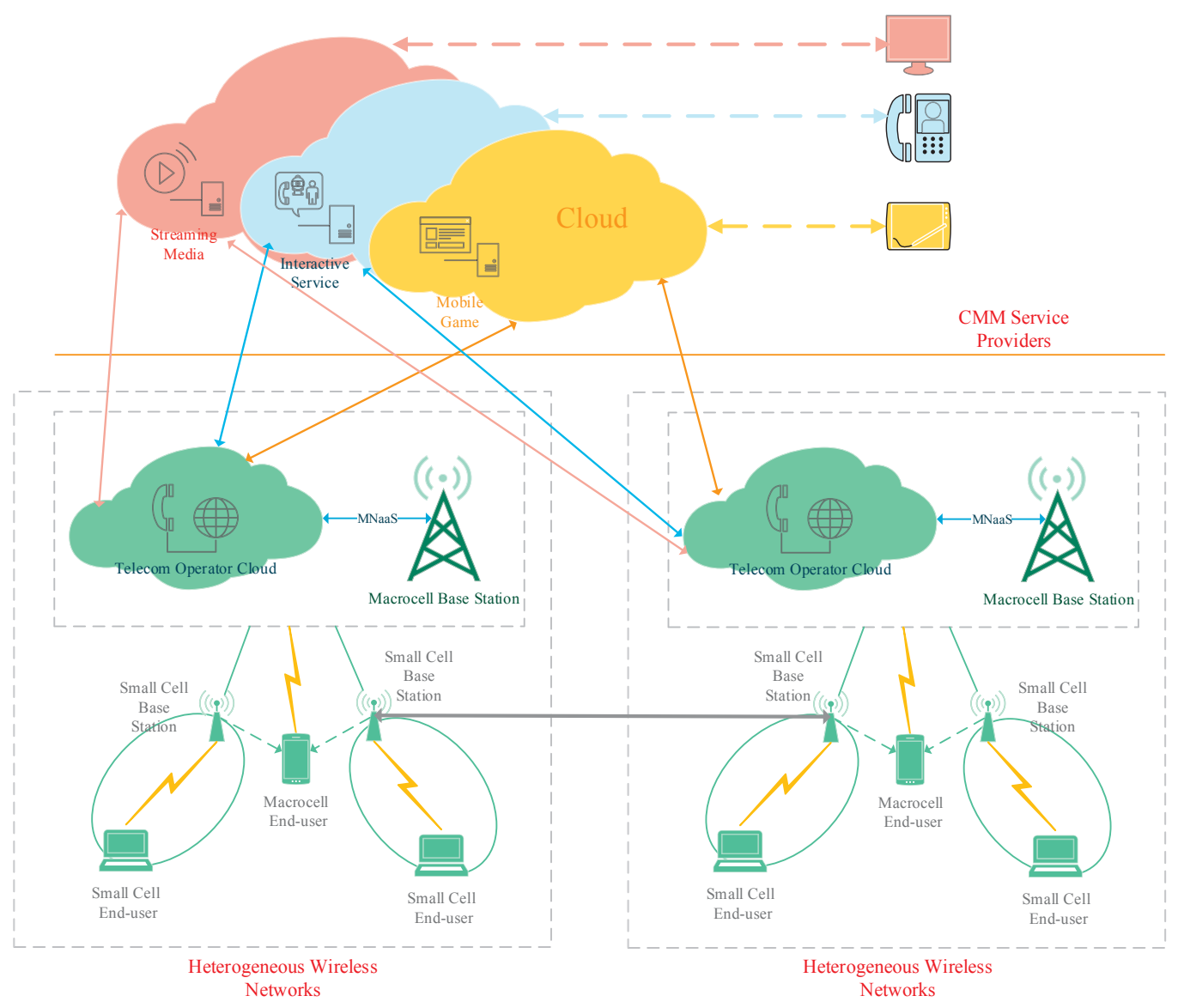

Figure 4.1: Mobile cloud computing model with HWNs. 
Third Party CMM Service Providers

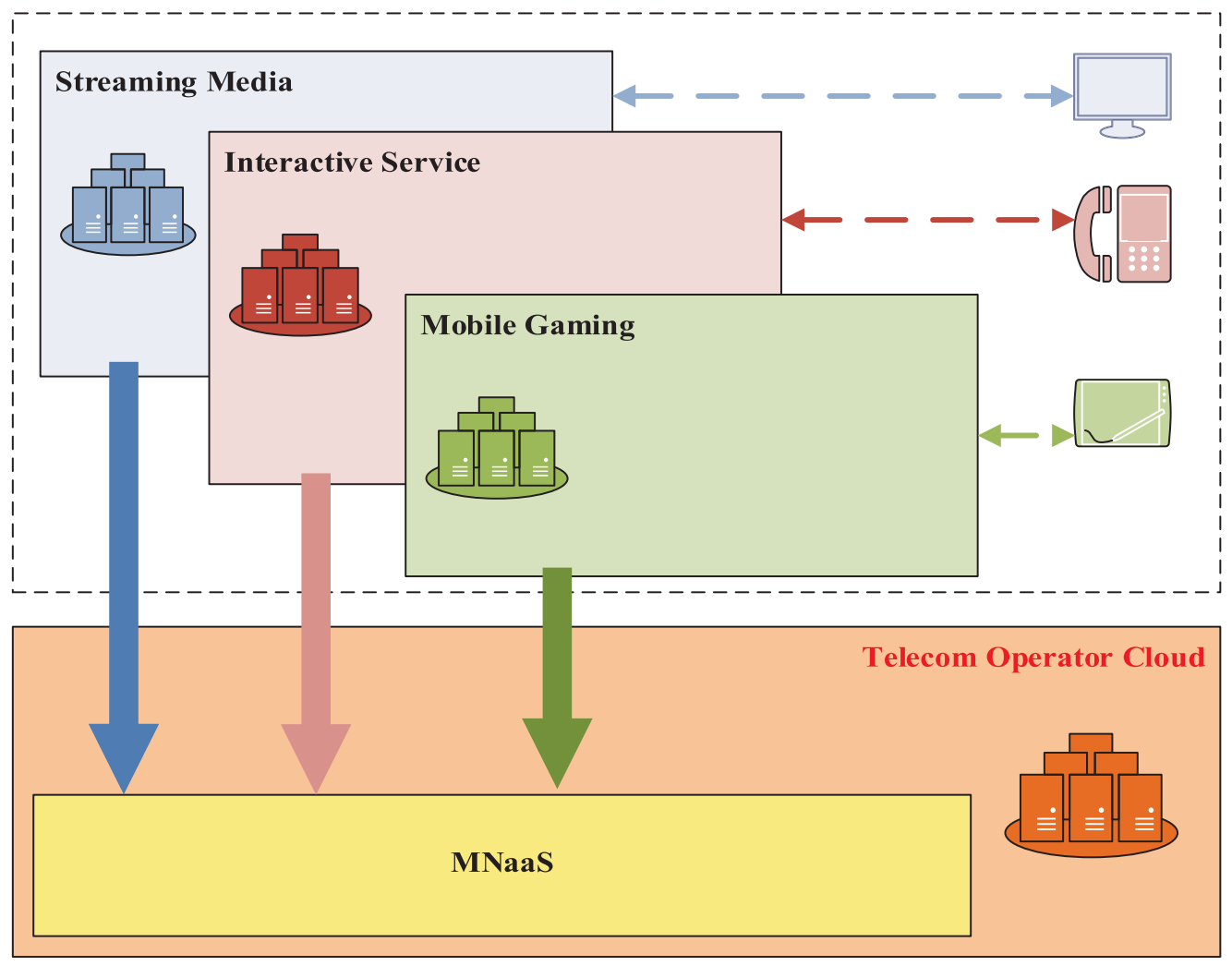

Figure 4.2: CMM service model.

utilize all kinds of cloud computing services like IaaS, SaaS, and PaaS. The operators can also consider the MNaaS concept. This means that operators can open up their networks' assets to the CMM SPs and divide the physical substrate networks into some virtual wireless network resources by using virtualization technology. Briefly, the CMM SPs can lease these virtual resources and then operate and program them to satisfy the requirements of the end users. Therefore, in this scenario, TOCs can share information and play roles as cloud "brokers" between the end users and the third party CMM SPs regardless of where they are. 
The focus of the communications business has undergone a fundamental shift, with basic network features such as coverage and availability no longer enough to differentiate operators in an increasingly crowded and dynamic marketplace. At the same time, the boom in data traffic volumes is putting more pressure than ever on operators to invest in network capacity while driving down the cost per delivered bit. Especially for the needs of entertainment, customers are more dynamic and unpredictable from a telecom operator's perspective. An end-user may require a high bandwidth for a limited time span, or may want to add or withdraw new locations within their wireless network. This creates a range of different demand patterns and service level agreements on the same network. Hence, in this chapter, we adopt the MNaaS business model shown in Fig. 4.3 to virtualize our network to enable the telecom operators to deliver a wide variety of high quality media services for endusers, and to explore new business models and drive down costs.

\subsubsection{Heterogeneous Wireless Networks}

Our proposed HWNs architecture is described in the previous chapter. This architecture comprises three main components, including MBS, SBS, and end-users. In this chapter, small cells are defined as wireless infrastructure equipment, which operate in licensed bands, such as femtocells, picocells, microcells, and metrocells. There are multiple SBSs and MBSs in the entire architecture. Each SBS is connected to one MBS via a broadband connection such as DSL or a cable modem. MBS is set up by the telecom operator. The MBS and the small cells have a cognitive capability, which means that they can sense the channel state information. MBS also comprises multiple MUs. To simplify the analysis, without loss of generality, we assume that

in each small cell there is only one SSU. All the small cells are deployed sparsely to avoid mutual interference with each other. The whole system is operated in a time 
MNaaS Model

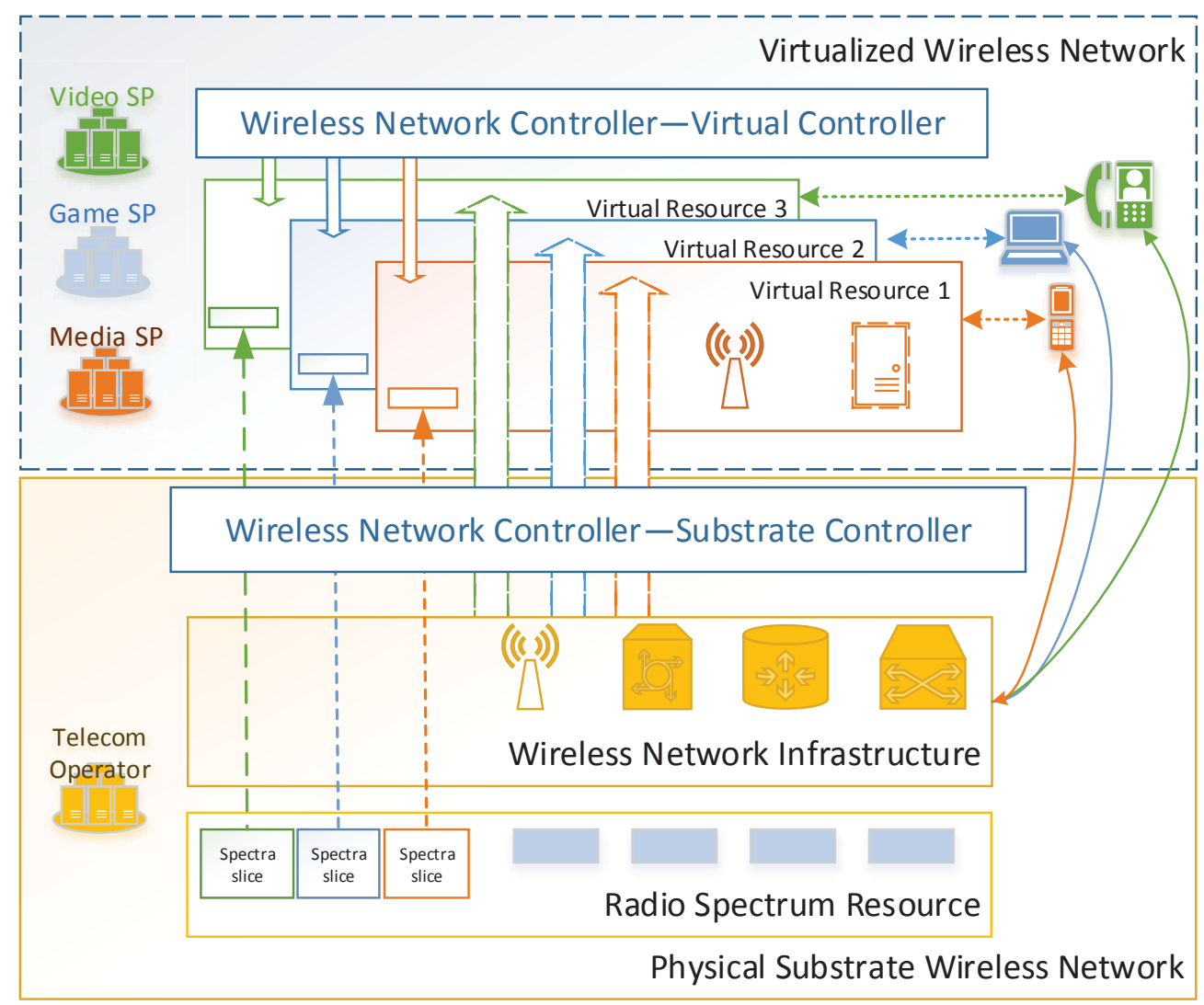

Figure 4.3: The MNaaS model. 


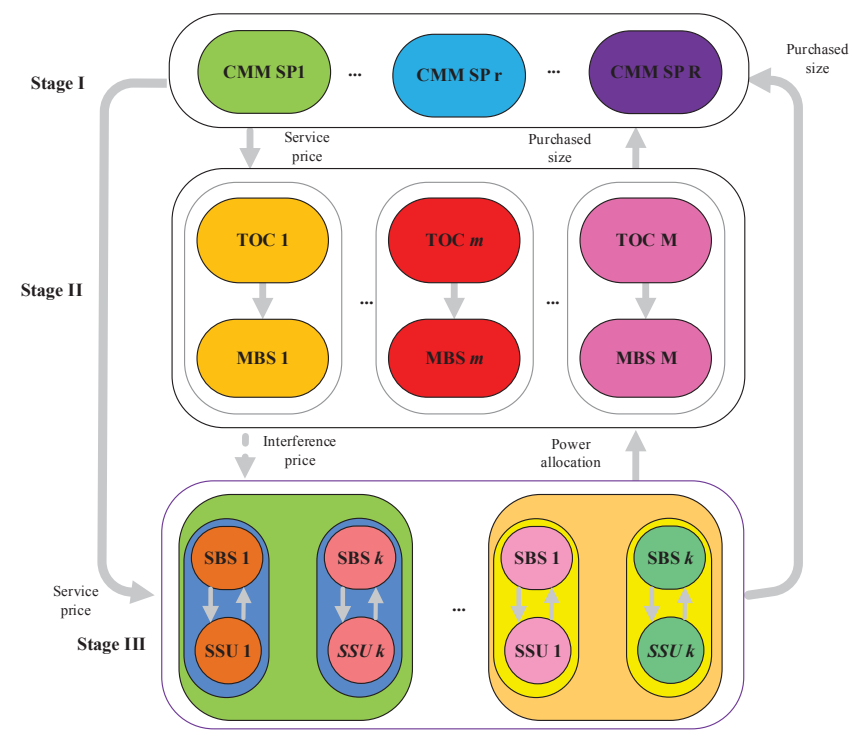

Figure 4.4: Stackelberg game model.

slotted manner, and all the cells are considered to be synchronized perfectly. OFDMA technology is used in this architecture.

\subsubsection{Problem Formulation}

In this chapter, we extend thee model discussed in Chapter 3, which is shown in Fig. 4.4. Each third party CMM SP is a leader that can provide the CMM service $x_{r}$ to the small cells and macrocells via MNaaS business model. All the MUs and SSUs as the followers decide to purchase amount of the CMM service, which is measured in data rate to meet the end-users' high QoS CMM service demands in Stage I. Then in Stage II, we group the TOC and the MBS that is set up by the specific operator as a group to play a leader role to offer an interference price $y$ to the small cells to reduce the interference effect. The group also as the follower decides to purchase the CMM services from which third party SP. In Stage III, the small cells firstly, act as the followers decide to connect which MBS to access the CMM service. Then they 
adjust the power allocation to distribute the CMM service to the SSUs to guarantee the QoS.

CMM SP level game We assume each CMM SP is selfish and independent of gaining the profit as much as possible. Each of their profits depends on its own resource cost and CMM service price, as well as the price offered by the other SP. For an arbitrary SP, the utility function is defined as

$$
U_{r}(x)=\left(x_{r}-c_{r}\right)\left(\sum_{m=1}^{M} s_{m} B_{r m}+\sum_{k=1}^{K} s_{k} B_{r k}\right),
$$

where the price vector $x=\left\{x_{1}, \ldots, x_{r}, \ldots, x_{R}\right\}$ denotes the price offered by the SP, and $c_{r}$ denotes the cost such as the server cost, infrastructure cost, power usage, and networking cost [45]. We assume that the cost of each CMM SP is different from the others. In the function, $s_{m}$ and $s_{k}$ denote the size of the CMM service that the MBS $m$ and SBS $k$ are willing to purchase from the CMM SP $r$. The parameter $B_{r m} \in\{0,1\}$, and $B_{r k} \in\{0,1\}$ denote whether MBS $m$ and SBS $k$ purchase the service from SP $r$ or not respectively, where 1 means yes and 0 means no. The SP needs to find an optimal price to the end-users to maximize its profit, which can be solved by the following problem

$$
\max _{x_{r} \geq c_{r}} U_{r}(x)
$$

MBS and SBS level game We consider the entire HWNs consisting of a set of $M$ MBS and $K$ SBS. Some end-users may have their own special demands (e.g., business, education). In this scenario, we can group those SBSs with the same properties. We suppose that $K$ SBSs are divided into a set of $G=\{1, \ldots, g, \ldots, G\}$ according to their social context. The end-users in the same group has the ability to connect any 
MBS $m$ and it can connect to only one MBS each time.

We assume that TOC and MBS constitute a group. The TOC, as a "broker" purchasing the CMM service with its own MBS, is willing to share those service by allocating portion of wireless resource to the SBSs. We denote the $s_{m}$ as the size of the service resource that MBS is willing to share. Generally, the $s_{m}$ has its boundary: $0 \leqslant s_{m} \leqslant c_{m} \cdot c_{m}$ is the capacity of the MBS. Let $n_{m}$ denote the total number of the SBSs which is connected to the MBS $m$. We assume that all the SBSs connected to the same MBS $m$ are located the identical CMM service: $s_{m} / n_{m}$. Then we define $n_{m}^{g}$ as the number of the SBSs in group $g$ that can choose the specific MBS $m$. We can formulate that $n_{m}=\sum_{g} n_{m}^{g}$. Thus we can also find the size of CMM service for one SBS in group $g$ is that $s_{k}=s_{m} / \sum_{g} n_{m}^{g}$.

In this level of game, the strategy of the SBS is to connect to which group of MBS and TOC. In order to experience a high quality of CMM service, each SBS is eager to purchase the largest size of the CMM service allocation. However, this action may lead the traffic congestion and interference in a specific MBS if it is simultaneously connected by a large number of the SBSs. To address this issue, we propose a dynamic scheme for the MBS to adjust its load. To avoid the mutual interference between the MBS and SBSs, we suppose that the SBSs which are connected to the same MBS will be charged an identical interference price $y_{m}$ as a credit. Once all the SBSs have made their decisions, the strategies are presented as a profile $(\mathbf{s}, \mathbf{y})$, where $\mathbf{s}=\left[s_{1}, \ldots, s_{m}, \ldots, s_{M}\right]^{T}$ and $\mathbf{y}=\left[y_{1}, \ldots, y_{m}, \ldots, y_{M}\right]^{T}$.

We need to consider both the CMM service quality (data rate), and also the infrastructure consumption. To these ends, the MBS needs to limit the interference from the small cells by offering the interference price $y_{m}$, we model the competition among the MBSs as a noncooperative game, and consider the NE as the solution of 
the game. The utility function of the MBS is defined as below,

$$
U_{m}\left(s_{m}, y_{m}\right)=\delta y_{m} \times \sum_{g \in G} n_{m}^{g}(s, y)-\omega_{m} s_{m}
$$

$\delta$ is an equivalent satisfaction per unit price contributing to the entire utility, $y$ is the strategy set, and $\omega_{m}$ is the unit cost for data transmission. $n_{m}^{g}$ is the total number of SBSs in group $g$ connecting to the MBS $m$, which is also defined as the function of $(s, y)$. We also define the capacity of the MBS $m$ below,

$$
c_{m}=W_{B} \log _{2}\left(1+\frac{p_{m} h_{m}^{2}}{\sigma_{m}^{2}+\sum_{k=1}^{n_{k}^{g}} g_{k m}^{2} p_{k}}\right) .
$$

$W$ denotes the bandwidth of each channel, $h_{m}$ denotes the channel gain including the path-loss and the small-scale fading process, $p_{m}$ denotes the transmit power of MBS $m, \sigma_{m}$ is AWGN with zero mean and unit variation, and $g_{k m}$ denotes the channel gain of small cell in the group $g$ including the path-loss and the small scale fading process.

The optimization problem for the MBS can be formulated as,

$$
\max _{0 \leq s_{m} \leq c_{m}, y_{m} \geq 0} U_{m}\left(s_{m}, y_{m}\right)
$$

Then we study the utility function of the SBS $k$. Firstly a SBS is always willing to connect to a MBS. Because all the SBSs in one group can exchange the information about their strategies. If one SBS observes another SBS changes its decision to connect to another MBS for purchasing high quality of CMM service, it will learn this strategy and change its own decision with the hope of achieving a higher utility. Then we can formulate the utility of one SBS which connects to the MBS $m$ in group 
$g$ as,

$$
\pi_{m}^{g}=\varphi\left(\gamma s_{k}\right)-\omega_{k} y_{m}
$$

$\gamma$ is a pre-defined parameter for various service, $\omega_{k}$ is an equivalent satisfaction per unit interference price contributing to the entire utility. $\varphi(\cdot)$ measures the satisfaction of the allocated CMM service. It is supposed to be a concave function of $s_{k}$. Then we can rewrite the utility function as,

$$
\pi_{m}^{g}=\log \left(\gamma \frac{s_{m}}{\sum_{g} n_{m}^{g}}\right)-\omega_{k} y_{m}
$$

Because we have described that the SBS in the same group can learn the strategy with each other. The strategy of one SBS in a population can be replicated by the others. These replications form the evolution in the population. We introduce the replicator dynamics method to solve this problem [59]. In mathematics, the replicator dynamics is a deterministic monotone non-linear and non-innovative game used in evolutionary game theory. In the replicator dynamics scheme, the share of a strategy in the population increases at a level equalling to the difference between the utility of the strategy and the average utility of the population. In this chapter, we let $x^{g}$ as the vector of proportion of the population state. The set can be expressed as $x^{g}=\left[x_{1}^{g}, \ldots, x_{m}^{g}, \ldots x_{M}^{g}\right]$. Note that $\sum_{m=1}^{M} x_{m}^{g}=1$ and $x_{m}^{g}>0$.

Because of the latency, the SBSs in the same group may not receive the up-todate state $x^{g}$. Hence, they have to make their decisions according to the historical information. We consider the delay in the replicator equations, then we can get,

$$
\dot{x}_{m}^{g}(t)=x_{m}^{g}(t)\left(\pi_{m}^{g}(t)-\phi_{m}^{g}(t)\right)
$$


$\phi(\cdot)$ is the function of the average utility. When all the strategies do not change anymore, the evolution is over. By solving $\dot{x}_{m}^{g}(t)$, the evolutionary equilibrium can be obtained. Since the selection of the strategy is zero, no SBS is willing to change its own strategy in the state of evolutionary equilibrium.

We assume that each SBS has only one SSU each time. Therefore, the utility function for an arbitrary SBS $k$ can be defined as below,

$$
U_{k}\left(s_{k}, p_{k}\right)=s_{k}-\mu_{k} x_{r} s_{k} B_{r k}-\lambda_{k} y_{m} g_{k m}^{2} p_{k}
$$

$\mu_{k}$ denotes the weight, which represents the tradeoff between the data rate and CMM service price. $\lambda_{k}$ is tradeoff parameter between the interference fee and transmission power. The optimization problem for and arbitrary SSU $k$ can be formulated as,

$$
\max _{0<s_{k} \leq c_{k}, p_{k} \geq 0} U_{k}\left(s_{k}, p_{k}\right)
$$

$c_{k}$ is the capacity of the SBS $k$,

$$
c_{k}=W_{B} \log _{2}\left(1+\frac{p_{k} h_{k}^{2}}{\sigma_{k}^{2}}\right) .
$$

$h_{k}$ denotes the channel gain between SBS $k$ and SSU $k$ including the path-loss and the small-scale fading process, $p_{k}$ denotes the transmit power of the SBS $k . \sigma_{k}$ is AWGN.

\subsection{Stackelberg Game Analysis}

In this section, we analyze the proposed Stackelberg game. Then we obtain the equilibrium of this game. Based on the description of the system, we know that each 
strategy of the stage will affect the strategies of the other level. Hence, we choose backward induction method to solve this game. We consider a specific scenario of HWNs model with two TOC and MBS groups, i.e., the set $M=\{1,2\}$.

\subsubsection{SBS Level Game Analysis}

For maximizing the utility function for SSU, each SSU will choose a proper CMM SP to purchase the CMM service according to the price $x_{r}$. We can obtain the function as,

$$
U_{k}\left(s_{k}\right)=\left(1-\mu_{k} x_{r} B_{r k}\right) s_{k}-\lambda_{k} y_{m} g_{k m}^{2} p_{k}
$$

We consider the solution of this utility function into two cases.

When $1-\mu_{k} x_{r} B_{r k}>0, U_{k}\left(s_{k}\right)$ is a monotonic increasing function. In order to maximize $U_{k}$, we can use the decomposition theory. We decompose the optimization problem into two sub optimization problems, fix $s_{k}$ then make $p_{k}^{*}$ to be the optimum. Then we can get,

$$
\frac{\partial^{2} U_{k}}{\partial p_{k}{ }^{2}}=-\frac{W_{B} h_{k}{ }^{4}\left(1-\mu_{k} x_{r} B_{r k}\right)}{\ln 2\left(p_{k} h_{k}^{2}+\sigma_{k}{ }^{2}\right)}<0 .
$$

Therefore, this utility function is a concave function of $p_{k}$. After the calculation, we obtain the optimal power allocation strategy $p_{k}^{*}$ as below,

$$
p_{k}^{*}=\left[\frac{W_{B}\left(1-\mu_{k} x_{r} B_{r k}\right)}{\ln 2 \lambda_{k} y_{m} g_{k m}^{2}}-\frac{\sigma_{k}^{2}}{h_{k}^{2}}\right]^{+},
$$

When $1-\mu_{k} x_{r} B_{r k}<0, U_{k}\left(s_{k}\right)$ is a monotonic decreasing function. Hence, when $s_{k}^{*}=0$, the function will the the maximum value, but this situation makes no sense in the real environments. When an SSU applies for service from a CMM SP, its data 
rate should retain above zero.

In this level, because we have demonstrated that the evolutionary game can reach its evolutionary equilibrium when $x_{m}^{g}(t)=0$ in Section 4.1.3. The stop condition is that the group member choosing different MBSs will obtain identical utility since no SBS can find the others in the same group can get higher utility. Therefore, we can get the evolutionary equilibrium by solving the following equation,

$$
\pi_{m}^{g}=\pi_{n \neq m}^{g}, \forall g \in G
$$

Then we can obtain the equation in this particular case:

$$
\log \left(\gamma \frac{s_{1}}{\sum_{g} n_{1}^{g}}\right)-\omega_{k} y_{1}=\log \left(\gamma \frac{s_{2}}{\sum_{g} n_{2}^{g}}\right)-\omega_{k} y_{2}
$$

The left side represents the utility of SBSs choosing the MBS 1 and the right side

represents the utility of SBSs choosing the MBS 2. Substituting the equation $n_{m}^{g}=$ $x_{m}^{g} n^{g}$ into (4.16). Then we can obtain,

$$
\log \left(\gamma \frac{s_{1}}{\sum_{g} x_{1}^{g} n^{g}}\right)-\omega_{k} y_{1}=\log \left(\gamma \frac{s_{2}}{\sum_{g}\left(1-x_{1}^{g}\right) n^{g}}\right)-\omega_{k} y_{2}
$$

Then we can get the evolutionary equilibrium below,

$$
\sum_{g} x_{1}^{g} n^{g}=\frac{\sum_{g} n^{g}}{\frac{s_{2}}{s_{1}} \cdot e^{\omega_{k}\left(y_{1}-y_{2}\right)}+1} .
$$

\subsubsection{MBS and TOC Level Game Analysis}

Based on the decision of the SBSs, the group of MBS and TOC is willing to adjust its strategy to achieve a higher utility. We assume that the CMM service of both MBS 1 and 2 are fixed. Then we know that the interference price $y_{m}$ is a single strategy. 
Therefore, the utility function of MBS $m$ can be rewritten below,

$$
U_{m}=\delta y_{m} \times\left(\sum_{g} x_{m}^{g} n^{g}\right)-\omega_{m} s_{m},
$$

Then, we substitute the (4.18) into (4.19),

$$
U_{m}=\delta y_{m} \times\left(\frac{\sum_{g} n^{g}}{\frac{s_{m}}{s_{n}} \cdot e^{\omega_{k}\left(y_{m}-y_{n}\right)}+1}\right)-\omega_{m} s_{m} .
$$

The first order derivative of this utility function is shown below,

$$
\begin{aligned}
\frac{\partial U_{m}}{\partial y_{m}}= & \delta\left(\sum_{g} n^{g}\right) s_{m} \\
& \times \frac{s_{n} \cdot e^{\omega_{k}\left(y_{m}-y_{n}\right)}+s_{m}-s_{n} \cdot e^{\omega_{k}\left(y_{m}-y_{n}\right)} \omega_{k} y_{m}}{\left[s_{n} \cdot e^{\omega_{k}\left(y_{m}-y_{n}\right)}+s_{m}\right]^{2}} .
\end{aligned}
$$

By setting the $\frac{\partial U_{m}}{\partial y_{m}}=0$. We can get,

$$
y_{m}^{*}=\frac{s_{m}}{\omega_{k} s_{n}} \cdot e^{\omega_{k}\left(y_{m}-y_{n}\right)}+\frac{1}{\omega_{k}} .
$$

We introduce the Lambert-W function [60], then we can rewrite the $y_{m}$ function,

$$
y_{m}^{*}=\left[\frac{1}{\omega_{k}} W\left(\frac{s_{m}}{s_{n}} \cdot e^{\left(\omega_{k} y_{n}-1\right)}\right)+\frac{1}{\omega_{k}}\right]^{+} .
$$

We know that the utility of function is monotonic increasing about $s_{m}$, when $s_{m}$ chooses the maximum value, the function will also achieve the maximum value. Then 
we can obtain the optimum, when $s_{m}=c_{m}$,

$$
\begin{aligned}
& s_{m}^{*}= \\
& {\left[W_{B} \log _{2}\left(1+\frac{p_{m} h_{m}^{2}}{\sigma_{m}^{2}+\sum_{k=1}^{n_{i}^{g}} g_{k m}^{2}\left(\frac{W\left(1-\mu_{k} x_{r} B_{r k}\right)}{\ln 2 \lambda_{k} y_{m}^{*} g_{k m}^{2}}-\frac{\sigma_{k}^{2}}{h_{k}^{2}}\right)}\right)\right]^{+} .}
\end{aligned}
$$

\section{Nash Equilibrium Analysis}

So far, in (4.23), the utility of the interference price of one TOC and MBS group increases with the rival's higher price. Then we can get the existence of the NE depending on the proof of the supermodular game.

Proof. From [61], we conclude that the supermodular has the following three characteristics.

1. A strategy is a complete lattice.

2. An utility exhibits strictly increasing differences in all sets.

3. An utility is supermodular in its own strategy (given the others).

For any player $m$ in the game, its interference price $y_{m} \in(0, \infty)$. Hence, we can verify the first characteristic very easily. To prove the other characteristics 2 and 3 , we can compute the $\frac{\partial^{2} U_{m}}{\partial y_{m} \partial y_{n}}$ as below,

$$
\frac{\partial^{2} U_{m}}{\partial y_{m} \partial y_{n}}=\frac{W(Z)}{1+W(Z)}
$$

where we define that $Z=\frac{s_{m}}{s_{n}} \cdot e^{\left(\omega_{k} y_{m}-1\right)}$. From the features of the Lambert-W function, we know that $Z=W(Z) e^{W(Z)}$. If $Z>0$, then $W(Z)>0$ and we obtain that 
$\frac{\partial^{2} U_{m}}{\partial y_{m} \partial y_{n}}>0$. Thus the non-cooperative game is a supermodular game, which exists an NE.

Secondly, we will discuss the uniqueness of the NE.

Proof. To approve the uniqueness of the NE, we apply Lambert-W function again. The self-mapping function can be shown as below,

$$
\begin{aligned}
y_{m} & =T_{m}\left(y_{m}\right)=\rho_{m}\left(\rho_{n}\left(y_{m}\right)\right) \\
& =\frac{1}{\omega_{k}}\left(1+W\left(\frac{e^{\omega_{k} y_{n}-1}}{W\left(\frac{s_{m}}{s_{n}} \cdot e^{\omega_{k} y_{n}-1}\right)}\right)\right) .
\end{aligned}
$$

We recall the contraction mapping principle. The contraction maps have unique fixed points. If we prove the mapping function is contraction. Then the uniqueness of the NE is proved. From (4.26), we obtain the Jacobian matrix as below,

$$
J=\left(\begin{array}{cc}
\frac{\partial^{2} T_{m}}{\partial y_{m}^{2}} & \frac{\partial^{2} T_{m}}{\partial y_{m} \partial y_{n}} \\
\frac{\partial^{2} T_{n}}{\partial y_{m} \partial y_{n}} & \frac{\partial^{2} T_{n}}{\partial y_{n}^{2}}
\end{array}\right) .
$$

Then we obtain the absolute eigenvalue of (4.27) as,

$$
\eta=\sqrt{\frac{\partial T_{m}}{\partial y_{n}} \times \frac{\partial T_{n}}{\partial y_{m}}} .
$$

From (4.25), we know that $\frac{\partial^{2} U_{m}}{\partial y_{m} \partial y_{n}}=\frac{\partial T_{m}}{\partial y_{n}}<1$. Hence, $\eta<1$, then the uniqueness of $\mathrm{NE}$ is proved. 


\subsubsection{CMM SPs Level Game Analysis}

The aim of the CMM SPs is to take the service prices as their strategic variables to maximize their own profits. In this chapter, we choose the homogenous Bertrand method to solve the game. The CMM SPs offer the service to the end-users, and we assume the service is commodity. Hence, the scenario is homogeneous. The profits of an arbitrary CMM SP $r$ depends not only on the CMM service price $x_{r}$, and the cost of $c_{r}$ but also on the CMM service prices $x_{-r}=\left(x_{1}, x_{2}, \ldots, x_{r-1}, x_{r+1}, \ldots, x_{R}\right)$ offered by the other CMM SPs. In this level, each CMM SP decides its own strategy independently and simultaneously. And the cloud computing platform with the lowest service price will occupy the entire service market. To gain a higher market profit, each CMM SP is willing to reduce its service price until hitting the bottom with zero profit. As shown in Section 4.1.3, we know that the set of the game players in this level is $\boldsymbol{R}=\{1, \ldots, r, \ldots, R\}$, and the strategy set is $\mathbf{x}_{r}=\left\{x_{1}, \ldots, x_{r}, \ldots, x_{R}\right\}$. The NE of this problem gives that neither of the player $r$ can increase their utility function $U_{r}$ by unilaterally changing the price. Without loss of generality, we sort the cost set $\mathbf{c}_{r}$ in an ascending order $c 1<\ldots<c_{r}<\ldots<c_{R}$.

Proposition 4.2.1. The NE of the proposed homogeneous Bertrand game with multiple SPs is shown below,

$$
x^{*}=\left\{x_{1}^{*}, c_{2}, c_{3}, \ldots, c_{\mathcal{R}}\right\} .
$$

$x_{1}^{*}$ denotes the price strategy of the first cloud platform provider at NE. It can be formulated as,

$$
x_{1}^{*}=\arg \max _{c_{1} \leq x_{1}<c_{2}} U_{1}^{M}\left(x_{1}\right)
$$

$U_{1}^{M}$ is the utility function of the provider 1 when it supplies the whole market, 
shown as (4.31). We define that $I_{m}=\sigma_{m}^{2}+\sum_{k=1}^{K} g_{k m}^{2}\left[\frac{W_{B}\left(1-\mu_{k} x_{r} B_{r k}\right)}{\ln 2 \lambda_{k} y_{m} g_{k m}^{2}}-\frac{\sigma_{k}^{2}}{h_{k}^{2}}\right]^{+}$.

$$
\begin{aligned}
& U_{1}^{M}=\left(x_{1}-c_{1}\right)\left(\sum_{m=1}^{M} B_{r m}\left[W_{B} \log _{2}\left(1+\frac{p_{m} h_{m}^{2}}{I_{m}}\right)\right]^{+}\right) \\
& +\left(x_{1}-c_{1}\right)\left(\sum_{k=1}^{K} B_{r k}\left[W_{B} \log _{2}\left(\frac{h_{k}^{2} W_{B}\left(1-\mu_{k} x_{1} B_{r k}\right)}{\ln 2 \lambda_{k} y g_{k m}^{2} \sigma_{k}^{2}}\right)\right]^{+}\right) .
\end{aligned}
$$

Proof. We assume in the Bertrand condition, there are only two CMM SPs, the SP 1 and the SP 2 in the competition. The costs of these two players are $c_{1}$ and $c_{2}$, and $c_{1}<c_{2}$. Based on the Bertrand method, both SPs have the incentives to reduce their CMM service prices down to their own cost margin to capture the whole market and undercut the other to double its profit. That is to say, either player is willing to raise its CMM service price over the cost margin, then it will gain nothing. Since all the end-users would purchase the CMM service from the SP who is still setting a competitive price. If a SP has a minor average cost, it can take all the business. Therefore, the SP 1 has the incentive to make its CMM service price between the set of $\left[c_{1}, c_{2}\right)$ to maximize its own profit.

Though it is irrational to set the CMM service price below the marginal cost, the two CMM SPs would make the prices lower than their own monopoly prices. If the SP 2 presents a high enough price, the SP 1 can definitely ignore the affection of SP 2 and set its price by the optimal monopoly price.

Due to the piecewise property about $x_{1}$, to maximize the utility function $U_{1}^{M}$, for the set $\mathbf{M}$ and $\mathbf{K}$. We let,

$$
F_{m}=\frac{W_{B}-\ln 2 \lambda_{k} y_{m}\left[\frac{1}{K}\left(p_{m} h_{m}^{2}-\sigma_{m}^{2}\right)+g_{k m}^{2} \frac{\sigma_{k}^{2}}{h_{k}^{2}}\right]}{W_{B} \mu_{k} B_{r k}},
$$




$$
F_{k}=\frac{W_{B} h_{k}^{2}-\ln 2 \lambda_{k} y_{m} g_{k m}^{2} \sigma_{k}^{2}}{W_{B} h_{k}^{2} \mu_{k} B_{r k}}
$$

Then sort all $F_{1}, F_{2}, \ldots, F_{m}$, and $F_{k}$ in an ascending order $F_{1} \leq F_{2} \leq \ldots F_{m} \leq F_{k}$, without loss of generality. Hence we can get $K$ intervals, $\left[0, F_{1}\right),\left(N_{2}, F_{3}\right), \ldots,\left(F_{m}, F_{k}\right)$. By piecewise differentiating of the utility function in the first interval, we assume that $0<x_{1}<F_{1}$, the second order derivative of $U_{1}^{M}\left(x_{1}\right)$ is shown as (4.34).

$$
\begin{aligned}
& \frac{\partial^{2} U_{1}^{M}\left(x_{1}\right)}{\partial x_{1}^{2}}= \\
& 2\left\{\sum_{k=1}^{K} \frac{W_{B}^{2} \mu_{k} B_{r k} B_{r m} p_{m} h_{m}^{2}}{(\ln 2)^{2} \lambda_{k} y_{m} I_{m}\left(p_{m} h_{m}^{2}+I_{m}\right)}-\sum_{k=1}^{K} \frac{B_{r k}^{2} W_{B} \mu_{k}}{\ln 2\left(1-\mu_{k} x_{1} B_{r k}\right)}\right\} \\
& +\left(x_{1}-c_{1}\right)\left\{\sum_{k=1}^{K} \frac{W_{B}^{3} \mu_{k}^{2} B_{r k}^{2} B_{r m} p_{m} h_{m}^{2}\left(p_{m} h_{m}^{2}+2 I_{m}\right)}{(\ln 2)^{3} \lambda_{k} y_{m} I_{m}^{2}\left(p_{m} h_{m}^{2}+I_{m}\right)^{2}}-\sum_{k=1}^{K} \frac{W_{B} B_{r k}^{3} \mu_{k}^{2}}{\ln 2\left(1-\mu_{k} x_{1} B_{r k}\right)^{2}}\right\} .
\end{aligned}
$$

We can substitute the $I_{m}$ into (4.34), then we know that $\frac{\partial^{2} U_{1}^{M}\left(x_{1}\right)}{\partial x_{1}{ }^{2}}$ is less than 0 that means the $\frac{\partial U_{1}^{M}\left(x_{1}\right)}{\partial x_{1}}$ is a monotonic decreasing function of $x_{1}$. After calculation, we formulate $\frac{\partial U_{1}^{M}\left(x_{1}\right)}{\partial x_{1}}$ as $(4.35)$.

$$
\begin{aligned}
& \frac{\partial U_{1}^{M}\left(x_{1}\right)}{\partial x_{1}}= \\
& \sum_{m=1}^{M} B_{r m} W_{B} \log _{2}\left(1+\frac{p_{m} h_{m}^{2}}{I_{m}}\right)+\sum_{k=1}^{K} B_{r k} W_{B} \log _{2} \frac{W_{B} h_{k}^{2}\left(1-\mu_{k} x_{1} B_{r k}\right)}{\ln 2 \lambda_{k} y_{m} g_{k m}^{2} \sigma_{k}^{2}} \\
& +\left(x_{1}-c_{1}\right)\left\{\sum_{k=1}^{K} \frac{W_{B}^{2} \mu_{k} B_{r k} B_{r m} p_{m} h_{m}^{2}}{(\ln 2)^{2} \lambda_{k} y_{m} I_{m}\left(p_{m} h_{m}^{2}+I_{m}\right)}-\sum_{k=1}^{K} \frac{B_{r k}^{2} W_{B} \mu_{k}}{\ln 2\left(1-\mu_{k} x_{1} B_{r k}\right)}\right\} .
\end{aligned}
$$


We have, $\lim _{x_{1} \rightarrow 0} \frac{\partial U_{1}^{M}\left(x_{1}\right)}{\partial x_{1}}>0$. When $x \rightarrow F_{1}$, we get two cases: $\lim _{x \rightarrow F_{1}} \frac{\partial U_{1}^{M}\left(x_{1}\right)}{\partial x_{1}} \geq 0$ and $\lim _{x \rightarrow F_{1}} \frac{\partial U_{1}^{M}\left(x_{1}\right)}{\partial x_{1}}<0$. For the first case, the utility $U_{1}^{M}$ is strictly monotonic increasing about $x_{1}$ at the initial interval $\left[0, F_{1}\right)$. Therefore, for the second case, we know that the utility function $U_{1}^{M}$ firstly climbs up with $x_{1}$. After reaching the optimal point

it drops down with $x_{1}$. Hence, the utility function $U_{1}^{M}$ is a concave function at the first interval, and it is easily to prove the utility function $U_{1}^{M}$ is a concave function at the other intervals. That is to say, for $x_{1}<F_{k}$, the utility function is a concave function without the most $K$ non-differentiable points at $F_{1}, F_{2}, \ldots, F_{m}$, and $F_{k}$. We can solve $x_{1}$ at each interval by many methods (e.g., the binary search algorithm and the gradient-based algorithm).

In this chapter, it is important to investigate the existence and uniqueness of the NE of Stackelberg game. In the duopoly case the convexity of the follower's reaction function is essential for uniqueness of the NE. Hences, we will prove our Stackelberg game model existing an unique equilibrium.

Theorem 4.1. The unique NE exists in the proposed Stackelberg game.

Proof. Because we have already proven that each level exists a perfect equilibrium in a NE, then the NE of the proposed Stackelberg game model exists. The perfect equilibrium of the sub-game in each level is unique. Hence, the total Stackelberg NE is unique.

\subsection{Service Allocation Algorithms}

In this section we will present algorithms for each level of the game to converge their evolutionary equilibrium. 


\subsubsection{Evaluative Protocols for SBSs}

We present an iteration algorithm for SBS level game based on the replicator dynamics. We assume that each SBS in the same group can exchange the utility information with other. The SBS can decides its own strategy according to the rival's with some possibility. When all the SBSs in the same group reach the same utility, the evolution is complete. We describe the specific procedure as follows.

1. We assume each SBS can only connect to one TOC and MBS group at one time.

2. From (4.7), the utility is calculated with the size of CMM service and the interference price charged by TOC and MBS group.

3. We calculate the average utility of each group as,

$$
\phi^{g}(t)=\frac{\pi_{m}^{g}(t) \cdot \sum_{m} n_{m}^{g}(t)}{n^{g}(t)} .
$$

If the average utility is greater than its own utility, the SBS can change its strategy to the other MBS and TOC group with the possibility as,

$$
P(t)=\frac{\phi^{g}(t)-\pi^{g}(t)}{\pi^{g}(t)} .
$$

4. Repeat step 2 and 3.

Then we can form the algorithm for this level game.

\subsubsection{Protocols for TOC and MBS Group}

We design an algorithm for the TOC and MBS group to update the size of the CMM service and interference price that they can provide. The specific CMM service update 


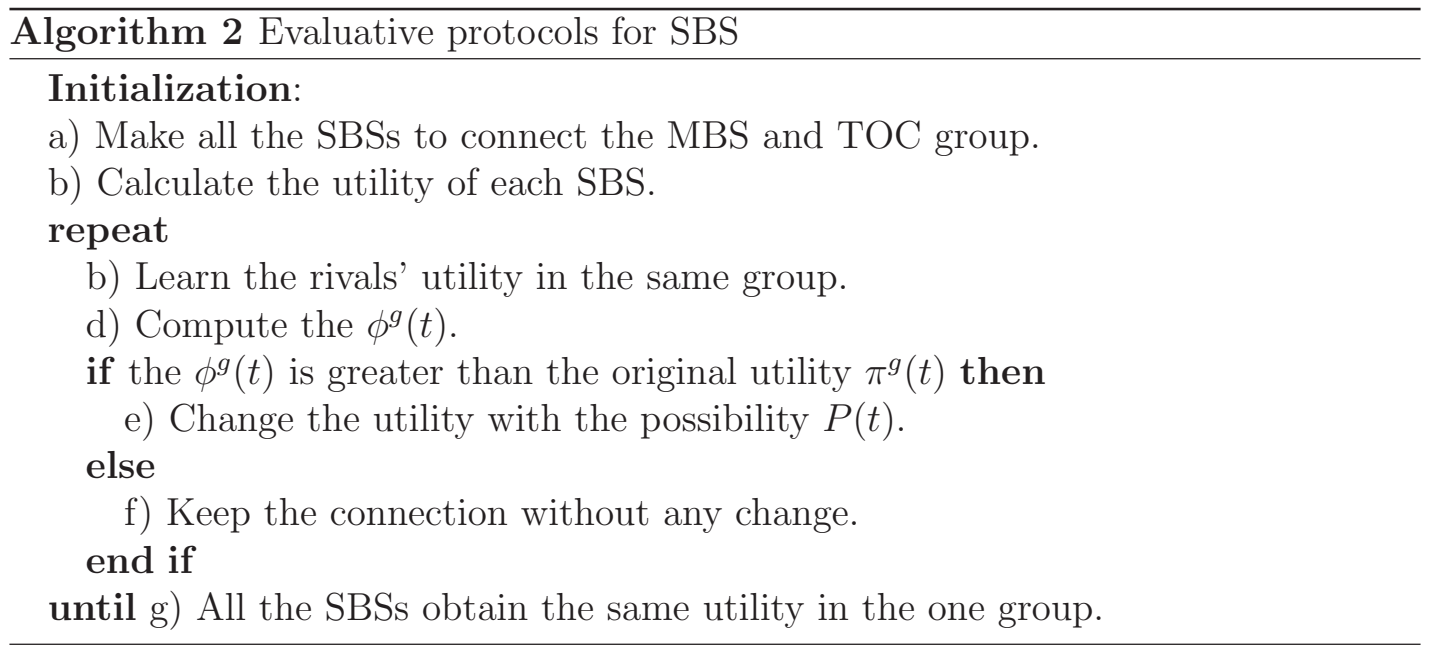

scheme is as following,

$$
s_{m \tau}(t+1)=s_{m \tau}(t)+\rho_{s}\left[U_{m}(t)-U_{m}(t-1)\right] .
$$

The scheme for the interference is,

$$
y_{m \tau}(t+1)=y_{m \tau}(t)+\rho_{y}\left[U_{m}(t)-U_{m}(t-1)\right] .
$$

After updating the strategies, the MBS and TOC group broadcast its refreshed decisions to the SBSs. We assume that SBS can get the new information immediately without delay. Then the SBSs adjust their own strategies according to the algorithm 2. The algorithm 3 shows the execution of the MBS and TOC group.

\subsubsection{CMM Service Allocation Iteration Algorithm}

We introduce the backward induction to solve the entire problem, then we can get the NE of the Stackelberg game and present a CMM service iteration algorithm.

In the above algorithm, we can define the other CMM SPs' strategies as $x_{-r}=$ 

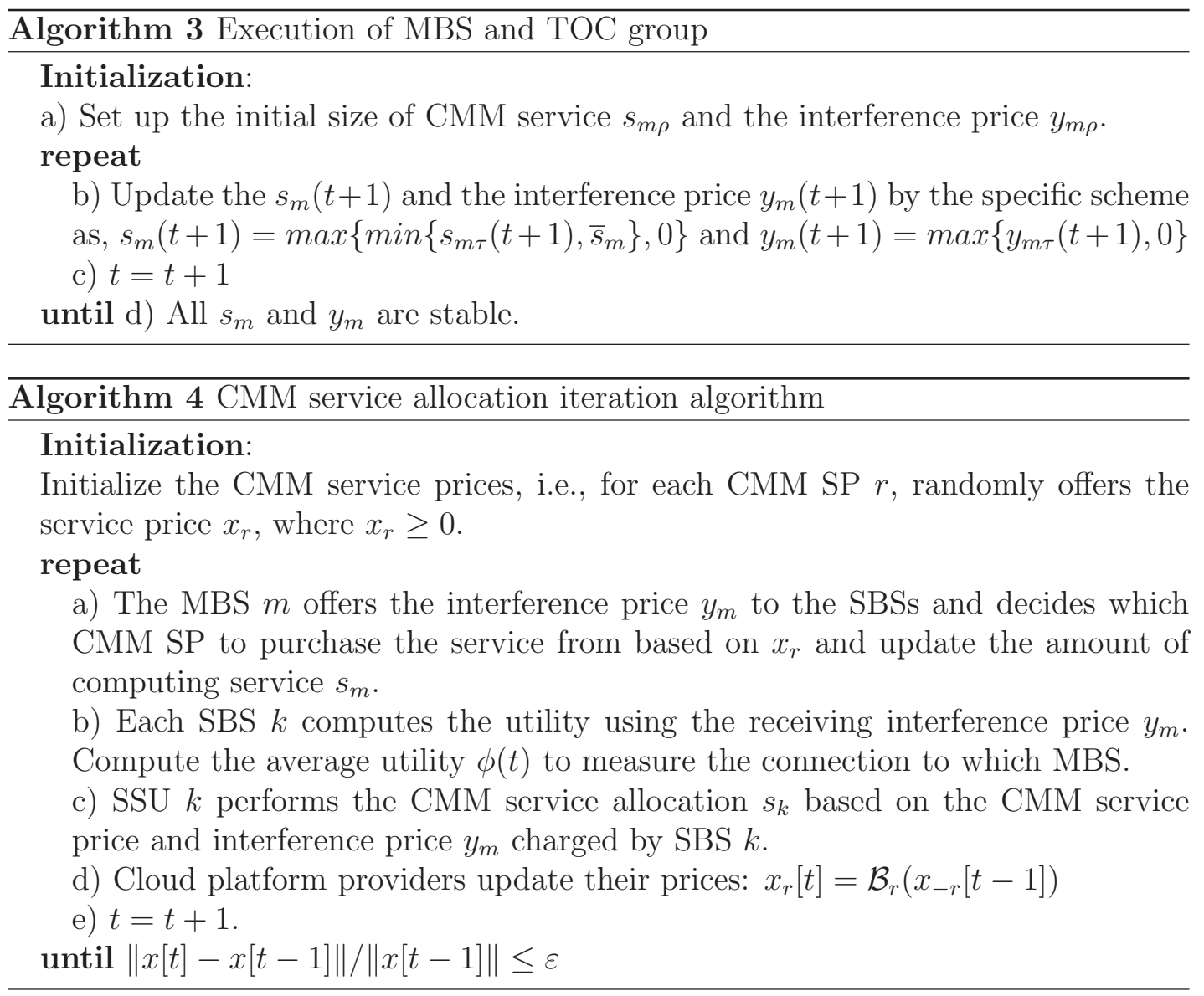

$\left(x_{1}, \ldots, x_{r-1}, x_{r+1}, \ldots, x_{R}\right)$. When $x_{-r}$ is given at iteration step $t-1$, we can present the best response function $\mathcal{B}_{r}\left(\mathbf{x}_{-r}[t-1]\right)$ of CMM service price $x_{r}$ at the iteration step $t$ to maximize its total revenue. The stop condition is $\|x[t]-x[t-1]\| /\|x[t-1]\| \leq \varepsilon$. In the proposed algorithm, the MBS decides the interference price $y_{m}$ offering to SBSs and the amount of service purchased from the CMM SPs based on the service price $x_{r}$. The SBS then decides to connect which MBS. The SSU pays the service price $x_{r}$ and interference price $y_{m}$. The algorithm will stop until the service price $x_{r}$ converge. 


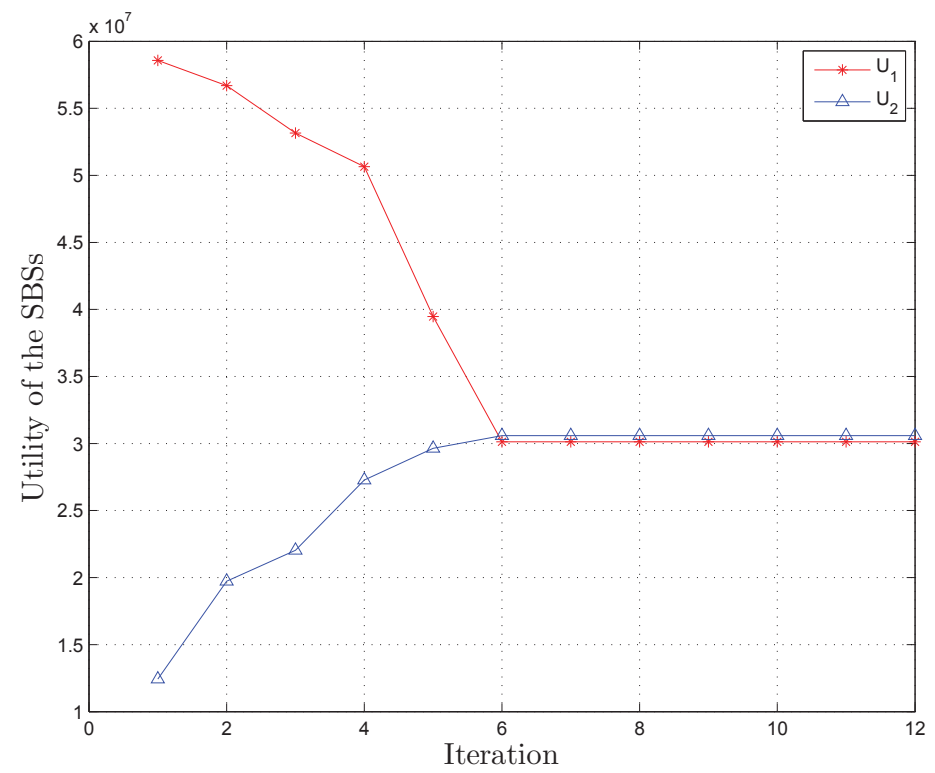

Figure 4.5: Convergence of the evolution among SBSs to the equilibrium.

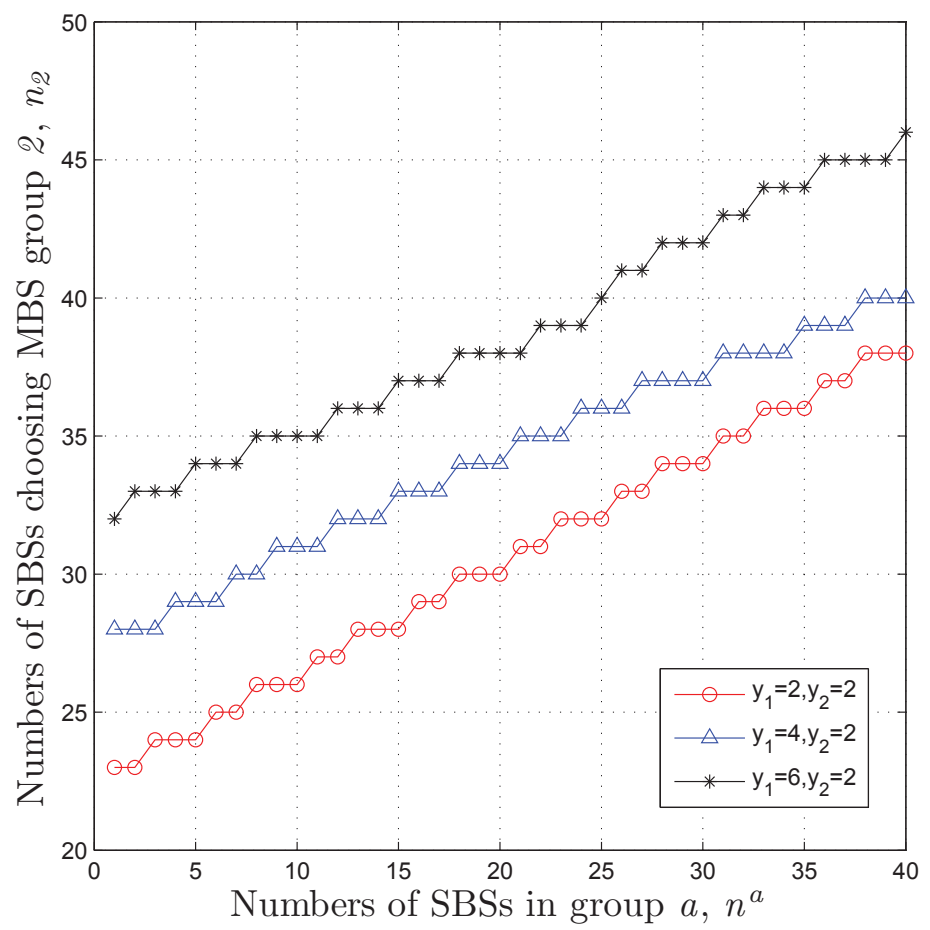

Figure 4.6: Evolutionary equilibrium with the different SBSs group number and the variable interference price. 


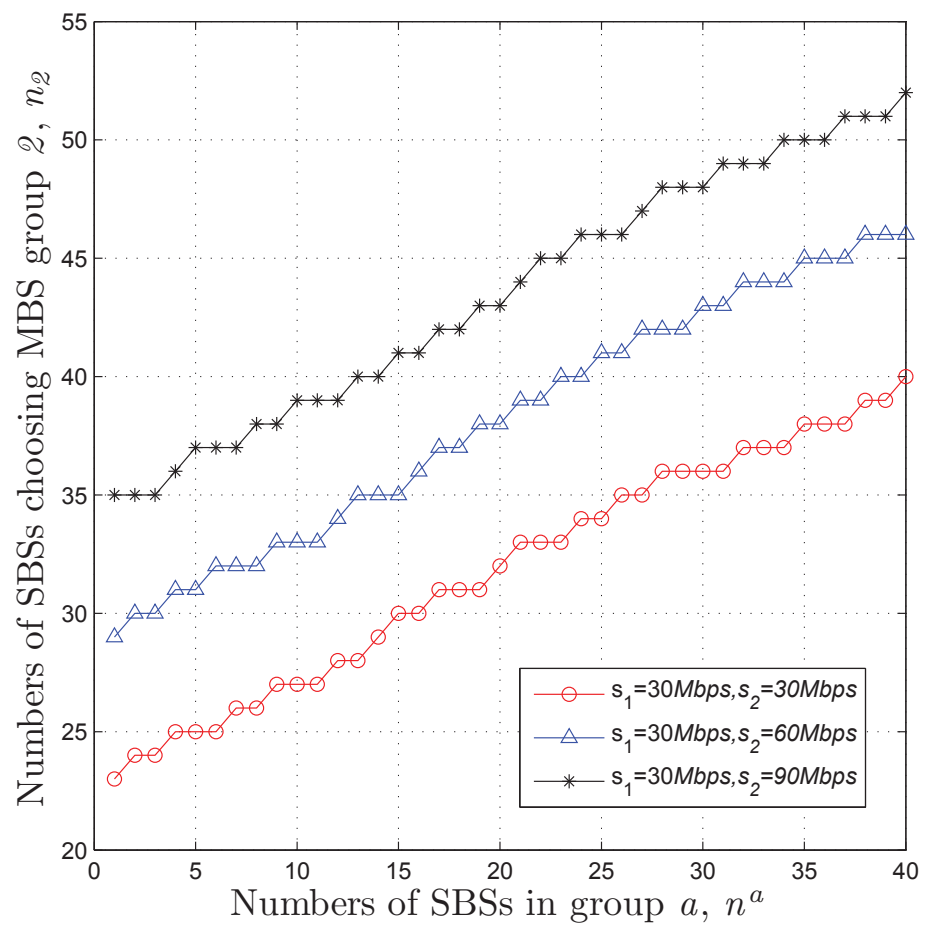

Figure 4.7: Evolutionary equilibrium with the different SBSs group number and the variable CMM service size.

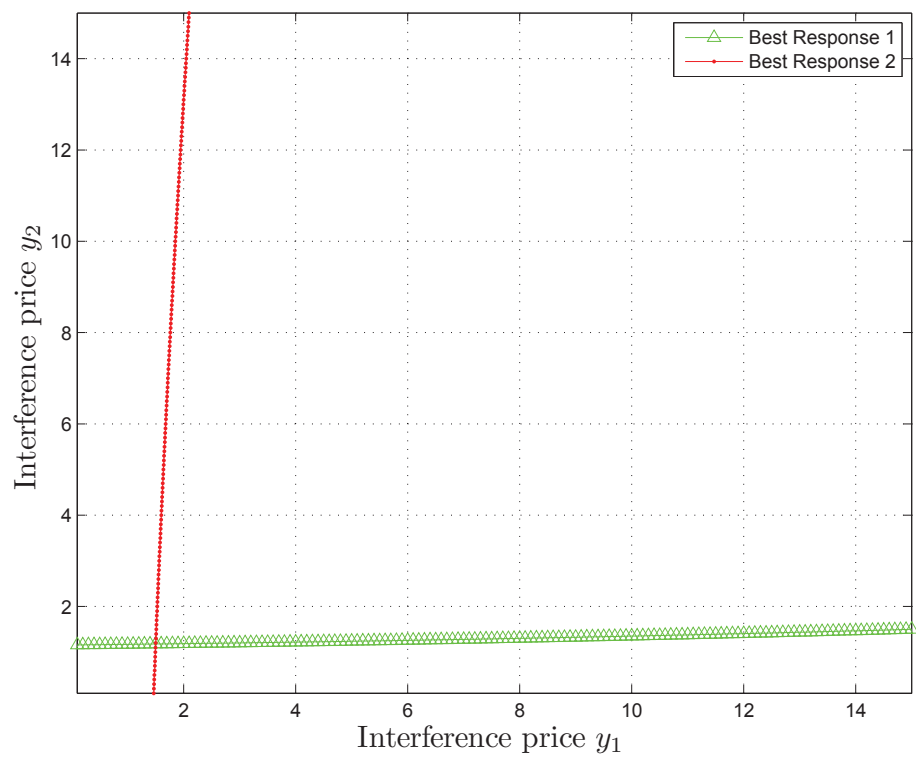

Figure 4.8: Best response for interference prices. 


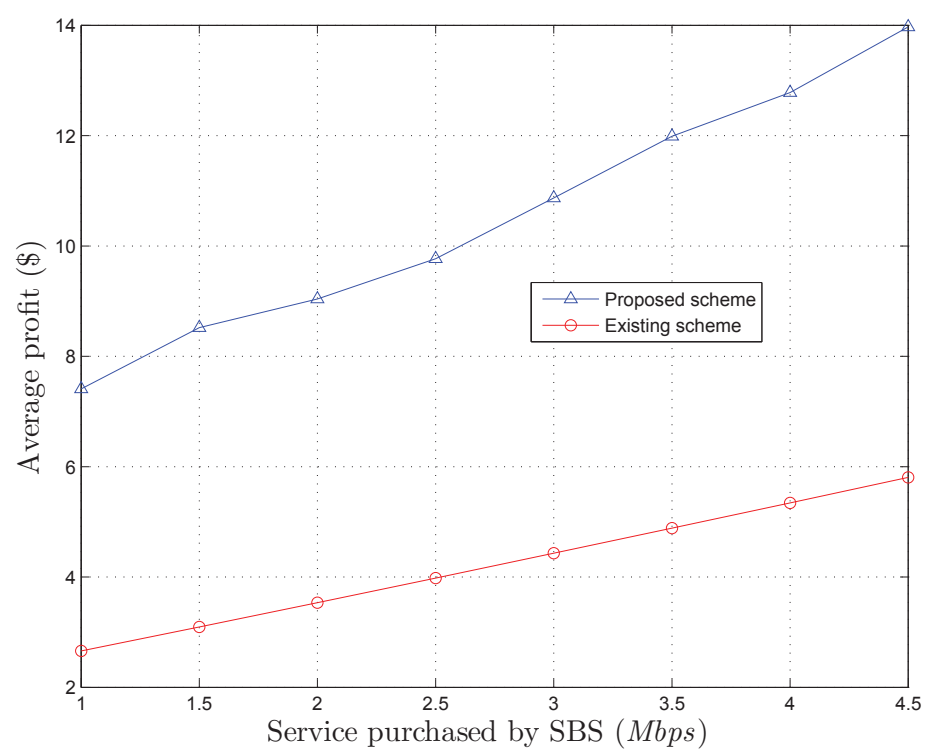

Figure 4.9: The average profit compares with an existing scheme.

\subsection{Simulation Results and Discussions}

In this section, we evaluate the performance of the proposed scheme by the computer simulations. The main system parameters in the HWNs are adopted from the $3 \mathrm{GPP}$ [44]. In these simulations, we assume there are 2 CMM SPs, 2 groups of telecom operators and MBSs, and 2 SBSs groups. The initial number of the SBSs in each group is 25. For the utility function, we set $\omega_{m}=1$ for the MBS and $\gamma=100$, $\omega_{k}=0.5$ for the SBS. The SBSs are located in the marcocell randomly and sparsely with each other at $50 \mathrm{~m}$ to $150 \mathrm{~m}$ far frome the MBS. We set the path loss between SSU and MBS as $15.3+37.6 \log _{10}\left(D_{m s}+L_{o w}\right)$ and the path loss between SSU and SBS as $46.86+20 \log _{10}\left(D_{s s}+L_{i w}\right)$, where $D_{m s}$ is the distance between the MBS and the SSU and $D_{s s}$ is the distance between the SBS and the SSU. $L_{o w}$ means the penetration loss of exterior wall, and $L_{i w}$ means the penetration loss of the interior wall. They are set as $20 d B$ and $10 d B$, respectively. We can also find the parameters of the small scale and shadow fading in the [44]. The transmission bandwidth $W_{B}$ is $5 \mathrm{MHz}$ in the 
MBS and the max transmission power is $46 \mathrm{dBm}$ from [44]. The general parameters are set as, $\mu_{k}=0.05, \lambda_{k}=1, \alpha=0.03, \beta=10, B_{r k}$ and $B_{r m}=1, \rho_{s}=1, \rho_{y}=0.05$.

Firstly we consider the replicator dynamics method to show the evolutionary behavior of the SBSs. We define the two TOC and MBS groups purchase the initial size of the CMM service from the CMM SPs are $s_{1}=s_{2}=30 M b p s$. The two TOC and MBS offers the initial prices as $y_{1}=0.4$ and $y_{2}=0.6$. Fig. 4.5 shows the convergence of the evolutionary behavior when the initial population state is $x_{1}^{a}=0.6$, and $x_{1}^{b}=0.4$. The proposed system takes six steps to reach the equilibrium state. From the system, we know that in the state of the equilibrium, the utility of all the SBSs in both groups are nearly identical.

Fig. 4.6 represents the evolutionary equilibrium with the different SBS group numbers $n_{a}$ and the variable interference price $y_{2}$. We assume that the sizes of the CMM service shared by the two MBS and TOC group both are 30Mbps. The number of SBSs in group $b$ is fixed as 30. We can obtain that the number of SBSs choosing the MBS and TOC group 2 increases with the number of SBSs in group a goes higher. When the interference price offered by the MBS and TOC group 1 increases, the SBSs try to connect the rival with the lower interference price.

Fig. 4.7 indicates that the evolutionary equilibrium with the different SBS group numbers $n_{a}$ and the variable CMM service size $s_{2}$. We set the interference prices offered by the MBS and TOC group are 2. The curves show that the number of SBSs choosing the MBS and TOC group 2 increases with the number in group $a$ have more SBSs. While the MBS and TOC group 2 offers more CMM service, there will be more SBSs connect to the group 2.

Fig. 4.8 gives the best response for the two SBS group $a$ and $b$. We set the CMM service size purchased by group $a$ and $b$ are 30Mbps and 40Mbps respectively. From the figures, we can get that both best response for interference prices $y_{1}$ and 
$y_{2}$ are increasing in the non-cooperative game. There is only one intersection point $(1.20,1.52)$ of the two curves. Hence, we prove that NE is unique in the second of the entire game.

Finally, the average profit for our proposed scheme compared with an existing

scheme. In the existing scheme, it cannot consider the scenario with multiple groups of TOCs and MBSs, as well as ignore the replicator dynamics method. The transmission rate is controlled between 1 and $4.5 \mathrm{Mbps}$. We recalculate the profits of the CMM SPs 1000 times at each 0.5Mbps interval to obtain the average profits. In Fig. 4.9, we compare the average profits of proposed scheme and the existing scheme described in Chapter 3, based on the size of service purchased by one SBS. We can point out that the two schemes both have the growing trends with the increasing size of the CMM service purchased by the SBS. Simulation results show that the proposed scheme can gain more profits than the existing one with the same size of CMM service.

\subsection{Chapter Summary}

In this chapter, we have studied the issues of jointly considering the operations of dynamic clouds and wireless networks in a mobile cloud computing environment. We extended the system model discussed in the previous chapter to include CMM SPs, TOC and HWNs with small cells. We formulated the problems of determining the CMM service price decision, wireless power allocation, and interference management as a three-level Stackelberg game. We also introduced an interference price to handle the interference effects between the macro cells and small cells. Furthermore, we adopted the replicator dynamics method to solve the evolutionary game between the groups of SBSs. At the CMM SPs' level, we proposed a homogeneous Bertrand game with multiple CMM SPs and used a backward induction method to solve the whole model. Finally, we presented an iteration algorithm to obtain the equilibrium of the 
Stackelberg game. Simulation results have been presented to show the performance of our proposed scheme. 


\section{Chapter 5}

\section{Conclusions and Future Work}

\subsection{Conclusions}

In this thesis, we have studied the issues that arise when jointly considering the operations of clouds and wireless networks in a mobile cloud computing environment. We proposed a mobile cloud computing system combined with heterogeneous wireless networks. We consider the pricing information in the cloud, based on how the power allocation and interference management in wireless networks are performed. We adopted the Stackelberg game theoretic approach to formulate the problems of determining the cloud mobile media service price decision, wireless power allocation, and interference management in a mobile cloud computing environment. To analyze the proposed game, we use the backward induction method to capture the sequential dependencies of decisions at the different levels of the game. We performed the theoretical analysis of the game to obtain an equilibrium at each level, and we also present the algorithms for each level and describe the implementation used to achieve the equilibrium states.

In order to make the proposed mobile cloud computing system more convincing, the system model was extended to support multiple telecom operators, macro cells, 
and small cells. The problem was then formulated as a three-level Stackberg Game, and the replicator dynamics method was adopted to solve the evolutionary game between the groups of small cell base stations. At the level of the cloud mobile service providers, we proposed a homogeneous Bertrand game with multiple CMM SPs and used a backward induction method to solve the whole model.

We designed and performed simulations to evaluate the proposed Stackelberg game theoretical method in these two mobile cloud computing systems. The simulation results presented that cloud operations have a significant impact on the performance of heterogeneous wireless network, and thus, the joint optimization of operations of clouds and wireless networks is necessary. This is due to the unique dynamics tied with cloud, CMM services and wireless networks. By jointly optimizing the operations of clouds and wireless networks, the proposed approach can considerably improve the performance of the mobile cloud computing system.

\subsection{Future Work}

In future research, we would like to design a new game theoretic approach for the operations of cloud and wireless networks in mobile cloud computing environment. This new approach should consider wireless network virtualization for enabling the abstraction of network infrastructure and radio spectrum resources. This will significantly reduce the overall expenses of operation of the network by integrating wireless network virtualization with our proposed mobile cloud computing framework. We would also like to extend the proposed game theoretic approach in this dissertation that applies to multiple cooperative small cells which may also suffer interference among them. Last but not least, we would like to devise more accurate and pragmatic utility functions to describe the CMM service price decision, resource allocation, and the interference management in a mobile cloud computing system. 


\section{List of References}

[1] ERICSSON, "The telecom cloud opportunity," in ERICSSON Discussion Paper, (http://www.ericsson.com/, accessed on Sept. 26, 2013.), Mar. 2012.

[2] R. Kokku, R. Mahindra, H. Zhang, and S. Rangarajan, "NVS: a substrate for virtualizing wireless resources in cellular networks," IEEE/ACM Trans. Netw., vol. 20, no. 5, pp. 1333-1346, 2012.

[3] B. Calder and Wang, "Windows azure storage: A highly available cloud storage service with strong consistency," in Proc. 23rd ACM SOSP'2011, (New York, NY, USA), pp. 143-157, ACM, 2011.

[4] D. Kovachev, D. Renzel, R. Klamma, and Y. Cao, "Mobile community cloud computing: emerges and evolves," in Proc. IEEE Eleventh MDM'10, pp. 393395, 2010.

[5] B. Wang, Z. Han, and K. J. R. Liu, "Distributed relay selection and power control for multiuser cooperative communication networks using Stackelberg game," IEEE Trans. Mobile Comput., vol. 8, pp. 975-990, Jul. 2009.

[6] S. Hart, "Evolutionary dynamics and backward induction," Games and Economic Behavior, vol. 41, no. 2, pp. 227-264, 2002.

[7] Z. Sanaei, S. Abolfazli, A. Gani, and M. Shiraz, "Sami: Service-based arbitrated multi-tier infrastructure for mobile cloud computing," in Proc. ICCC'12, pp. 1419, IEEE, 2012.

[8] N. Fernando, S. W. Loke, and W. Rahayu, "Mobile cloud computing: A survey," Elsevier Future Generation Computer Systems, vol. 29, pp. 84-106, Jan. 2013.

[9] D. Huang et al., "Mobile cloud computing," IEEE MMTC E-Letter, vol. 6, no. 10, pp. 27-31, 2011. 
[10] R.-S. Chang, J. Gao, V. Gruhn, J. He, G. Roussos, and W.-T. Tsai, "Mobile cloud computing research - issues, challenges and needs," in IEEE 7th Int'l Symp. Service Oriented System Engineering (SOSE), pp. 442-453, Mar. 2013.

[11] K. Kumar and L. Yung-Hsiang, "Cloud computing for mobile users: Can offloading computation save energy?," Computer, vol. 43, no. 4, pp. 51-56, 2010.

[12] S. Bu, F. R. Yu, and Y. Cai, "When the smart grid meets energy-efficient communications: Green wireless cellular networks powered by the smart grid," IEEE Trans. Wireless Comm., vol. 11, no. 8, pp. 3014-3024, 2012.

[13] H. Ballani, T. Karagiannis, and P. Costa, "Towards predictable datacenter networks," in Proc. ACM SIGCOMM'2011, pp. 242-253, 2011.

[14] P. Costa, M. Migliavacca, P. Pietzuch, and A. L. Wolf, "NaaS: Network-as-aservice in the cloud," in Proc. 2nd USENIX Hot-ICE'12, vol. 12, pp. 1-1, 2012.

[15] Z. Chen and D. Wu, "Rate-distortion optimized cross-layer rate control in wireless video communication," IEEE Trans. Circ. Sys. Video Tech., vol. 22, pp. 352365, Mar. 2012.

[16] H. Balakrishnan, V. N. Padmanabhan, S. Seshan, and R. H. Katz, "A comparison of mechanisms for improving TCP performance over wireless links," IEEE/ACM Trans. Netw., vol. 5, pp. 756-769, Dec. 1997.

[17] J. Huang, Z. Li, M. Chiang, and A. K. Katsaggelos, "Joint source adaptation and resource allocation for multi-user wireless video streaming," IEEE Trans. CSVT, vol. 18, no. 5, pp. 582-595, 2008.

[18] S. Wang and S. Dey, "Rendering adaptation to address communication and computation constraints in cloud mobile gaming," in Proc. IEEE Globecom'2010, pp. 1-6, 2010.

[19] Nokia, "Network virtualization: Enabling novel business models in a dynamic market," tech. rep., Tech. Rep, 2012.

[20] A. Corradi, M. Fanelli, and L. Foschini, "Vm consolidation: a real case based on openstack cloud," Future Generation Computer Systems, vol. 32, pp. 118-127, 2014 .

[21] S. Sezer, S. Scott-Hayward, P. K. Chouhan, B. Fraser, D. Lake, J. Finnegan, N. Viljoen, M. Miller, and N. Rao, "Are we ready for SDN? implementation 
challenges for software-defined networks," IEEE Commun. Mag., vol. 51, Jul. 2013.

[22] ONF, "Onf white paper: Software-defined networking: The new norm for networks," tech. rep., ONF, 2012.

[23] X. Wang, P. Krishnamurthy, and D. Tipper, "Wireless network virtualization," in Proc. ICNC'13, pp. 818-822, IEEE, 2013.

[24] Juniper Research, "Mobile cloud: Smart device strategies for enterprise and consumer markets 2011-2016," tech. rep., Tech. Rep, http://juniperresearch.com, July 2011.

[25] X. Renchao, F. R. Yu, J. Hong, and L. Yi, "Energy-efficient resource allocation for heterogeneous cognitive radio networks with femtocells," IEEE Trans. Wireless Comm, vol. 11, no. 11, pp. 3910-3920, 2012.

[26] A. Ghosh et al., "Heterogeneous cellular networks: From theory to practice," IEEE Comm. Mag., vol. 50, no. 6, pp. 54-64, June 2012.

[27] A. S. Prasad and S. Rao, "A mechanism design approach to resource procurement in cloud computing," IEEE Trans. Computers, vol. 63, no. 1, pp. 17-30, 2014.

[28] J. Von Neumann and O. Morgenstern, "Theory of games and economic behavior," Bull. Amer. Math. Soc, vol. 51, no. 7, pp. 498-504, 1945.

[29] J. Nash, "Non-cooperative games," Annals of mathematics, pp. 286-295, 1951.

[30] A. K. Dixit, S. Skeath, and D. Reiley, Games of strategy. Norton New York, 1999.

[31] B. Wang, Y. Wu, and K. Liu, "Game theory for cognitive radio networks: An overview," Computer Networks, vol. 54, no. 14, pp. 2537-2561, 2010.

[32] B. Wang, Y. Wu, and K. J. R. Liu, "Game theory for cognitive radio networks: An overview," Computer Networks, vol. 54, no. 14, pp. 2537-2561, 2010.

[33] R. Buyya, D. Abramson, J. Giddy, and H. Stockinger, "Economic models for resource management and scheduling in grid computing," Concurrency and Computation: Practice and Experience, vol. 14, no. 13-15, pp. 1507-1542, 2002.

[34] N. Fernando, S. Loke, R. W., and Wenny, "Mobile cloud computing: A survey," Future Generation Computer Systems, vol. 29, no. 1, pp. 84-106, 2013. 
[35] T. Benson, A. Akella, A. Shaikh, and S. Sahu, "Cloudnaas: a cloud networking platform for enterprise applications," in Proc. ACM Sympo. on Cloud Computing, p. 8, ACM, 2011.

[36] Y.-F. R. Chen and R. Jana, "Speedgate: A smart data pricing testbed based on speed tiers," in Proc. IEEE INFOCOM'13, pp. 3195-3200, 2013.

[37] S. Shakkottai and R. Srikant, "Economics of network pricing with multiple ISPs," IEEE/ACM Trans. Networking, vol. 14, no. 6, pp. 1233-1245, 2006.

[38] M. Andrews, U. Ozen, M. I. Reiman, and Q. Wang, "Economic models of sponsored content in wireless networks with uncertain demand," in Proc. IEEE INFOCOM'13, pp. 3213-3218, IEEE, 2013.

[39] P. Marbach, "Analysis of a static pricing scheme for priority services," IEEE/ACM Trans. Networking, vol. 12, no. 2, pp. 312-325, 2004.

[40] Y. Hayel and B. Tuffin, "A mathematical analysis of the cumulus pricing scheme," Computer networks, vol. 47, no. 6, pp. 907-921, 2005.

[41] S. Ha, S. Sen, C. Joe-Wong, Y. Im, and M. Chiang, "Tube: Time-dependent pricing for mobile data," in Proc. ACM SIGCOMM'12, pp. 247-258, ACM, 2012.

[42] S. Sen, C. Joe-Wong, S. Ha, and M. Chiang, "A survey of smart data pricing: Past proposals, current plans, and future trends," ACM Computing Surveys, 2013.

[43] J. Hoadley and P. Maveddat, "Enabling small cell deployment with HetNet," IEEE Trans. Wireless Commun., vol. 19, no. 2, pp. 4-5, April 2012.

[44] 3GPP, "Further advancements for E-UTRA physical layer aspects (release 9)," 3GPP TR36.814, 2010.

[45] A. Greenberg, J. Hamilton, D. A. Maltz, and P. Patel, "The cost of a cloud: research problems in data center networks," ACM SIGCOMM Comput. Commun. Rev., vol. 39, no. 1, pp. 68-73, 2008.

[46] A. Ledvina and R. Sircar, "Oligopoly games under asymmetric costs and an application to energy production," Mathematics and Financial Economics, vol. 6, no. 4, pp. 261-293, 2012. 
[47] H. D. Sherali, A. L. Soyster, and F. H. Murphy, "Stackelberg-Nash-Cournot equilibria: characterizations and computations," Operations Research, vol. 31, no. 2, pp. 253-276, 1983.

[48] W. Yu, Competition and Cooperation in Multi-user Communication Environments. PhD thesis, Stanford university, 2002.

[49] S. T. Chung, S. J. Kim, J. Lee, and J. M. Cioffi, "A game-theoretic approach to power allocation in frequency-selective Gaussian interference channels," in Proc. IEEE Int'l Symp. Inform. Theory, pp. 316-316, 2003.

[50] J. Huang, R. Cendrillon, M. Chiang, and M. Moonen, "Autonomous spectrum balancing (asb) for frequency selective interference channels," in Proc. IEEE Int'l Symp. Inform. Theory, pp. 610-614, 2006.

[51] G. Scutari, D. P. Palomar, and S. Barbarossa, "Asynchronous iterative waterfilling for Gaussian frequency-selective interference channels: a unified framework," in Proc. Information Theory and Applications Workshop, 2007, pp. 349-358, 2007.

[52] C.-Y. Wang and H.-Y. Wei, "Profit maximization in femtocell service with contract design," IEEE Trans. Wireless Comm, vol. 12, no. 5, pp. 1978-1988, 2013.

[53] X. Renchao, F. R. Yu, and J. Hong, "Spectrum sharing and resource allocation for energy-efficient heterogeneous cognitive radio networks with femtocells," in IEEE ICC'12, pp. 1661-1665, 2012.

[54] S. Wang and S. Dey, "Adaptive mobile cloud computing to enable rich mobile multimedia applications," IEEE Trans. Multimedia, vol. 15, no. 4, pp. 870-883, 2013.

[55] H. T. Dinh, C. Lee, D. Niyato, and P. Wang, "A survey of mobile cloud computing: Architecture, applications, and approaches," Wiley Wireless Comm. and Mobile Computing, 2011. doi: 10.1002/wcm.1203.

[56] S. Dey, "Cloud mobile media: Opportunities, challenges, and directions," in Proc. ICNC'12, pp. 929-933, 2012.

[57] S. Wang and S. Dey, "Modeling and characterizing user experience in a cloud server based mobile gaming approach," in Proc. IEEE GLOBECOM'09, pp. 1-7, 2009 . 
[58] Z. Huang, C. Mei, L. E. Li, and T. Woo, "Cloudstream: delivering highquality streaming videos through a cloud-based svc proxy," in Proc. IEEE INFOCOM'11, pp. 201-205, 2011.

[59] M. Sahraei-Ardakani and A. Rahimi-Kian, "A dynamic replicator model of the players bids in an oligopolistic electricity market," Electric Power Systems Research, vol. 79, no. 5, pp. 781-788, 2009.

[60] F. Chapeau-Blondeau and A. Monir, "Numerical evaluation of the lambert w function and application to generation of generalized gaussian noise with exponent 1/2," IEEE Trans. Signal Proc., vol. 50, no. 9, pp. 2160-2165, 2002.

[61] D. M. Topkis, Supermodularity and complementarity. Princeton University Press, 1998. 